\title{
Complexidade em programação não linear
}

\author{
John Lenon Cardoso Gardenghi
}

\author{
TESE APRESENTADA \\ AO \\ Instituto DE MATEMÁticA E EstatísticA \\ DA \\ Universidade DE SÃo PAUlo \\ PARA \\ OBTENÇÃO DO TÍTULO \\ $\mathrm{DE}$ \\ DOUTOR EM CIÊNCIAS
}

Programa: Ciência da Computação

Orientador: Prof. Dr. Ernesto G. Birgin

Durante o desenvolvimento deste trabalho, o autor contou com o auxílio financeiro da Fundação de Amparo à Pesquisa do Estado de São Paulo (FAPESP), processo no 2013/23494-9.

São Paulo, janeiro de 2018 


\section{Complexidade em programação não linear}

Esta versão da tese contém as correções e alterações sugeridas pela Comissão Julgadora durante a defesa da versão original do trabalho, realizada em 09/08/2017. Uma cópia da versão original está disponível no Instituto de Matemática e Estatística da Universidade de São Paulo.

Banca Examinadora

- Prof. Dr. Ernesto G. Birgin (orientador) - IME-USP

- Prof. Dr. Geovani Nunes Grapiglia - UFPR

- Prof.a Dr.a Elizabeth Wegner Karas - UFPR

- Prof. Dr. José Mario Martínez Perez - Unicamp

- Prof.a Dr.a Sandra Augusta Santos - Unicamp 


\section{Agradecimentos}

Ao Prof. Ernesto, pela orientação, disponibilidade e companheirismo durante o desenvolvimento deste trabalho, bem como pela motivador interesse com que conduziu nossas atividades.

Ao Prof. Mario, pelas inestimáveis sugestões dadas ao desenvolvimento deste trabalho, desde sua concepção até o final. Sem dúvidas, ele é um modelo de pesquisador e grande inspiração para mim.

À Profa. Sandra, pela ativa contribuição neste trabalho e durante todo o meu desenvolvimento acadêmico até aqui, desde a minha iniciação científica. Sua dedicação e amor à docência e pesquisa foi meu primeiro contato com o mundo acadêmico, inspirou e motivou minha caminhada até aqui.

Há algo muito importante que aprendi com os professores deste grupo de otimização contínua e métodos computacionais de otimização, que tão bem me receberam: trabalhe com amigos, ou faça de seus colaboradores seus amigos; assim, de qualquer forma, você sempre estará trabalhando com amigos. Neste grupo me senti, e me sinto, trabalhando entre amigos.

Aos meus colegas do laboratório, que me proporcionaram momentos de descontração, bem como de reflexão e motivação. Em especial, aos meus amigos do grupo de otimização contínua, pelas sugestões que colaboraram ao desenvolvimento deste trabalho.

Ao meu pai, que sempre me acompanhou, investiu e acreditou em mim.

À minha querida Aninha, por tudo. Por trazer alegria aos meus dias, por me amar e se deixar amar por mim, por ser minha inspiração para viver dias bons e força para viver dias difíceis. 


\section{Resumo}

GARDENGHI, J. L. Complexidade em programação não linear. 2017. Tese (Doutorado) Instituto de Matemática e Estatística, Universidade de São Paulo, São Paulo, 2017.

No presente trabalho, estudamos e desenvolvemos algoritmos com análise de complexidade de avaliação de pior caso para problemas de programação não linear. Para minimização irrestrita, estabelecemos dois algoritmos semelhantes que exploram modelos de ordem superior com estratégia de regularização. Propusemos uma implementação computacional que preserva as boas propriedades teóricas de complexidade, e fizemos experimentos numéricas com problemas clássicos da literatura, a fim de atestar a implementação e avaliar a aplicabilidade de métodos que empreguem modelos de ordem superior. Para minimização com restrições, estabelecemos um algoritmo de duas fases que converge a pontos que satisfazem condições de otimalidade de primeira ordem não escaladas para o problema de programação não linear.

Palavras-chave: programação não linear, otimização, complexidade de avaliação, experimentos numéricos. 


\section{Abstract}

GARDENGHI, J. L. Complexity in nonlinear programming. 2017. Doctoral Thesis - Institute of Mathematics and Statistics, University of São Paulo, São Paulo, 2017.

In the present work, we have studied and developed algorithms with worst-case evaluation complexity analysis for nonlinear programming problems. For the unconstrained optimization case, we have established two similar algorithms that explore high-order regularization models. We have proposed a computational implementation that preserves the good properties of the evaluation complexity theory, and we made numerical experiments with classical problems from the literature, in order to check the implementation and certify the practical applicability of methods that employ high-order models. For the constrained optimization case, we have established a two phases algorithm that converges to points that meet the unscaled first-order optimality condition for the nonlinear programming problem.

Keywords: nonlinear programming, optimization, evaluation complexity, numerical experiments. 


\section{Sumário}

1 Introdução $\quad 1$

2 Complexidade em otimização sem restrições 5

2.1 Um algoritmo de descida de primeira ordem . . . . . . . . . . . . . . . 5

2.2 Algoritmos com modelos de ordem superior . . . . . . . . . . . . . . . . . . . 9

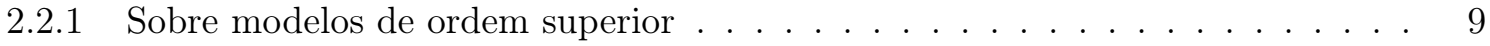

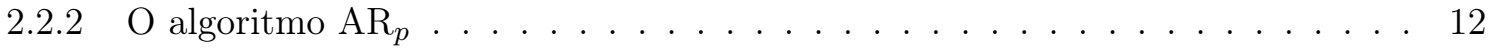

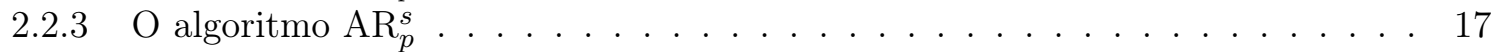

2.2.4 Considerações sobre as duas abordagens . . . . . . . . . . . . . 20

3 Complexidade em otimização com restrições 23

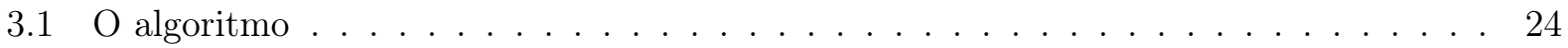

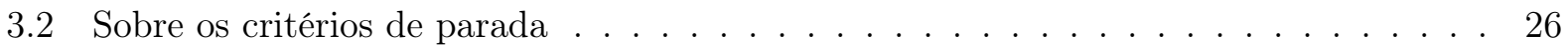

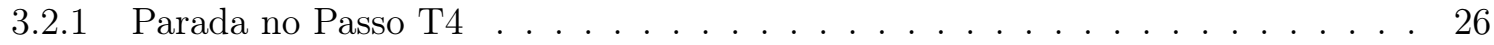

3.2 .2 Parada no Passo F2 ou no Passo T3 . . . . . . . . . . . . . . . . . 27

3.3 Boa definição, convergência e complexidade . . . . . . . . . . . . . . 30

3.4 Considerações sobre os resultados de complexidade . . . . . . . . . . . . . . . 37

4 Implementação e experimentos numéricos $\quad 41$

4.1 Implementação computacional: $\mathrm{AR}_{3} \ldots \ldots \ldots \ldots \ldots \ldots \ldots$. . . . . . . . . 41

4.1.1 Um algoritmo para minimização de polinômios quárticos normais . . . . . . . 43

4.1.2 Gloptipoly: um método para minimização global de polinômios . . . . . . . . 53

4.2 Experimentos com Gloptipoly e GMNQP . . . . . . . . . . . . . . . . . . . 59

4.3 Experimentos numéricos com $\mathrm{AR}_{3} \ldots \ldots \ldots \ldots \ldots \ldots \ldots$

4.4 Implementação computacional: $\mathrm{AR}_{2}^{s}$ e $\mathrm{AR}_{3}^{s} \ldots \ldots \ldots \ldots \ldots$. . . . . . . . . 62

4.4 .1 Subproblemas . . . . . . . . . . . . . . . . . . . . 62

4.4.2 Sobre as condições de descida e aceitação do ponto candidato . . . . . . . . . 63

4.4.3 Norma do termo de regularização do modelo . . . . . . . . . . . . . . . . . . . 64

4.4.4 Implicações do uso de controle de passo . . . . . . . . . . . . . . . . . 65

4.4.5 Critérios de parada . . . . . . . . . . . . . . . 65

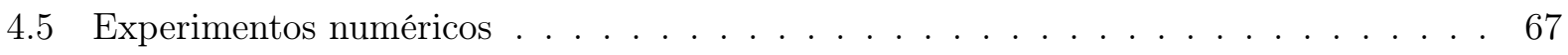

4.5.1 Experimentos com problemas clássicos da literatura . . . . . . . . . . 67

$4.5 .2 \quad$ Um problema de grande porte . . . . . . . . . . . . . . . . 73

$\begin{array}{lll}5 & \text { Considerações finais e trabalhos futuros } & 77\end{array}$

$\begin{array}{ll}\text { Referências Bibliográficas } & 79\end{array}$ 


\section{Capítulo 1}

\section{Introdução}

Complexidade de algoritmos é um assunto bem difundido e conhecido na área de análise de algoritmos em Ciência da Computação. Em tais estudos, o objetivo é estabelecer o custo computacional, em termos de alguma medida de tamanho da entrada, que um dado algoritmo consome para resolver um determinado problema.

Recentemente, há um interesse crescente no estudo de complexidade de algoritmos para problemas de programação não linear e não convexa. Estudos neste contexto começaram com o trabalho de Nemirovsky e Yudin [57].

Complexidade em programação não linear está associada a uma tripla $\left(\Sigma, \mathcal{O}, \tau_{\varepsilon}\right)$ [39], de forma que

- $\Sigma$ é uma classe de problemas (minimização convexa, quadrados mínimos, minimização irrestrita...)

- $\mathcal{O}$ é um oráculo que fornece informações sobre a função objetivo, e suas derivadas, num dado ponto $x$.

- $\tau_{\varepsilon}$ é um critério de parada, associado a uma precisão $\varepsilon$.

Neste contexto, algoritmos para programação não linear, cuja complexidade desejamos avaliar, objetivam encontrar um ponto que satisfaça $\tau_{\varepsilon}$, para problemas da classe $\Sigma$, usando apenas informações do oráculo $\mathcal{O}$. Desta forma, deseja-se estudar

- A quantidade de iterações, isto é, contar quantas iterações, ou chamadas ao oráculo $\mathcal{O}$, são necessárias para resolver algum problema da classe $\Sigma$. Esse valor dependerá principalmente de $\varepsilon$ e de parâmetros associados ao problema e ao algoritmo em questão.

- O desempenho numérico, que é um limitante nas operações aritméticas necessárias no pior caso, para resolver o problema em questão. Esse desempenho é um valor que costuma ser proporcional à quantidade de iterações.

- A complexidade do esquema $\left(\Sigma, \mathcal{O}, \tau_{\varepsilon}\right)$ é o desempenho (numérico ou quantidade de iterações) do melhor algoritmo possível para o esquema.

No presente trabalho, estamos interessados em estudar a complexidade de algoritmos específicos, e não para esquema $\left(\Sigma, \mathcal{O}, \tau_{\varepsilon}\right)$ inteiro. Em outras palavras, queremos contar quantas iterações (ou 
quantas avaliações de função, ou alguma outra medida quantitativa) são necessárias para que um método convirja a um ponto com determinadas propriedades para um dado problema.

Ao longo dos últimos anos, métodos clássicos de otimização tiveram sua complexidade estudada, bem como novos algoritmos para diversos problemas de otimização foram propostos, com suas respectivas complexidades avaliadas.

Em se tratando de problemas de minimização sem restrições, Nesterov [58, p. 29] demonstra que o método de máxima descida com Goldstein-Armijo converge com complexidade $\mathcal{O}\left(1 / \epsilon^{2}\right)$ a um ponto $x^{\epsilon}$ que satisfaz $\left\|\nabla f\left(x^{\epsilon}\right)\right\| \leq \epsilon$. Este é um dos pioneiros que introduziram a ideia de complexidade de avaliação, e a partir do qual o assunto tornou-se um tema ativo de pesquisa em programação não linear.

Nesterov e Polyak [59] e Cartis, Gould e Toint [21,22,24] apresentam um método de Newton com regularização cúbica para minimização irrestrita que consome tempo computacional $\mathcal{O}\left(1 / \epsilon^{3 / 2}\right)$ para atingir um ponto cuja norma do gradiente seja inferior a $\epsilon$.

Cartis, Gould e Toint [20] mostram que a análise de pior caso de Nesterov para o método de máxima descida é justa, e que o mesmo acontece para o método Newton tradicional. Ademais, os autores demonstram que existe um problema que consome o tempo $\mathcal{O}\left(1 / \epsilon^{3 / 2}\right)$ que Nesterov e Polyak [59] e Cartis, Gould e Toint [21,22,24] mostraram ser necessário para o método de Newton com regularização cúbica.

Cartis, Gould e Toint [23] apresentam métodos de primeira ordem para o problema de minimização de uma função composta

$$
\Phi_{h}(x)=f(x)+h(c(x)),
$$

onde $h: \mathbb{R}^{m} \rightarrow \mathbb{R}$ é convexa mas não suave, $f: \mathbb{R}^{n} \rightarrow \mathbb{R}$ e $c: \mathbb{R}^{n} \rightarrow \mathbb{R}^{m}$ funções continuamente diferenciáveis no domínio de interesse, todavia potencialmente não convexas. Neste trabalho, os autores demonstram complexidade de $\mathcal{O}\left(1 / \epsilon^{2}\right)$ para o problema proposto.

Por outro lado, Cartis, Gould e Toint $[27,28]$ propõem um método de primeira ordem para a resolução do problema de minimização com restrições

$$
\begin{aligned}
\text { Minimizar } & f(x) \\
\text { s. a } & h(x)=0 \\
& g(x) \leq 0,
\end{aligned}
$$

sendo $f, g$ e $h$ funções continuamente diferenciáveis do $\mathbb{R}^{n}$ em $\mathbb{R}, \mathbb{R}^{m}$ e $\mathbb{R}^{q}$, respectivamente. Este método converge com complexidade $\mathcal{O}\left(1 / \epsilon^{2}\right)$ a um ponto $x^{\epsilon}$ que satisfaz

$$
\begin{aligned}
\left\|\left(\begin{array}{c}
h\left(x^{\epsilon}\right) \\
\max \left\{0, g\left(x^{\epsilon}\right)\right\}
\end{array}\right)\right\| & \leq \eta \\
\max \left\{\frac{\left\|\nabla f\left(x^{\epsilon}\right)+\nabla h\left(x^{\epsilon}\right) \lambda^{\epsilon}+\nabla g\left(x^{\epsilon}\right) \mu^{\epsilon}\right\|}{\max \left\{1,\left\|\lambda^{\epsilon}\right\|_{D},\left\|\mu^{\epsilon}\right\|_{D}\right\}}, \frac{g\left(x^{\epsilon}\right)^{T} \mu^{\epsilon}}{\max \left\{1,\left\|\mu^{\epsilon}\right\|_{D}\right\}}\right\} & \leq \epsilon \\
\mu_{i}^{\epsilon} & \geq 0, \text { para } i=1, \ldots, q,
\end{aligned}
$$

onde $\lambda^{\epsilon} \in \mathbb{R}^{m}, \mu^{\epsilon} \in \mathbb{R}^{q}$ e $\nabla h(x)$ e $\nabla g(x)$ são as transpostas do Jacobiano de $h$ e $g$, respectivamente, e $\|\cdot\|_{D}$ é a norma dual, induzida pelo produto interno, da norma $\|\cdot\|$ escolhida. Por se tratar de um método de primeira ordem e dado que Cartis, Gould e Toint [20] mostraram que a complexidade $\mathcal{O}\left(1 / \epsilon^{2}\right)$ é justa, muito provavelmente não se obtenha métodos de primeira ordem para a resolução do problema de minimização com restrições que possuam menor complexidade. É notável que a presença de restrições, potencialmente não lineares e não convexas, não afetem a complexidade de métodos deste tipo. Não obstante, o método apresentado não possui cunho prático. Isto significa 
que, apesar de possuir boas propriedades teóricas, o algoritmo proposto não possui um desempenho satisfatório na prática.

Atualmente, há uma intensa atividade de pesquisa em complexidade de avaliação em programação não linear. Entre os muitos trabalhos que vem sendo publicados, podemos citar, por exemplo, $[5,6,15,16,19,29-31,33-37,40-45,52,53,55]$. Por conseguinte, complexidade em programação não linear é um tema de pesquisa ativo e relevante para a área.

O objetivo da presente tese é introduzir três algoritmos, e se organiza da seguinte forma. No Capítulo 2, introduziremos dois algoritmos, bem semelhantes, para problemas de minimização sem restrições, que usam modelos de ordem superior com estratégia de regularização. No Capítulo 3, introduziremos um algoritmo para minimização com restrições, que é o primeiro algoritmo com convergência a pontos KKT não escalados a possuir demonstração de complexidade de avaliação. No Capítulo 4, realizaremos experimentos numéricos com implementações dos algoritmos propostos para minimização sem restrições. 


\section{Capítulo 2}

\section{Complexidade em otimização sem restrições}

Neste capítulo, estamos interessados em estudar a complexidade de avaliação de pior caso de métodos para o problema

$$
\underset{x \in \mathbb{R}^{n}}{\operatorname{Minimizar}} f(x),
$$

onde $f: \mathbb{R}^{n} \rightarrow \mathbb{R}$ (a) é uma função $p$ vezes continuamente diferenciável, $p \geq 1$, com derivada de ordem $p$ Lipschitz contínua, ou (b) é uma função $p+1$ vezes continuamente diferenciável.

Este capítulo organiza-se da seguinte forma. Na Seção 2.1, analisaremos a complexidade de avaliação de um algoritmo clássico para problemas de minimização sem restrições. Na Seção 2.2, introduziremos dois algoritmos para minimização sem restrições que empregam modelos de ordem superior e estratégia de regularização e estabeleceremos suas respectivas complexidades de avaliação.

\subsection{Um algoritmo de descida de primeira ordem}

Algoritmos de descida de primeira ordem são clássicos para problemas de minimização irrestrita. Nesta seção, estudaremos a complexidade de um algoritmo de descida de primeira ordem clássico, com condição de decréscimo suficiente de Armijo. Antes de tudo, começaremos descrevendo o método no Algoritmo 2.1. Com isso, temos o objetivo de ter um primeiro contato com a técnica análise de complexidade de avaliação, estabelecendo uma demonstração simples e clara para este algoritmo, clássico para problemas de minimização sem restrições.

\section{Algoritmo 2.1: Método de Descida de Primeira Ordem}

Entrada. $\epsilon>0, \bar{\beta} \geq \underline{\beta}>0, \gamma \in\left(0, \frac{1}{2}\right), \theta \in(0,1), x^{0} \in \mathbb{R}^{n}$. Inicialize $k \leftarrow 0$.

Passo 1. Se $\left\|\nabla f\left(x^{k}\right)\right\| \leq \epsilon$, então pare.

Passo 2. Calcule $d^{k} \in \mathbb{R}^{n}$ tal que

$$
\begin{aligned}
& \nabla f\left(x^{k}\right)^{T} d^{k} \leq-\theta\left\|\nabla f\left(x^{k}\right)\right\|\left\|d^{k}\right\| \mathrm{e} \\
& \underline{\beta}\left\|\nabla f\left(x^{k}\right)\right\| \leq\left\|d^{k}\right\| \leq \bar{\beta}\left\|\nabla f\left(x^{k}\right)\right\| .
\end{aligned}
$$


Passo 3. Calcule $\alpha_{k}$ como sendo o maior elemento da sequência $\left\{1, \frac{1}{2}, \frac{1}{4}, \frac{1}{8}, \ldots\right\}$ tal que

$$
f\left(x^{k}+\alpha_{k} d^{k}\right) \leq f\left(x^{k}\right)+\gamma \alpha_{k} \nabla f\left(x^{k}\right)^{T} d^{k} .
$$

Passo 4. Calcule $x^{k+1}=x^{k}+\alpha_{k} d^{k}$, faça $k \leftarrow k+1$ e vá para o Passo 1 .

Para demonstrar sua complexidade, assumiremos algumas hipóteses.

Hipótese 2.1. $f \in \mathcal{C}^{1}$ e $f(x) \geq f_{\min }$, para todo $x \in \mathbb{R}^{n}$.

Hipótese 2.2. Existe $L>0$ tal que $\|\nabla f(y)-\nabla f(x)\| \leq L\|y-x\|$, para todo $x, y \in \mathbb{R}^{n}$.

Inicialmente, enunciaremos um resultado que estabelece uma consequência importante da Hipótese 2.2. Para isso, usaremos o seguinte teorema.

Teorema 2.1. (Fórmula de Taylor com resto integral) Se $f: \mathbb{R}^{n} \rightarrow \mathbb{R}$ é continuamente diferenciável no intervalo de extremos $x$ e $x+d$ pertencentes a $\mathbb{R}^{n}$, então definimos a aproximação de Taylor com resto integral de ordem um para $f$ em torno de $x$ como

$$
f(x+d)=f(x)+\int_{0}^{1} \nabla f(x+\xi d)^{T} d d \xi
$$

Lema 2.2. Se a Hipótese 2.2 vale, então

$$
f(x+d) \leq f(x)+\nabla f(x)^{T} d+\frac{L}{2}\|d\|^{2},
$$

para todo $x, d \in \mathbb{R}^{n}$.

Demonstração. Tomando $y=x+d$, da Hipótese 2.2 segue que

$$
\|\nabla f(x+d)-\nabla f(x)\| \leq L\|d\|,
$$


para todo $x, d \in \mathbb{R}^{n}$. De (2.5), da desigualdade de Cauchy-Schwarz e de (2.7), segue que

$$
\begin{aligned}
f(x+d) & =f(x)+\int_{0}^{1} \nabla f(x+\xi d)^{T} d d \xi \\
& =f(x)+\nabla f(x)^{T} d-\nabla f(x)^{T} d+\int_{0}^{1} \nabla f(x+\xi d)^{T} d d \xi \\
& =f(x)+\nabla f(x)^{T} d+\int_{0}^{1}[\nabla f(x+\xi d)-\nabla f(x)]^{T} d d \xi \\
& \leq f(x)+\nabla f(x)^{T} d+\int_{0}^{1}\|\nabla f(x+\xi d)-\nabla f(x)\|\|d\| d \xi \\
& \leq f(x)+\nabla f(x)^{T} d+\int_{0}^{1} L\|\xi d\|\|d\| d \xi \\
& =f(x)+\nabla f(x)^{T} d+L\|d\|^{2} \int_{0}^{1} \xi d \xi \\
& =f(x)+\nabla f(x)^{T} d+\frac{L}{2}\|d\|^{2},
\end{aligned}
$$

como queríamos demonstrar.

O teorema a seguir estabelece a complexidade do Algoritmo 2.1.

Teorema 2.3. Suponhamos que valham as Hipóteses 2.1 e 2.2, que $\epsilon>0$ e que o Algoritmo 2.1 para no Passo 1 quando $\left\|\nabla f\left(x^{k}\right)\right\| \leq \epsilon$. Então, o Algoritmo 2.1 consome, no máximo,

$$
\left\lfloor\kappa\left[\frac{f\left(x^{0}\right)-f_{\min }}{\epsilon^{2}}\right]\right\rfloor
$$

iterações, onde

$$
\kappa=\left(\gamma \theta \underline{\beta} \min \left\{1, \frac{(1-\gamma) \theta}{\bar{\beta} L}\right\}\right)^{-1}
$$

Demonstração. Tomemos $\alpha>0$ tal que

$$
\alpha \leq \frac{2(1-\gamma) \theta}{\bar{\beta} L}=\kappa_{\alpha}
$$

Por (2.3), temos que $\frac{1}{\bar{\beta}} \leq \frac{\left\|\nabla f\left(x^{k}\right)\right\|}{\left\|d^{k}\right\|}$. Assim sendo, de (2.9) vem que

$$
\alpha \leq \frac{2(1-\gamma) \theta}{L} \frac{\left\|\nabla f\left(x^{k}\right)\right\|}{\left\|d^{k}\right\|} .
$$

Por outro lado, de (2.2) temos que, se $\left\|\nabla f\left(x^{k}\right)\right\| \neq 0$, então $\theta \leq-\frac{\nabla f\left(x^{k}\right)^{T} d^{k}}{\left\|\nabla f\left(x^{k}\right)\right\|\left\|d^{k}\right\|}$. Com isso, de (2.10) segue que

$$
\alpha \leq \frac{2(1-\gamma)}{L} \frac{\left[-\nabla f\left(x^{k}\right)^{T} d^{k}\right]}{\left\|\nabla f\left(x^{k}\right)\right\|\left\|d^{k}\right\|} \frac{\left\|\nabla f\left(x^{k}\right)\right\|}{\left\|d^{k}\right\|}=\frac{2(\gamma-1)}{L} \frac{\nabla f\left(x^{k}\right)^{T} d^{k}}{\left\|d^{k}\right\|^{2}} .
$$

Por conseguinte,

$$
\nabla f\left(x^{k}\right)^{T} d^{k}+\frac{L}{2} \alpha\left\|d^{k}\right\|^{2} \leq \gamma \nabla f\left(x^{k}\right)^{T} d^{k}
$$


Multiplicando ambos os lados de (2.11) por $\alpha$ e somando $f\left(x^{k}\right)$, vem que

$$
f\left(x^{k}\right)+\alpha \nabla f\left(x^{k}\right)^{T} d^{k}+\frac{L}{2} \alpha^{2}\left\|d^{k}\right\|^{2} \leq f\left(x^{k}\right)+\gamma \alpha \nabla f\left(x^{k}\right)^{T} d^{k} .
$$

Consequentemente, por (2.6),

$$
f\left(x^{k}+\alpha d^{k}\right) \leq f\left(x^{k}\right)+\gamma \alpha \nabla f\left(x^{k}\right)^{T} d^{k} .
$$

Como existe $\alpha>0$, limitado superiormente por uma constante $\kappa_{\alpha}$ definida em (2.9), que satisfaz Armijo (2.12), então o mecanismo de backtracking do Passo 3 do Algoritmo 2.1 garante que

$$
\alpha_{k} \geq \min \left\{1, \frac{\kappa_{\alpha}}{2}\right\}=\min \left\{1, \frac{(1-\gamma) \theta}{\bar{\beta} L}\right\}
$$

Isso implica que a quantidade de backtrackings necessária para obter decréscimo suficiente (2.4) é limitada por uma constante que depende somente de $\gamma, \theta, \beta$ e $L$. Além disso, quando a condição de decréscimo suficiente (2.4) é satisfeita, temos que

$$
\begin{aligned}
f\left(x^{k+1}\right) & \leq f\left(x^{k}\right)+\gamma \alpha_{k} \nabla f\left(x^{k}\right)^{T} d^{k} \\
& \leq f\left(x^{k}\right)-\gamma \alpha_{k} \theta\left\|\nabla f\left(x^{k}\right)\right\|\left\|d^{k}\right\|, \text { por }(2.2) \\
& \leq f\left(x^{k}\right)-\gamma \alpha_{k} \theta \underline{\beta}\left\|\nabla f\left(x^{k}\right)\right\|^{2}, \text { por }(2.3) \\
& \leq f\left(x^{k}\right)-\gamma \min \left\{1, \frac{(1-\gamma) \theta}{\bar{\beta} L}\right\} \theta \underline{\beta}\left\|\nabla f\left(x^{k}\right)\right\|^{2}, \text { por }(2.13) .
\end{aligned}
$$

Assim sendo, se $\left\|\nabla f\left(x^{k}\right)\right\|>\epsilon$,

$$
f\left(x^{k+1}\right)<f\left(x^{k}\right)-\gamma \min \left\{1, \frac{(1-\gamma) \theta}{\bar{\beta} L}\right\} \theta \underline{\beta} \epsilon^{2} .
$$

A desigualdade (2.14), junto com a Hipótese 2.1, implicam que a quantidade de iterações em que $\left\|\nabla f\left(x^{k}\right)\right\|>\epsilon$ não excede

$$
\left\lfloor\frac{f\left(x^{0}\right)-f_{\min }}{\gamma \theta \underline{\beta} \min \left\{1, \frac{(1-\gamma) \theta}{\bar{\beta} L}\right\} \epsilon^{2}}\right\rfloor .
$$

Portanto, se o Algoritmo 2.1 para no Passo 1 quando $\left\|\nabla f\left(x^{k}\right)\right\| \leq \epsilon$, então a quantidade de iterações despendidas pelo algoritmo será, no máximo,

$$
\left\lfloor\kappa\left[\frac{f\left(x^{0}\right)-f_{\min }}{\epsilon^{2}}\right]\right\rfloor,
$$

onde $\kappa$, definida em (2.8), é uma constante que depende apenas de $\gamma, \theta, \underline{\beta}, \bar{\beta}$ e $L$. Segue o resultado.

Cabe aqui um breve comentário sobre o Algoritmo 2.1. Na literatura de programação não linear irrestrita, as direções de busca calculadas em métodos de descida clássicos de primeira ordem devem cumprir duas condições [14,54]: a chamada condição do ângulo, que é precisamente $(2.2)$ e cujo objetivo consiste em evitar direções quase perpendiculares ao gradiente, e a condição da norma,

$$
\left\|d^{k}\right\| \geq \beta\left\|\nabla f\left(x^{k}\right)\right\|
$$


também chamada de "condição $\beta$ ", para algum $\beta>0$, cujo objetivo é impedir direções arbitrariamente pequenas durante a execução do algoritmo. Neste caso, impomos no Algoritmo 2.1 uma condição similar, (2.3), que, além de estabelecer um limitante inferior à norma da direção na iteração $k$, também determina uma cota superior. Tal condição é naturalmente satisfeita em métodos de segunda ordem, como o método de Newton, quando a Hessiana, ou sua aproximação, possui norma limitada. Essa condição se faz aqui necessária pois seríamos incapazes de limitar a quantidade de iterações empregadas pelo Algoritmo 2.1 caso a direção crescesse indefinidamente conforme $k$ tendesse a infinito.

\subsection{Algoritmos com modelos de ordem superior}

Nesta seção, apresentaremos dois algoritmos baseados em modelos de ordem superior. $\mathrm{O} \mathrm{AR}_{p}$ (Adaptive Regularization of order $p$ ) é um algoritmo com regularização de ordem $p+1$ apresentado em [10]. É inspirado no método de regularização cúbica [21,22,24,59], baseado na generalização destes resultados usando modelos de ordem superior. Com uma pequena modificação, propomos aqui o $\mathrm{AR}_{p}^{s}$ (Adaptive Regularization of order $p$ Simplified), que, ao contrário do $\mathrm{AR}_{p}$, admite parâmetro de regularização nulo e, portanto, favorece intrinsecamente o emprego do passo de Newton. Apresentaremos resultados de complexidade para ambos e ainda discutiremos, ao final, suas diferenças e, no Capítulo 4, discorreremos acerca de sua implementação computacional.

\subsubsection{Sobre modelos de ordem superior}

Seja $T_{p}(x, d)$ o polinômio de Taylor de ordem $p$ de $f$ em $d$, ou seja,

$$
T_{p}(x, d)=f(x)+\nabla f(x) d+\frac{1}{2} \nabla^{2} f(x) d^{2}+\ldots+\frac{1}{p !} \nabla^{p} f(x) d^{p},
$$

sendo $\nabla^{p} f(x)$, a $p$-ésima derivada de $f$, um tensor de ordem $p$ no $\mathbb{R}^{n}$ e

$$
\nabla^{p} f(x) d^{p}=\sum_{i_{1}, i_{2}, \ldots, i_{p}=1}^{n}\left[\nabla^{p} f(x)\right]_{i_{1} i_{2} \ldots i_{p}} d_{i_{1}} d_{i_{2}} \ldots d_{i_{p}}
$$

a notação tensorial para $\nabla^{p} f(x)$ aplicado $p$ vezes a $d$. Consideremos a seguinte hipótese.

Hipótese 2.3. Dizemos que $x$ e d satisfazem a presente hipótese com escalares $\xi_{1}$ e $\xi_{2}$ positivos se

$$
f(x+d)-T_{p}(x, d) \leq \xi_{1}\|d\|^{p+1}
$$

$e$

$$
\left\|\nabla f(x+d)-\nabla_{d} T_{p}(x, d)\right\| \leq \xi_{2}\|d\|^{p}
$$

Consideremos o modelo de $f$ em torno de $x$ dado por

$$
m_{p}(x, d, \sigma) \stackrel{\text { def }}{=} T_{p}(x, d)+\frac{\sigma}{p+1}\|d\|^{p+1},
$$

onde $\sigma$ é denominado parâmetro de regularização. Notemos que, se o quociente $\sigma /(p+1)$ aproximar o escalar $\xi_{1}$ da Hipótese 2.3, escolher $d$ tal que

$$
m_{p}(x, d, \sigma) \leq m_{p}(x, 0, \sigma),
$$


implica que

$$
T_{p}(x, d)+\xi_{1}\|d\|^{p+1} \leq f(x),
$$

já que $m_{p}(x, 0, \sigma)=f(x)$. Disto, junto com (2.16) segue que

$$
f(x+d) \leq T_{p}(x, d)+\xi_{1}\|d\|^{p+1} \leq f(x) .
$$

Isto sugere um método que, tomando sucessivas direções $d$ que satisfazem (2.19), cause sucessivos decréscimos em $f$ e, por conseguinte, convirja a um minimizador local do problema (2.1).

As questões que surgem naturalmente são como cumprir a Hipótese 2.3 e como obter um $\sigma$ tal que o quociente $\sigma /(p+1)$ aproxime $\xi_{1}$. A primeira questão é mais simples, e responderemos nesta seção. A segunda questão é a inspiração para os métodos que apresentaremos nas Seções 2.2.2 e 2.2.3.

A Hipótese 2.3 cumpre-se em dois cenários: (i) quando $f$ é $p$ vezes continuamente diferenciável e sua derivada de ordem $p$ é Lipschitz contínua ou (ii) quando $f$ é $p+1$ vezes continuamente diferenciável no $\mathbb{R}^{n}$ e sua derivada de ordem $p+1$ é limitada.

Para detalhar melhor as condições de cumprimento da Hipótese 2.3, consideremos o caso em que $f$ é $p$ vezes continuamente diferenciável e sua derivada de ordem $p$ é Lipschitz contínua. Começaremos estendendo o Teorema 2.1 para o caso geral.

Teorema 2.4. (Fórmula de Taylor com resto integral) Se $f: \mathbb{R}^{n} \rightarrow \mathbb{R}$ possui a p-ésima derivada contínua no $\mathbb{R}^{n}$, então definimos a aproximação de Taylor com resto integral de ordem $p$ para $f$ em torno de $x$ como

$$
f(x+d)=T_{p-1}(x, d)+\frac{1}{(p-1) !} \int_{0}^{1}(1-\xi)^{p-1} \nabla^{p} f(x+\xi d) d^{p} d \xi
$$

onde $T_{p-1}(x, d)$ é o polinômio de Taylor de ordem $p-1$ de $f$ em d.

Manipulando (2.20) e sabendo que

$$
\int_{0}^{1}(1-\xi)^{p-1} d \xi=\frac{1}{p}
$$

temos que

$$
\begin{aligned}
f(x+d) & =T_{p-1}(x, d)+\frac{1}{p !} \nabla^{p} f(x) d^{p}-\frac{1}{p !} \nabla^{p} f(x) d^{p}+\frac{1}{(p-1) !} \int_{0}^{1}(1-\xi)^{p-1} \nabla^{p} f(x+\xi d) d^{p} d \xi \\
& =T_{p}(x, d)+\frac{1}{(p-1) !}\left[\int_{0}^{1}(1-\xi)^{p-1} \nabla^{p} f(x+\xi d) d^{p} d \xi-\frac{1}{p} \nabla^{p} f(x) d^{p}\right] \\
& \leq T_{p}(x, d)+\frac{1}{(p-1) !}\left|\int_{0}^{1}(1-\xi)^{p-1}\left[\nabla^{p} f(x+\xi d)-\nabla^{p} f(x)\right] d^{p} d \xi\right| \\
& \leq T_{p}(x, d)+\frac{1}{(p-1) !} \int_{0}^{1}(1-\xi)^{p-1}\left|\left[\nabla^{p} f(x+\xi d)-\nabla^{p} f(x)\right] d^{p}\right| d \xi \\
& \leq T_{p}(x, d)+\frac{1}{(p-1) !}\left[\int_{0}^{1}(1-\xi)^{p-1} d \xi\right] \max _{\xi \in[0,1]}\left|\left[\nabla^{p} f(x+\xi d)-\nabla^{p} f(x)\right] d^{p}\right|
\end{aligned}
$$




$$
\leq T_{p}(x, d)+\frac{1}{p !}\|d\|^{p} \max _{\xi \in[0,1]}\left\|\nabla^{p} f(x+\xi d)-\nabla^{p} f(x)\right\|_{T}
$$

onde $\|\cdot\|_{T}$ é a norma tensorial recursivamente induzida pela norma Euclidiana $\|\cdot\|$ no espaço dos tensores de ordem $p$, dada por

$$
\|M\|_{T}=\max _{\left\|v_{1}\right\|=\ldots=\left\|v_{p}\right\|=1}\left|M\left[v_{1}, \ldots, v_{p}\right]\right|,
$$

sendo $M\left[v_{1}, \ldots, v_{j}\right]$ o tensor de ordem $p-j \geq 0$ resultante da aplicação de $M$ sobre os vetores $v_{1}, \ldots, v_{j}$. Para prosseguir, precisamos da hipótese de que a $p$-ésima derivada de $f$ é Lipschitz contínua. Vamos enunciá-la a seguir.

Hipótese 2.4. Existe uma constante $L \geq 0$ tal que

$$
\left\|\nabla^{p} f(x)-\nabla^{p} f(y)\right\|_{T} \leq(p-1) ! L\|x-y\|
$$

para todo $x, y$ em $\mathbb{R}^{n}$.

De (2.22), pela Hipótese 2.4, temos que

$$
\begin{aligned}
f(x+d) & \leq T_{p}(x, d)+\frac{1}{p !}\|d\|^{p} \max _{\xi \in[0,1]}\{(p-1) ! L \xi\|d\|\} \\
& =T_{p}(x, d)+\frac{L}{p}\|d\|^{p+1} .
\end{aligned}
$$

Deste modo, a condição (2.16) da Hipótese 2.3 cumpre-se para qualquer $x$ e $d \operatorname{com} \xi_{1}=L / p$.

Não obstante, seguindo as ideias de Cartis, Gould e Toint [31], consideremos, para um vetor unitário qualquer $v, \phi(a)=\nabla f(x+a d) v$ and $\tau_{p-1}(a)=\sum_{i=0}^{p-1} \phi^{(i)}(0) a^{i} / i$ !. Por (2.15) e pelo Teorema 2.4, temos que

$$
\phi(1)-\tau_{p-1}(1)=\frac{1}{(p-2) !} \int_{0}(1-\xi)^{p-2}\left[\phi^{(p-1)}(\xi)-\phi^{(p-1)}(0)\right] d \xi .
$$

Por conseguinte, como $\tau_{p-1}(1)=\nabla_{d} T_{p}(x, d) v$,

$$
\left(\nabla f(x+d)-\nabla_{d} T_{p}(x, d)\right) v=\frac{1}{(p-2) !} \int_{0}(1-\xi)^{p-2}\left[\nabla^{p} f(x+\xi s)-\nabla^{p} f(x)\right] d^{p-1} v d \xi
$$

Portanto, explorando a simetria das derivadas de $f$, tomando $v$ para maximizar o valor absoluto do lado esquerdo da inequação (2.25) e considerando (2.21), (2.23) e a Hipótese 2.4, temos que

$$
\begin{aligned}
\| \nabla & f(x+s)-\nabla_{s} T_{p}(x, s) \| \\
& =\frac{1}{(p-2) !}\left|\int_{0}(1-\xi)^{p-2}\left(\nabla^{p} f(x+\xi s)-\nabla^{p} f(x)\right) v\left[\frac{s}{\|s\|}\right]^{p-1}\|s\|^{p-1} d \xi\right| \\
& \leq \frac{1}{(p-2) !}\left[\int_{0}(1-\xi)^{p-2} d \xi\right] \max _{\xi \in[0,1]}\left|\left(\nabla^{p} f(x+\xi s)-\nabla^{p} f(x)\right) v\left[\frac{s}{\|s\|}\right]^{p-1}\right|\|s\|^{p-1} \\
& \leq \frac{1}{(p-1) !} \max _{\xi \in[0,1]\left\|w_{1}\right\|=\cdots=\left\|w_{p}\right\|=1} \mid\left(\nabla^{p} f(x+\xi s)-\nabla^{p} f(x)\right)\left[w_{1}, \ldots, w_{p}\right]\|\| s \|^{p-1} \\
& =\frac{1}{(p-1) !} \max _{\xi \in[0,1]}\left\|\nabla^{p} f(x+\xi s)-\nabla^{p} f(x)\right\|_{T}\|s\|^{p-1} \\
& \leq L\|s\|^{p} .
\end{aligned}
$$


Por conseguinte, a condição (2.17) da Hipótese 2.3 vale para qualquer $x$ e $d$ com $\xi_{2}=L$. Logo, no caso em que $f$ é $p$ vezes continuamente diferenciável com $p$-ésima derivada Lipschitz contínua, vale a Hipótese 2.3.

Consideremos, agora, o caso em que $f$ é $p+1$ vezes continuamente diferenciável e sua derivada de ordem $p+1$ é limitada. Deste último fato, temos que

$$
\left\|\nabla^{p+1} f(x)\right\|_{T} \leq M
$$

para algum $M>0$ e para todo $x \in \mathbb{R}^{n}$. Desta forma, seguindo uma expansão similar à de $(2.22)$,

$$
\begin{aligned}
f(x+d) & =T_{p}(x, d)+\frac{1}{p !} \int_{0}^{1}(1-\xi)^{p} \nabla^{p+1} f(x+\xi d) d^{p+1} d \xi \\
& \leq T_{p}(x, d)+\frac{1}{p !}\left|\int_{0}^{1}(1-\xi)^{p} \nabla^{p+1} f(x+\xi d) d^{p+1} d \xi\right| \\
& \leq T_{p}(x, d)+\frac{1}{p !} \int_{0}^{1}(1-\xi)^{p}\left|\nabla^{p+1} f(x+\xi d) d^{p+1}\right| d \xi \\
& \leq T_{p}(x, d)+\frac{1}{p !}\left[\int_{0}^{1}(1-\xi)^{p} d \xi\right] \max _{\xi \in[0,1]}\left|\nabla^{p+1} f(x+\xi d) d^{p+1}\right| \\
& \leq T_{p}(x, d)+\frac{1}{(p+1) !}\|d\|^{p+1} \max _{\xi \in[0,1]}\left\|\nabla^{p+1} f(x+\xi d)\right\|_{T} \\
& \leq T_{p}(x, d)+\frac{M}{(p+1) !}\|d\|^{p+1} .
\end{aligned}
$$

Logo, a condição (2.16) vale para $\xi_{1}=M /(p+1)$ !. De maneira idêntica a (2.27), podemos expandir, usando Taylor, $\nabla f(x+d)$ e concluir que a condição (2.17) vale para $\xi_{2}=M / p$ !.

Nas próximas duas seções, apresentaremos dois algoritmos, bastante semelhantes, que usam a inspiração de modelos de ordem superior em seu desenvolvimento - o $\mathrm{AR}_{p}$ e o $\mathrm{AR}_{p}^{s}$.

\subsection{2 $\mathrm{O}$ algoritmo $\mathrm{AR}_{p}$}

O método que estudaremos nesta seção é apresentado em [10] e detalhado no Algoritmo 2.2.

Algoritmo 2.2: $\mathrm{AR}_{p}$

Passo 0. Inicialização. Dados $x_{0} \in \mathbb{R}^{n}$, um parâmetro regularizador inicial $\sigma_{0}>0$, uma precisão $\epsilon>0$ e as constantes positivas $\theta, \eta_{1}, \eta_{2}, \gamma_{1}, \gamma_{2}, \gamma_{3}$ e $\sigma_{\text {min }}$ tais que

$$
\sigma_{\min } \in\left(0, \sigma_{0}\right], 0<\eta_{1} \leq \eta_{2}<1 \text { e } 0<\gamma_{1}<1<\gamma_{2}<\gamma_{3},
$$

avalie $f\left(x^{0}\right)$ e inicialize $k \leftarrow 0$.

Passo 1. Critério de parada. Avalie $\nabla f\left(x^{k}\right)$. Se $\left\|\nabla f\left(x^{k}\right)\right\| \leq \epsilon$, defina $x^{\epsilon}=x^{k}$ e pare. Senão, avalie as derivadas de ordem 2 até $p$ de $f$ no ponto $x^{k}$. 
Passo 2. Cálculo do passo. Calcule o passo $d^{k}$ minimizando aproximadamente $m_{p}\left(x^{k}, d, \sigma_{k}\right)$ com respeito a $d$ de forma que satisfaça

$$
m_{p}\left(x^{k}, d^{k}, \sigma_{k}\right) \leq m_{p}\left(x^{k}, 0, \sigma_{k}\right)
$$

$\mathrm{e}$

$$
\left\|\nabla_{d} m_{p}\left(x^{k}, d^{k}, \sigma_{k}\right)\right\| \leq \theta\left\|d^{k}\right\|^{p} .
$$

Passo 3. Aceitação do ponto candidato. Avalie $f\left(x^{k}+d^{k}\right)$ e calcule

$$
\rho_{k}=\frac{f\left(x^{k}\right)-f\left(x^{k}+d^{k}\right)}{T_{p}\left(x^{k}, 0\right)-T_{p}\left(x^{k}, d^{k}\right)} .
$$

Se $\rho_{k} \geq \eta_{1}$, então $x^{k+1}=x^{k}+d^{k}$. Senão, defina $x^{k+1}=x^{k}$.

Passo 4. Atualização do parâmetro de regularização. Defina

$$
\sigma_{k+1} \in \begin{cases}{\left[\max \left\{\sigma_{\min }, \gamma_{1} \sigma_{k}\right\}, \sigma_{k}\right],} & \text { se } \rho_{k} \geq \eta_{2} \\ {\left[\sigma_{k}, \gamma_{2} \sigma_{k}\right],} & \text { se } \eta_{1} \leq \rho_{k}<\eta_{2} \\ {\left[\gamma_{2} \sigma_{k}, \gamma_{3} \sigma_{k}\right],} & \text { se } \rho_{k}<\eta_{1} .\end{cases}
$$

Faça $k \leftarrow k+1$ e vá ao Passo 1 , se $\rho_{k} \geq \eta_{1}$, ou ao Passo 2 caso contrário.

Notemos que no Passo 2 do Algoritmo 2.2 há uma minimização aproximada do modelo $m_{p}\left(x^{k}, d, \sigma_{k}\right)$, com respeito a $d$, de forma a cumprir as condições (2.28) e (2.29). Tais condições impõem apenas um decréscimo no modelo, o que significa que não requeremos aqui sua minimização global - um ponto aproximadamente estacionário de primeira ordem deve satisfazer tais condições. Além disso, a minimização aproximada do modelo não requer avaliação da função objetivo nem de suas derivadas, por conseguinte, o esforço computacional despendido no Passo 2 não afeta a complexidade de avaliação do Algoritmo 2.2.

Para continuarmos, é conveniente fixarmos algumas notações. As iterações para as quais $\rho_{k} \geq \eta_{1}$ (e, portanto, o ponto candidato é aceito) são chamadas de "bem sucedidas". Denotamos por $\mathcal{S}_{k} \stackrel{\text { def }}{=}\left\{0 \leq j \leq k \mid \rho_{j} \geq \eta_{1}\right\}$ o conjunto dos índices de todas as iterações bem sucedidas entre 0 e $k$. Não obstante, denotamos por $\mathcal{U}_{k}$ o complemento de $\mathcal{S}_{k}$ em $\{1,2, \ldots, k\}$, que corresponde ao conjunto dos índices de todas as iterações "mal sucedidas" entre 0 e $k$.

Para começarmos a nossa análise da complexidade de avaliação do Algoritmo 2.2, derivamos um resultado no decréscimo do modelo obtido sob a condição (2.28). É importante mencionar que as demonstrações dos lemas e teoremas desta subseção seguem argumentos padrão encontrados nos trabalhos de Nesterov [58,64], Nesterov e Polyak [59] e Cartis, Gould e Toint [20,27].

Lema 2.5. Para todo $k$,

$$
T_{p}\left(x^{k}, 0\right)-T_{p}\left(x^{k}, d^{k}\right) \geq \frac{\sigma_{k}}{p+1}\left\|d^{k}\right\|^{p+1} .
$$

Demonstração. De (2.28) e (2.18), temos que

$$
0 \leq m\left(x^{k}, 0, \sigma_{k}\right)-m\left(x^{k}, d^{k}, \sigma_{k}\right)=T_{p}\left(x^{k}, 0\right)-T_{p}\left(x^{k}, d^{k}\right)-\frac{\sigma_{k}}{p+1}\left\|d^{k}\right\|^{p+1},
$$

donde segue o resultado. 
Imediatamente obtemos do Lema 2.5 que o decréscimo em $T_{p}$ é não nulo e, portanto, que (2.30) está bem definido para todo $k$. Em seguida, deduziremos um limitante superior para o parâmetro regularizador $\sigma_{k}$.

Lema 2.6. Suponha que valha a Hipótese 2.3 para todo $x^{k}$ e todo $d^{k}$. Então, para todo $k \geq 0$,

$$
\sigma_{k} \leq \sigma_{\max } \stackrel{\text { def }}{=} \max \left\{\sigma_{0}, \gamma_{3}\left(\frac{\xi_{1}(p+1)}{1-\eta_{2}}\right)\right\}
$$

Demonstração. Suponhamos que

$$
\sigma_{k} \geq \frac{\xi_{1}(p+1)}{1-\eta_{2}}
$$

De (2.30), como $T_{p}\left(x^{k}, 0\right)=f\left(x^{k}\right)$, temos que

$$
\begin{aligned}
\rho_{k}-1 & =\frac{f\left(x^{k}\right)-f\left(x^{k}+d^{k}\right)-T_{p}\left(x^{k}, 0\right)+T_{p}\left(x^{k}, d^{k}\right)}{T_{p}\left(x^{k}, 0\right)-T_{p}\left(x^{k}, d^{k}\right)} \\
& =\frac{T_{p}\left(x^{k}, d^{k}\right)-f\left(x^{k}+d^{k}\right)}{T_{p}\left(x^{k}, 0\right)-T_{p}\left(x^{k}, d^{k}\right)} .
\end{aligned}
$$

Assim sendo, de (2.35), (2.24) e pelo Lema 2.5, temos que

$$
\left|\rho_{k}-1\right| \leq \frac{\left|f\left(x^{k}+d^{k}\right)-T_{p}\left(x^{k}, d^{k}\right)\right|}{\left|T_{p}\left(x^{k}, 0\right)-T_{p}\left(x^{k}, d^{k}\right)\right|} \leq \frac{\xi_{1}(p+1)}{\sigma_{k}} \leq 1-\eta_{2}
$$

e, por conseguinte, que $\rho_{k} \geq \eta_{2}$. Isso implica que a iteração $k$ é bem sucedida, ou seja, $\rho_{k} \geq \eta_{2}$ e $\sigma_{k+1} \leq \sigma_{k}$. Deste modo, o mecanismo de atualização do parâmetro regularizador no Passo 4 do Algoritmo 2.2, junto com a implicação de (2.34), garantem que (2.33) vale.

Agora, mostraremos que a direção calculada no Passo 2 do Algoritmo 2.2 não pode ser arbitrariamente pequena quando comparada ao gradiente da função objetivo no ponto candidato $x^{k}+d^{k}$.

Lema 2.7. Suponha que valha a Hipótese 2.3 para todo $x^{k}$ e todo $d^{k}$. Para todo $k \geq 0$,

$$
\left\|d^{k}\right\| \geq\left(\frac{\left\|\nabla f\left(x^{k}+d^{k}\right)\right\|}{\xi_{2}+\theta+\sigma_{k}}\right)^{\frac{1}{p}} .
$$

Demonstração. Como

$$
\left\|\nabla f\left(x^{k}+d^{k}\right)\right\|=\left\|\nabla f\left(x^{k}+d^{k}\right)-\nabla_{d} m_{p}\left(x^{k}, d^{k}, \sigma_{k}\right)+\nabla_{d} m_{p}\left(x^{k}, d^{k}, \sigma_{k}\right)\right\| .
$$

e o gradiente do modelo definido em (2.18) é dado por

$$
\nabla_{d} m_{p}(x, d, \sigma)=\nabla_{d} T_{p}(x, d)+\sigma\|d\|^{p} \frac{d}{\|d\|},
$$


então por (2.38) em (2.37), juntamente com a desigualdade triangular, (2.29) e (2.17), temos que

$$
\begin{aligned}
\left\|\nabla f\left(x^{k}+d^{k}\right)\right\| & \leq\left\|\nabla f\left(x^{k}+d^{k}\right)-\nabla_{d} T_{p}\left(x^{k}, d^{k}\right)\right\|+\left\|\nabla_{d} m_{p}\left(x^{k}, d^{k}, \sigma_{k}\right)\right\|+\sigma_{k}\left\|d^{k}\right\|^{p} \\
& \leq\left[\xi_{2}+\theta+\sigma_{k}\right]\left\|d^{k}\right\|^{p} .
\end{aligned}
$$

Segue o resultado.

A seguir, limitamos as iterações do Algoritmo 2.2 em função da quantidade de iterações bem sucedidas.

Lema 2.8. Para todo $k \geq 0$,

$$
k \leq\left|\mathcal{S}_{k}\right|\left(1+\frac{\left|\log \gamma_{1}\right|}{\log \gamma_{2}}\right)+\frac{1}{\log \gamma_{2}} \log \left(\frac{\sigma_{\max }}{\sigma_{0}}\right) .
$$

Demonstração. Pelas regras de atualização do parâmetro regularizador definidas em (2.31), temos que, para todo $k$,

$$
\gamma_{1} \sigma_{j} \leq \max \left\{\gamma_{1} \sigma_{j}, \sigma_{\min }\right\} \leq \sigma_{j+1}, j \in \mathcal{S}_{k} \text { e } \gamma_{2} \sigma_{j} \leq \sigma_{j+1}, j \in \mathcal{U}_{k}
$$

Portanto, indutivamente, podemos dizer que

$$
\sigma_{0} \gamma_{1}^{\left|\mathcal{S}_{k}\right|} \gamma_{2}^{\left|\mathcal{U}_{k}\right|} \leq \sigma_{k}
$$

Pelo Lema 2.6, de (2.40) segue que

$$
\left|\mathcal{S}_{k}\right| \log \gamma_{1}+\left|\mathcal{U}_{k}\right| \log \gamma_{2} \leq \log \left(\frac{\sigma_{\max }}{\sigma_{0}}\right)
$$

o que, como $\gamma_{2}>1$, implica que

$$
\left|\mathcal{U}_{k}\right| \leq-\left|\mathcal{S}_{k}\right| \frac{\log \gamma_{1}}{\log \gamma_{2}}+\frac{1}{\log \gamma_{2}} \log \left(\frac{\sigma_{\max }}{\sigma_{0}}\right)
$$

Não obstante, como $\gamma_{1}<1$ e $k=\left|\mathcal{S}_{k}\right|+\left|\mathcal{U}_{k}\right|$, de (2.41) segue o resultado.

Com os resultados obtidos até aqui, estamos prontos para estabelecer a complexidade de avaliação do Algoritmo 2.2. 
Teorema 2.9. Suponha que $f(x) \geq f_{\min }$, para todo $x \in \mathbb{R}^{n}$, e que valha a Hipótese 2.3 para todo $x^{k}$ e todo $d^{k}$. Então, para a precisão $\epsilon$ dada, o Algoritmo 2.2 consome, no máximo,

$$
\left\lfloor\left(\frac{f\left(x^{0}\right)-f_{\min }}{\kappa_{d}}\right) \epsilon^{-\frac{p+1}{p}}\right\rfloor
$$

iterações bem sucedidas e, no máximo

$$
\left\lfloor\left(\frac{f\left(x^{0}\right)-f_{\min }}{\kappa_{d}}\right) \epsilon^{-\frac{p+1}{p}}\right\rfloor\left(1+\frac{\left|\log \gamma_{1}\right|}{\log \gamma_{2}}\right)+\frac{1}{\log \gamma_{2}} \log \left(\frac{\sigma_{\max }}{\sigma_{0}}\right)
$$

iterações para produzir um iterando $x^{\epsilon}$ tal que $\left\|\nabla f\left(x^{\epsilon}\right)\right\| \leq \epsilon$, onde $\sigma_{\max }$ é dada no Lema 2.6 e

$$
\kappa_{d} \stackrel{\text { def }}{=} \frac{\eta_{1} \sigma_{\min }}{p+1}\left(\xi_{2}+\theta+\sigma_{\max }\right)^{-\frac{p+1}{p}} .
$$

Demonstração. A cada iteração bem sucedida, segue por (2.30) e pelo fato de que $\left\|\nabla f\left(x^{k}+d^{k}\right)\right\|>\epsilon$ enquanto o Algoritmo 2.2 não parar, que

$$
\begin{aligned}
f\left(x^{k}\right)-f\left(x^{k}+d^{k}\right) & \geq \eta_{1}\left[T_{p}\left(x^{k}, 0\right)-T_{p}\left(x^{k}, d^{k}\right)\right] \\
& \geq \frac{\eta_{1} \sigma_{\min }}{p+1}\left\|d^{k}\right\|^{p+1}, \text { por }(2.32) \text { e } \sigma_{k} \geq \sigma_{\min } \\
& \geq \frac{\eta_{1} \sigma_{\min }}{(p+1)\left(\xi_{2}+\theta+\sigma_{k}\right)^{\frac{p+1}{p}}}\left\|\nabla f\left(x^{k}+d^{k}\right)\right\|^{\frac{p+1}{p}}, \text { por }(2.36) \\
& >\frac{\eta_{1} \sigma_{\min }}{(p+1)\left(\xi_{2}+\theta+\sigma_{\max }\right)^{\frac{p+1}{p}}} \epsilon^{\frac{p+1}{p}}, \text { por }(2.33),
\end{aligned}
$$

donde segue que

$$
f\left(x^{k+1}\right)<f\left(x^{k}\right)-\kappa_{d} \epsilon^{\frac{p+1}{p}},
$$

com

$$
\kappa_{d}=\frac{\eta_{1} \sigma_{\min }}{p+1}\left(\xi_{2}+\theta+\sigma_{\max }\right)^{-\frac{p+1}{p}} .
$$

Como $f(x) \geq f_{\min }$, para todo $x \in \mathbb{R}^{n}$, temos que (2.44) pode ocorrer, no máximo,

$$
\left\lfloor\frac{f\left(x^{0}\right)-f_{\min }}{\kappa_{d} \epsilon^{\frac{p+1}{p}}}\right\rfloor
$$

vezes, o que implica (2.42). Não obstante, pelo Lema 2.8, considerando

$$
\left|\mathcal{S}_{k}\right| \leq\left\lfloor\left(\frac{f\left(x^{0}\right)-f_{\min }}{\kappa_{d}}\right) \epsilon^{-\frac{p+1}{p}}\right\rfloor
$$

em (2.39), segue (2.43).

Para completarmos as ideias desta seção, relembremos que, do Lema 2.5, concluímos que o decréscimo no modelo é não nulo, fazendo com que (2.31) esteja bem definido. Isto se deve ao fato de assumir que o parâmetro de regularização é não nulo durante toda a execução do algoritmo. $\mathrm{Na}$ próxima seção, apresentaremos uma segunda versão do Algoritmo 2.2, cuja principal diferença consiste na avaliação da aceitação do ponto candidato. A implicação maior desta modificação é justamente o fato de não requerer a não nulidade de $\sigma_{k}$, para todo $k$. 


\subsection{3 $\mathrm{O}$ algoritmo $\mathrm{AR}_{p}^{s}$}

Apresentaremos o algoritmo a seguir. Nesta descrição consta o algoritmo teórico, que itera indefinidamente, isto é, gera uma sequência infinita de iterandos. Na Seção 4.4.5 do Capítulo 4, apresentaremos os critérios de parada deste algoritmo.

\section{Algoritmo 2.3: $\mathrm{AR}_{p}^{s}$}

Entrada. Dados $x^{0} \in \mathbb{R}^{n}, \alpha>0, \eta_{1}, \eta_{2}>0, \sigma_{\min }>0, \theta>0, J \in \mathbb{N}$ e $0 \leq \gamma_{1} \leq 1<\gamma_{2}, \gamma_{3}$. Inicialize $\sigma_{-1}=0$ e $k \leftarrow 0$.

Passo 1. Defina $\sigma_{k, 0}=\gamma_{1} \sigma_{k-1}$, inicialize $j \leftarrow 0$ e escolha $J_{k} \in\{0,1,2, \ldots, J\}$.

Passo 2. Subproblema. Calcule, se possível, $d^{k, j}$ tal que

$$
m_{p}\left(x^{k}, d^{k, j}, \sigma_{k, j}\right) \leq m_{p}\left(x^{k}, 0, \sigma_{k, j}\right)
$$

e

$$
\left\|\nabla_{d} m_{p}\left(x^{k}, d^{k, j}, \sigma_{k, j}\right)\right\| \leq \theta\left\|d^{k, j}\right\|^{p} .
$$

Se não for possível calcular $d^{k, j}$ que satisfaça (2.45) e (2.46), defina $j=1, \sigma_{k, 1}=\sigma_{\min }$ e repita o Passo 2.

Passo 3. Controle de passo. Se

$$
j \geq J_{k} \text { ou }\left(\frac{T_{p}\left(x^{k}, 0\right)-T_{p}\left(x^{k}, d^{k, j}\right)}{\max \left\{1,\left|T_{p}\left(x^{k}, 0\right)\right|\right\}} \leq \eta_{1} \quad \text { e } \quad \frac{\left\|d^{k, j}\right\|_{\infty}}{\max \left\{1,\left\|x^{k}\right\|_{\infty}\right\}} \leq \eta_{2}\right)
$$

vá para o Passo 4. Caso contrário, defina $\sigma_{k, j+1}=\max \left\{\sigma_{\min }, \gamma_{2} \sigma_{k, j}\right\}, j \leftarrow j+1$ e vá para o Passo 2.

\section{Passo 4. Aceitação do ponto candidato. Se}

$$
f\left(x^{k}+d^{k, j}\right) \leq f\left(x^{k}\right)-\alpha\left\|d^{k, j}\right\|^{p+1},
$$

vá para o Passo 5. Senão, defina $\sigma_{k, j+1}=\max \left\{\sigma_{\min }, \gamma_{3} \sigma_{k, j}\right\}, j \leftarrow j+1$ e vá para o Passo 2 .

Passo 5. Defina $\sigma_{k}=\sigma_{k, j}, d^{k}=d^{k, j}$, and $x^{k+1}=x^{k}+d^{k}, k \leftarrow k+1$ e vá para o Passo 1.

No Algoritmo 2.3, inserimos uma estratégia de controle de passo. Neste contexto, vale comentar sobre as condições do Passo 3. Como $T_{p}\left(x^{k}, 0\right)=f\left(x^{k}\right)$, a condição

$$
\frac{T_{p}\left(x^{k}, 0\right)-T_{p}\left(x^{k}, d^{k, j}\right)}{\max \left\{1,\left|T_{p}\left(x^{k}, 0\right)\right|\right\}} \leq \eta_{1}
$$

descarta todo passo para o qual o decréscimo predito pelo modelo é muito maior que algo proporcional ao valor atual da função objetivo. Por outro lado, a condição

$$
\frac{\left\|d^{k, j}\right\|_{\infty}}{\max \left\{1,\left\|x^{k}\right\|_{\infty}\right\}} \leq \eta_{2},
$$


similar às condições clássicas de controle de passo usadas em métodos Newtonianos, trata de casos em que o passo gerado pela minimização do modelo é muito maior que a norma do iterando atual. Ambas as condições são indícios de que o modelo não deve ser uma boa aproximação para a função objetivo.

Essas condições foram incorporadas ao algoritmo com o objetivo de descartar passos para os quais o ponto candidato não seria aceito, por não cumprir a condição de decréscimo (2.48), sem sequer avaliar a função objetivo $f$. Vale ressaltar o limite máximo de iterações em que o controle de passo é executado, expresso na primeira parte da condição (2.47). Esta limitação é imposta a fim de preservar um limitante superior no parâmetro de regularização $\sigma_{k, j}$ (como demonstraremos adiante, no Corolário 2.11). O impacto prático do controle de passo será analisado posteriormente no Capítulo 4, em nossos experimentos numéricos.

A fim de seguirmos aos lemas e teoremas necessários para demonstrar a boa definição e complexidade de avaliação do Algoritmo 2.3, consideremos que $\|\cdot\|$ é uma norma arbitrária no $\mathbb{R}^{n}$. Vale destacar que a demonstração de complexidade do Algoritmo 2.3, como esperado, é similar à demonstração de complexidade do Algoritmo 2.2 .

Lema 2.10. Suponhamos que valha a Hipótese 2.3 para todo $x^{k}$ e todo $d^{k, j}$. Então, $d^{k}$ está bem definido para todo $k \geq 0$. Mais ainda, para todo $j \geq 0$, se

$$
\sigma_{k, j} \geq(p+1)\left(\xi_{1}+\alpha\right)
$$

então vale (2.48).

Demonstração. Por um lado, $m_{p}(x, d, \sigma)$ é, por construção, uma função suave de $d$ com conjuntos de nível limitados exceto, talvez, para $\sigma=0$. Portanto, o Passo 2 pode não estar bem definido apenas quando $\sigma=0$. Neste caso, o Passo 2 é repetido com $\sigma>0$. Portanto, $m_{p}\left(x^{k}, d, \sigma_{k, j}\right)$ admite, ao menos, um minimizador global, no qual seu gradiente é nulo e o valor mínimo é menor ou igual a $m_{p}\left(x^{k}, 0, \sigma_{k, j}\right)$. Portanto, existe ao menos um ponto que satisfaça (2.45) e (2.46).

Por outro lado, para todo $k \geq 0$ e $j \geq 0$ tais que valha (2.49), de (2.16), (2.18), e (2.45)

$$
\begin{aligned}
f\left(x^{k}+d^{k, j}\right) & \leq T_{p}\left(x^{k}, d^{k, j}\right)+\xi_{1}\left\|d^{k, j}\right\|^{p+1} \\
& =T_{p}\left(x^{k}, d^{k, j}\right)+\frac{\sigma_{k, j}}{p+1}\left\|d^{k, j}\right\|^{p+1}-\frac{\sigma_{k, j}}{p+1}\left\|d^{k, j}\right\|^{p+1}+\xi_{1}\left\|d^{k, j}\right\|^{p+1} \\
& =m_{p}\left(x^{k}, d^{k, j}, \sigma_{k, j}\right)-\left(\frac{\sigma_{k, j}}{p+1}-\xi_{1}\right)\left\|d^{k, j}\right\|^{p+1} \\
& \leq m_{p}\left(x^{k}, 0, \sigma_{k, j}\right)-\alpha\left\|d^{k, j}\right\|^{p+1} \\
& =f\left(x^{k}\right)-\alpha\left\|d^{k, j}\right\|^{p+1} .
\end{aligned}
$$

donde segue que vale (2.48). Portanto, pela estratégia de atualização do parâmetro de regularização $\sigma$, o Passo 4 itera uma quantidade finita de vezes.

Como $d^{k}$ está bem definido, para todo $k$, então o Lema 2.10 implica que o Algoritmo 2.3 está bem definido. Mais ainda, implica um resultado importante, que estabeleceremos a seguir. 
Corolário 2.11. Suponhamos que a Hipótese 2.3 valha para todo $x^{k}$ e todo $d^{k, j}$. Então, para todo $k$ e todo $j$,

$$
\sigma_{k, j} \leq \sigma_{\max } \stackrel{\text { def }}{=} \gamma_{2}^{J} \gamma_{3}(p+1)\left(\xi_{1}+\alpha\right)
$$

Além disto, para cada iteração $k$, os Passos 3 e 4 são executados, no máximo

$$
\kappa_{s} \stackrel{\text { def }}{=} \max \left\{J, \log _{\gamma}\left(\frac{\sigma_{\max }}{\sigma_{\min }}\right)+1\right\}
$$

vezes, onde $\gamma=\min \left\{\gamma_{2}, \gamma_{3}\right\}$

Demonstração. A primeira parte de (2.50) segue diretamente do Lema 2.10 e da estratégia de atualização do parâmetro de regularização $\sigma$ nos Passos 3 e 4.

Consideremos $\gamma=\min \left\{\gamma_{2}, \gamma_{3}\right\}$. Pela estratégia de atualização do parâmetro de regularização $\sigma$ nos Passos 3 e 4 do Algoritmo 2.3, temos que

$$
\sigma_{k, j} \geq \gamma^{j-1} \sigma_{\min }
$$

para todo $j \geq 1$. Logo, por (2.50),

$$
\gamma^{j-1} \sigma_{\min } \leq \sigma_{k, j} \leq \sigma_{\max }
$$

Por conseguinte,

$$
j \leq \log _{\gamma}\left(\frac{\sigma_{\max }}{\sigma_{\min }}\right)+1 .
$$

Isto, junto com (2.47), implica que (2.51) vale.

A seguir, mostramos que $d^{k}$, além de estar bem definida, possui norma limitada inferiormente por um valor que depende de constantes do Algoritmo 2.3 e da norma do gradiente da função objetivo no ponto candidato.

Lema 2.12. Suponhamos que a Hipótese 2.3 valha para todo $x^{k}$ e todo $d^{k, j}$. Então, para todo $k \in \mathbb{N}$,

$$
\left\|d^{k}\right\| \geq\left(\frac{\left\|\nabla f\left(x^{k}+d^{k}\right)\right\|}{\xi_{2}+\theta+\sigma_{\max }}\right)^{\frac{1}{p}} .
$$

Demonstração. Consideremos

$$
\left\|\nabla f\left(x^{k}+d^{k}\right)\right\|=\left\|\nabla f\left(x^{k}+d^{k}\right)-\nabla_{d} m_{p}\left(x^{k}, d^{k}, \sigma_{k}\right)+\nabla_{d} m_{p}\left(x^{k}, d^{k}, \sigma_{k}\right)\right\| .
$$

Como

$$
\nabla_{d} m_{p}(x, d, \sigma)=\nabla_{d} T_{p}(x, d)+\sigma\|d\|^{p} \frac{d}{\|d\|},
$$

substituindo (2.54) em (2.53), a desigualdade triangular, (2.17), (2.46) e o Corolário 2.11 implicam que

$$
\begin{aligned}
\left\|\nabla f\left(x^{k}+d^{k}\right)\right\| & \leq\left\|\nabla f\left(x^{k}+d^{k}\right)-\nabla_{d} T_{p}\left(x^{k}, d^{k}\right)\right\|+\left\|\nabla_{d} m_{p}\left(x^{k}, d^{k}, \sigma_{k}\right)\right\|+\sigma_{k}\left\|d^{k}\right\|^{p} \\
& \leq\left(\xi_{2}+\theta+\sigma_{\max }\right)\left\|d^{k}\right\|^{p}
\end{aligned}
$$

donde segue que (2.52) vale. 
Temos agora todos os ingredientes para estabelecer o resultado de complexidade do Algoritmo 2.3.

Teorema 2.13. Suponhamos que a Hipótese 2.3 valha para todo $x^{k}$ e todo $d^{k, j}$ e que $f(x) \geq f_{\min }$, para todo $x \in \mathbb{R}^{n}$. Dado $\epsilon>0$, o Algoritmo 2.3 consome, no máximo,

$$
k_{\text {up }} \stackrel{\text { def }}{=}\left\lfloor\left[\frac{\left(\xi_{2}+\theta+\sigma_{\max }\right)^{\frac{p+1}{p}}}{\alpha}\right]\left(\frac{f\left(x^{0}\right)-f_{\min }}{\epsilon^{\frac{p+1}{p}}}\right)\right\rfloor
$$

iterações e, no máximo,

$$
\left\lfloor\kappa_{s} k_{\mathrm{up}}\right\rfloor
$$

avaliações de função para convergir a um iterando $x^{\epsilon}$ tal que $\left\|\nabla f\left(x^{\epsilon}\right)\right\| \leq \epsilon$, onde $\kappa_{s}$ é definido em (2.51).

Demonstração. A cada iteração do Algoritmo 2.3, temos que vale (2.48). Isto, o Lema $2.12 \mathrm{e}$ o fato que $\left\|\nabla f\left(x^{k}+d^{k}\right)\right\|>\epsilon$ antes do Algoritmo 2.3 convergir a um ponto aproximadamente estacionário, implicam que

$$
\begin{aligned}
f\left(x^{k}+d^{k}\right) & \leq f\left(x^{k}\right)-\alpha\left\|d^{k}\right\|^{p+1} \\
& \leq f\left(x^{k}\right)-\alpha\left(\frac{\epsilon}{\xi_{2}+\theta+\sigma_{\max }}\right)^{\frac{p+1}{p}} .
\end{aligned}
$$

Como $f(x) \geq f_{\min }$ para todo $x \in \mathbb{R}^{n}$, (2.55) segue de (2.57), dividindo-se $f(x)-f_{\min }$ pelo decréscimo fixo obtido a cada iteração expresso em (2.57). Pelo Corolário 2.11, podemos limitar o valor de $j$ para cada $k$, multiplicando (2.51) por (2.55) e obtendo, portanto, (2.56).

Vale dizer que $\sigma_{\min }$ é um parâmetro do algoritmo, dado pelo usuário, e que não é uma cota inferior para a sequência de parâmetros de regularização $\left\{\sigma_{k}\right\}$ gerada pelo Algoritmo 2.3. O papel de $\sigma_{\min }$ é garantir que o parâmetro de regularização $\sigma$ saia de zero caso seja necessário aumentá-lo a partir do zero nos Passos 3 e 4 . Notemos que, caso $\sigma_{k}=0$ para todo $k$, então o número máximo de repetições $j$ por iteração $k$ dos Passos 3 e 4 do Algoritmo 1, isto é, $\kappa_{s}$ definida em (2.51), será 1. Em outras palavras, neste caso os Passos 3 e 4 serão executados apenas uma vez a cada iteração externa $k$.

\subsubsection{Considerações sobre as duas abordagens}

As duas abordagens descritas nas duas últimas seções são bastante similares, todavia os Algoritmos 2.2 e 2.3 diferem em dois pontos principais.

Em primeiro lugar, o critério de aceitação do ponto candidato no Passo 3 do $\mathrm{AR}_{p}$ e no Passo 4 do $\mathrm{AR}_{p}^{s}$. O critério (2.30) no $\mathrm{AR}_{p}$ é livre de escala, isto é, o polinômio de Taylor possui a mesma escala da função objetivo e, portanto, $\rho_{k}$ será um valor adimensional. Isso traz a vantagem de que a aceitação do ponto candidato independe de características do problema. Por outro lado, o critério (2.48) não é livre de escala, portanto, a aceitação do ponto candidato está condicionada à características do problema, além da escolha do valor da constante $\alpha$.

Em segundo lugar, a regra de atualização do parâmetro regularizador no Passo 4 do $\mathrm{AR}_{p}$ e no Passo 5 do $\mathrm{AR}_{p}^{s}$. $\mathrm{O}$ algoritmo $\mathrm{AR}_{p}^{s}$ admite parâmetro de regularização nulo, isso sem afetar a sua complexidade de avaliação, enquanto que em (2.31) o valor do parâmetro de regularização não 
assume valores menores que $\sigma_{\min }>0$. Há certas vantagens de se permitir parâmetro de regularização nulo.

Para ilustrar bem esta vantagem, consideremos o caso em que $p=2$. Neste caso, a cada iteração de ambos os algoritmos minimizamos aproximadamente, em $d$, o modelo

$$
m_{2}\left(x^{k}, d, \sigma_{k}\right)=f\left(x^{k}\right)+\nabla f\left(x^{k}\right)^{T} d+\frac{1}{2} d^{T} \nabla^{2} f\left(x^{k}\right) d+\frac{1}{3} \sigma_{k}\|d\|^{3} .
$$

Se $\sigma_{k}=0$ e $\nabla^{2} f\left(x^{k}\right)$ for definida positiva, então $m_{2}$ é uma quadrática convexa e a solução de minimizar $m_{2}\left(x^{k}, d, 0\right)$ é o próprio passo de Newton. Por conseguinte, para $p=2$, o $\mathrm{AR}_{p}^{s}$ tem chances de dar o passo de Newton quando próximo à solução; o mesmo não acontece com o $\mathrm{AR}_{p}$ por não admitir valores nulos para $\sigma_{k}$ nem tampouco que $\sigma_{k}$ decresça muito rapidamente (por limitar, em (2.31), o decrescimento do parâmetro regularizador pela fração $\left.\gamma_{1}\right)$. 


\section{Capítulo 3}

\section{Complexidade em otimização com restrições}

Neste capítulo, estamos interessados em estudar a complexidade de métodos para o problema geral de programação não linear

$$
\begin{aligned}
\text { Minimizar } & f(x) \\
\text { s. a } & h(x)=0 \\
& g(x) \leq 0,
\end{aligned}
$$

onde $f: \mathbb{R}^{n} \rightarrow \mathbb{R}, h: \mathbb{R}^{n} \rightarrow \mathbb{R}^{m}$ e $g: \mathbb{R}^{n} \rightarrow \mathbb{R}^{q}$ são funções $p$ vezes continuamente diferenciáveis.

Em [23, 27, 28], Cartis, Gould e Toint propuseram um método de primeira ordem de duas fases baseado em regiões de confiança que, em $O\left(\epsilon^{-2}\right)$ avaliações de função (objetivo e de restrições, e seus respectivos gradientes) convergia a um ponto aproximadamente estacionário de primeira ordem, isto é, um ponto que satisfaz (1.1). O uso de informações de segunda ordem foi avaliado em [26], onde mostrou-se que um método de duas fases, similar ao anteriormente proposto pelos mesmos autores, consumia $O\left(\epsilon^{-3 / 2}\right.$ ) avaliações de função (objetivo, gradiente e Hessiana) para computar um ponto que satisfaz condições similares de otimalidade.

Neste capítulo, apresentaremos um método, publicado em [9], que, inspirado na proposta de Cartis, Gould e Toint $[27,28]$, explora modelos de ordem superior e converge a pontos que satisfazem condições de otimalidade de primeira ordem não escaladas para o problema (3.1) consumindo entre $O\left(\epsilon^{1-2(p+1) / p}\right)$ e $O\left(\epsilon^{1-3(p+1) / p}\right)$ avaliações funcionais.

Notação. Antes de definir o algoritmo, para uma função vetorial $v(\cdot)$ assumiremos a notação

$$
v(x)_{+} \stackrel{\text { def }}{=} \max \{v(x), 0\}
$$

onde o máximo é feito componente a componente. Além disso, $\nabla v(x)$ denotará o gradiente de $v(\cdot)$ com relação a $x$, e a notação $[x]_{j}$ será usada para indicar a $j$-ésima componente do vetor $x$ quando a notação $x_{j}$ for ambígua.

Definimos

$$
V(x) \stackrel{\text { def }}{=}\|h(x)\|^{2}+\left\|g(x)_{+}\right\|^{2}
$$

a violação das restrições em um ponto $x$ e, para um dado $\nu>0$, o conjunto de nível como

$$
\mathcal{L}(\nu) \stackrel{\text { def }}{=}\left\{x \in \mathbb{R}^{n} \mid V(x) \leq \nu\right\} .
$$

Além disso, para um dado $t \in \mathbb{R}$, consideraremos

$$
\Phi(x, t) \stackrel{\text { def }}{=} V(x)+[f(x)-t]_{+}^{2},
$$


sendo $t$ nosso alvo (do inglês, target). Consideraremos $\nabla \Phi(x, t)$ o gradiente de $\Phi(\cdot, \cdot)$ com relação a $x$.

\section{$3.1 \quad \mathrm{O}$ algoritmo}

O algoritmo FTARget (do inglês, Feasibility and Target algorithm) é um algoritmo de duas fases que, em sua primeira fase, obtém viabilidade aproximada e, sem perdê-la na fase seguinte (veja Lema 3.1 adiante), busca otimalidade usando um método de target following, isto é, para um dado alvo $t$, sem perder viabilidade, buscamos decrescer a função objetivo até atingir o valor $t$. Na segunda fase, o método computa iterandos $\left\{x^{k}\right\}$ por meio de iterações externas. Para obter um novo ponto $x^{k+1}$, resolvemos um problema de minimização irrestrita usando um algoritmo para minimização irrestrita qualquer que compute, por meio de iterações internas, iterandos $x^{k, j}$.

\section{Algoritmo FTARGET}

Entrada. Dados $\epsilon_{\mathrm{P}} \in(0,1]$ a precisão para otimalidade (precisão primal) e $\epsilon_{\mathrm{D}} \in(0,1]$ e precisão para viabilidade (precisão dual), $\rho \in(0,1)$ e um ponto inicial $x^{-1} \in \mathbb{R}^{n}$. Considere ainda $\psi: \mathbb{R}_{+} \rightarrow \mathbb{R}_{+}$uma função contínua e não decrescente tal que $\psi(0)=0$. Consideremos ainda que $f, g$ e $h$ são $p$ vezes continuamente diferenciáveis.

Fase 1. Computação de um ponto inicial aproximadamente viável

Passo F1. Minimize $V(x)$ usando um algoritmo de minimização irrestrita que gere uma sequência monotonicamente decrescente, começando de $x^{-1,0}=x^{-1}$, para computar um iterando $x^{-1, j}, j \in\{0,1,2, \ldots\}$, tal que $V\left(x^{-1, j}\right) \leq V\left(x^{-1,0}\right)$ e pelo menos uma das duas seguintes condições seja satisfeita:

$$
\begin{gathered}
V\left(x^{-1, j}\right) \leq 0,99 \epsilon_{\mathrm{P}}^{2} \\
V\left(x^{-1, j}\right)>0,99 \epsilon_{\mathrm{P}}^{2} \text { e }\left\|\nabla V\left(x^{-1, j}\right)\right\| \leq \psi\left(\epsilon_{\mathrm{D}}\right) .
\end{gathered}
$$

Defina $x^{0}=x^{-1, j}$.

Passo F2. Se $V\left(x^{0}\right)>0,99 \epsilon_{\mathrm{P}}^{2}$ e $\left\|\nabla V\left(x^{0}\right)\right\| \leq \psi\left(\epsilon_{\mathrm{D}}\right)$, pare o Algoritmo FTARGET e retorne $x^{\epsilon}=x^{0}$.

Fase 2. Otimização target following

Passo T0. Inicialize $k \leftarrow 0$.

Passo T1. Compute $t_{k}=f\left(x^{k}\right)-\sqrt{\epsilon_{\mathrm{P}}^{2}-V\left(x^{k}\right)}$.

Passo T2. Minimize $\Phi\left(x, t_{k}\right)$ usando um algoritmo para minimização irrestrita que gere uma sequência monotonicamente decrescente, começando de $x^{k, 0}=x^{k}$, para computar um iterando $x^{k, j}, j \in\{0,1,2, \ldots\}$, tal que $\Phi\left(x^{k, j}, t_{k}\right) \leq \Phi\left(x^{k, 0}, t_{k}\right)$ e pelo menos uma das três seguintes condições seja satisfeita:

$$
\begin{gathered}
f\left(x^{k, j}\right) \leq t_{k}+\rho\left(f\left(x^{k}\right)-t_{k}\right) \text { e } V\left(x^{k, j}\right) \leq 0,99 \epsilon_{\mathrm{P}}^{2}, \\
f\left(x^{k, j}\right)>t_{k} \text { e }\left\|\nabla \Phi\left(x^{k . j}, t_{k}\right)\right\| \leq 2 \epsilon_{\mathrm{D}}\left[f\left(x^{k, j}\right)-t_{k}\right]_{+}, \\
V\left(x^{k, j}\right)>0,99 \epsilon_{\mathrm{P}}^{2} \text { e }\left\|\nabla V\left(x^{k, j}\right)\right\| \leq \psi\left(\epsilon_{\mathrm{D}}\right) .
\end{gathered}
$$

Defina $x^{k+1}=x^{k, j}$. 
Passo T3. Se $V\left(x^{k+1}\right)>0,99 \epsilon_{\mathrm{P}}^{2}$ e $\left\|\nabla V\left(x^{k+1}\right)\right\| \leq \psi\left(\epsilon_{\mathrm{D}}\right)$, pare o Algoritmo FTARGet e retorne $x^{\epsilon}=x^{k+1}$.

Passo T4. Se $f\left(x^{k+1}\right)>t_{k}$ e $\left\|\nabla \Phi\left(x^{k+1}, t_{k}\right)\right\| \leq 2 \epsilon_{\mathrm{D}}\left[f\left(x^{k+1}\right)-t_{k}\right]_{+}$, pare o Algoritmo FTARGET e retorne $x^{\epsilon}=x^{k+1}$.

Passo T5. Faça $k \leftarrow k+1$ e vá para o Passo T1.

Para justificar a descrição do método dada no parágrafo anterior, enunciaremos o seguinte lema.

Lema 3.1. Para todo $k \in\{0,1,2, \ldots\}$ temos que

$$
V\left(x^{k}\right) \leq \epsilon_{\mathrm{P}}^{2}
$$

para todo iterando $x^{k}$ computado na Fase 2 do Algoritmo FTARget.

Demonstração. Mostraremos por indução em $k$. Para $k=0$, se o Algoritmo FTARGET avançou para a Fase 2, então a condição (3.4) foi satisfeita no Passo F1. Por conseguinte, vale que $V\left(x^{0}\right) \leq \epsilon_{\mathrm{P}}^{2}$. Suponhamos então que vale $V\left(x^{k}\right) \leq \epsilon_{\mathrm{P}}^{2}$ para $k \geq 0$. Pela definição de $t_{k}$ no Passo T1 do Algoritmo FTarget temos que

$$
\begin{aligned}
\Phi\left(x^{k, 0}, t_{k}\right) & =\Phi\left(x^{k}, t_{k}\right) \\
& =V\left(x^{k}\right)+\left[f\left(x^{k}\right)-t_{k}\right]_{+}^{2} \\
& =V\left(x^{k}\right)+\left[f\left(x^{k}\right)-f\left(x^{k}\right)+\sqrt{\epsilon_{\mathrm{P}}^{2}-V\left(x^{k}\right)}\right]_{+}^{2} \\
& =\epsilon_{\mathrm{P}}^{2} .
\end{aligned}
$$

Portanto, como no Passo T2 o algoritmo de minimização irrestrita empregado retornará $x^{k, j}=$ $x^{k+1}$ tal que $\Phi\left(x^{k, j}, t_{k}\right) \leq \Phi\left(x^{k, 0}, t_{k}\right)$, vem que

$$
V\left(x^{k+1}\right)+\left[f\left(x^{k+1}\right)-t_{k}\right]_{+}^{2}=\Phi\left(x^{k, j}, t_{k}\right) \leq \Phi\left(x^{k, 0}, t_{k}\right)=\epsilon_{\mathrm{P}}^{2},
$$

ou seja,

$$
V\left(x^{k+1}\right) \leq \epsilon_{\mathrm{P}}^{2}
$$

Segue o resultado.

O Algoritmo FTARget é um arcabouço, isto é, depende das escolhas específicas da ordem de derivação $p \geq 1$ e do algoritmo de minimização irrestrita para desempenhar o papel de minimização no Passo F1 e Passo T2. Para cada possível escolha, o algoritmo pode possuir comportamento completamente distinto.

Analisaremos o Algoritmo FTARget da seguinte forma. Primeiro, discutiremos sobre o significado de cada critério de parada do algoritmo sob a ótica do que esperar do ponto retornado em algumas escolhas algorítmicas específicas. Em seguida, demonstraremos a boa definição e a complexidade de avaliação do Algoritmo FTARGET à luz de algumas suposições sobre o algoritmo para minimização irrestrita escolhido para a resolução dos subproblemas. 


\subsection{Sobre os critérios de parada}

A fim de expor a discussão sobre os critérios de parada, primeiro discorreremos de um modo geral sobre todos os critérios de parada, apresentando uma abordagem mais minuciosa logo em seguida.

Parar no Passo T4 significa que, no limite, um ponto aproximadamente KKT (AKKT) foi encontrado. Parar no Passo F2 tem dois significados possíveis: (i) um ponto inviável estacionário do problema de inviabilidade foi identificado no limite e (ii) uma situação análoga à parada do Passo T3 aconteceu. A interpretação da parada no Passo T3 depende de algumas condições fracas nas tolerâncias $\epsilon_{\mathrm{P}}$ e $\epsilon_{\mathrm{D}}$ no limite e, ainda mais importante, na escolha da função $\psi$. Para três escolhas chave distintas para a função $\psi$, mostraremos que parar no Passo T3 significa que (i) um ponto $\epsilon_{\mathrm{P}}$-viável $z$ foi encontrado tal que os gradientes das restrições ativas em $z$ não são uniformemente linearmente independentes (com coeficientes não negativos para as restrições de desigualdade); (ii) um ponto viável que não satisfaz a condição de qualificação de Mangasarian-Fromovitz (MFCQ) existe no limite ou (iii) um ponto viável para o qual a medida de violação das restrições não satisfaz a desigualdade de Łojasiewicz existe no limite. Nós poderemos concluir então que, para as três opções consideradas para a função $\psi$, FTARGET sempre converge a um ponto aproximadamente KKT não escalado sob certas hipóteses de "não degenerescência". Todas essas afirmações serão objetos da nossa exposição nas próximas seções.

\subsubsection{Parada no Passo T4}

Quando o Algoritmo FTARget para na iteração $k$ satisfazendo o critério de parada do Passo T4, retorna-se um ponto $x^{\epsilon}=x^{k+1}$ tal que $f\left(x^{k+1}\right)>t_{k}$ e

$$
\left\|\nabla \Phi\left(x^{k+1}, t_{k}\right)\right\| \leq 2 \epsilon_{\mathrm{D}}\left[f\left(x^{k+1}\right)-t_{k}\right]_{+} .
$$

Pela definição de $\Phi(\cdot, \cdot)$ em (3.3), temos que

$$
\begin{aligned}
\nabla \Phi(x, t) & =\nabla V(x)+2[f(x)-t]_{+} \nabla f(x) \\
& =2\left[\sum_{i=1}^{m} h_{i}(x) \nabla h_{i}(x)+\sum_{j=1}^{q}\left[g_{j}(x)\right]_{+} \nabla g_{j}(x)+[f(x)-t]_{+} \nabla f(x)\right]
\end{aligned}
$$

Por conseguinte, como $f\left(x^{k+1}\right)-t_{k}>0$, de (3.9) temos que

$$
\left\|\nabla f\left(x^{k+1}\right)+\sum_{i=1}^{m} \lambda_{i} \nabla h_{i}\left(x^{k+1}\right)+\sum_{j=1}^{q} \mu_{j} \nabla g_{j}\left(x^{k+1}\right)\right\| \leq \epsilon_{\mathrm{D}},
$$

onde

$$
\lambda_{i}=\frac{h_{i}\left(x^{k+1}\right)}{f\left(x^{k+1}\right)-t_{k}} \text { e } \mu_{j}=\frac{\left[g_{j}\left(x^{k+1}\right)\right]_{+}}{f\left(x^{k+1}\right)-t_{k}} .
$$

Portanto, temos que se $g_{j}\left(x^{k+1}\right) \leq 0$, então $\left[g_{j}\left(x^{k+1}\right)\right]_{+}=0$ e, por isso, $\mu_{j}=0$, o que implica que

$$
\min \left\{\mu_{j},-g_{j}\left(x^{k+1}\right)\right\}=\mu_{j}=0 .
$$

Por outro lado, se $g_{j}\left(x^{k+1}\right)>0$, então

$$
\min \left\{\mu_{j},-g_{j}\left(x^{k+1}\right)\right\}=-g_{j}\left(x^{k+1}\right) \leq \epsilon_{\mathrm{P}}
$$


As relações (3.11) e (3.12) implicam que a complementaridade (medida com a função min) é satisfeita com precisão $\epsilon_{\mathrm{P}}$. Logo, o Lema 3.1 e as equações (3.10), (3.11) e (3.12), implicam que as condições AKKT valem em $x^{k+1}$ com precisão $\max \left\{\epsilon_{\mathrm{P}}, \epsilon_{\mathrm{D}}\right\}$.

É conveniente inserirmos aqui a seguinte definição, extraída de [14, p. 17].

Definição 3.1. (AKKT) Dizemos que $x^{*} \in \mathbb{R}^{n}$ satisfaz as condições aproximadas de KarushKuhn-Tucker (AKKT) com relação ao problema de programação não linear definido em (3.1) se existem sequências $\left\{x^{k}\right\} \subseteq \mathbb{R}^{n},\left\{\lambda^{k}\right\} \subseteq \mathbb{R}^{m}$ e $\left\{\mu^{k}\right\} \subseteq \mathbb{R}_{+}^{q}$ tais que

$$
\begin{gathered}
\lim _{k \rightarrow \infty} x^{k}=x^{*} \\
\lim _{k \rightarrow \infty}\left\|\nabla f\left(x^{k}\right)+\sum_{i=1}^{m} \lambda_{i}^{k} \nabla h_{i}\left(x^{k}\right)+\sum_{j=1}^{q} \mu_{j}^{k} \nabla g_{j}\left(x^{k}\right)\right\|=0
\end{gathered}
$$

$e$

$$
\lim _{k \rightarrow \infty} \min \left\{\mu_{j}^{k},-g_{j}\left(x^{k}\right)\right\}=0 \text { para todo } j=1, \ldots, q
$$

Agora, analisaremos o que acontece em termos assintóticos, isto é, se assumirmos que FTARGET é executado indefinidamente com $\epsilon_{\mathrm{P}}=\epsilon_{\mathrm{P}, \ell} \rightarrow 0$ e $\epsilon_{\mathrm{D}}=\epsilon_{\mathrm{D}, \ell} \rightarrow 0$, e que ele para em todas as execuções $\left(\ell_{1}, \ell_{2}, \ldots\right)$ retornando um ponto $x^{\epsilon}\left(\ell_{i}\right), i=1,2, \ldots$ satisfazendo os critérios de parada do Passo T4. Sob tais circunstâncias, temos, para qualquer ponto de acumulação $z$ da sequência $\left\{x^{\epsilon}\left(\ell_{i}\right)\right\}$, que

$$
\lim _{i_{s} \rightarrow \infty} x^{\epsilon}\left(\ell_{i_{s}}\right)=z
$$

para alguma subsequência $\left\{i_{s}\right\} \subseteq\{i\}$ e, pelo Lema 3.1, por (3.10), (3.11) e (3.12), que

$$
\begin{gathered}
\lim _{i_{s} \rightarrow \infty} V\left(x^{\epsilon}\left(\ell_{i_{s}}\right)\right)=0 \\
\lim _{i_{s} \rightarrow \infty}\left\|\nabla f\left(x^{\epsilon}\left(\ell_{i_{s}}\right)\right)+\sum_{i=1}^{m} \lambda_{i} \nabla h_{i}\left(x^{\epsilon}\left(\ell_{i_{s}}\right)\right)+\sum_{j=1}^{q} \mu_{j} \nabla g_{j}\left(x^{\epsilon}\left(\ell_{i_{s}}\right)\right)\right\|=0
\end{gathered}
$$

e

$$
\lim _{i_{s} \rightarrow \infty} \min \left\{\mu_{j},-g_{j}\left(x^{\epsilon}\left(\ell_{i_{s}}\right)\right)\right\}=0 .
$$

Pela Definição 3.1 e pelas relações (3.13)-(3.16), temos que $z$ é um ponto AKKT.

\subsubsection{Parada no Passo F2 ou no Passo T3}

Estudar as consequências de parada no Passo F2 ou no Passo T3 requer uma análise acerca da escolha e interpretação da função $\psi$. Esta função é usada para interromper a execução do Algoritmo FTArget no Passo F2 e no Passo T3 quando a medida de inviabilidade é "grande" (isto é, quando $V(x) \geq 0,99 \epsilon_{\mathrm{P}}^{2}$ ) e o gradiente da medida de inviabilidade é pequeno (ou seja, quando $\|\nabla V(x)\| \leq$ $\left.\psi\left(\epsilon_{\mathrm{D}}\right)\right)$. À primeira vista, este comportamento indica que $x$ é um "ponto inviável aproximadamente estacionário de $V(x)$ ". Não obstante, uma análise cuidadosa de alguns casos específicos mostram algumas sutilezas não aparentes à primeira vista. 
Quando o Algoritmo FTARget para no Passo F2, é retornado um ponto $x^{\epsilon}$ tal que

$$
V\left(x^{\epsilon}\right)>0,99 \epsilon_{\mathrm{P}}^{2} \text { e }\left\|\nabla V\left(x^{\epsilon}\right)\right\| \leq \psi\left(\epsilon_{\mathrm{D}}\right) .
$$

Neste ponto, é adequado pensarmos nestas condições em termos assintóticos. Por isso, assumamos que FTARget é executado indefinidamente com $\epsilon_{\mathrm{P}}=\epsilon_{\mathrm{P}, \ell} \rightarrow 0$ e $\epsilon_{\mathrm{D}}=\epsilon_{\mathrm{D}, \ell} \rightarrow 0$. Além disso, assumamos que ele para em todas as execuções $\ell_{1}, \ell_{2}, \ldots$ no Passo F2, retornando $x^{\epsilon}\left(\ell_{i}\right), i=1,2, \ldots$, tal que $\lim _{i \rightarrow \infty} x^{\epsilon}\left(\ell_{i}\right)=z$. De (3.17) temos que

$$
\lim _{i \rightarrow \infty}\left\|\nabla V\left(x^{\epsilon}\left(\ell_{i}\right)\right)\right\|=0
$$

Se $V\left(x^{\epsilon}\left(\ell_{i}\right)\right)$ não convergir nem se aproximar, indefinidamente, de zero, por (3.18) parar no Passo F2 significa que $z$ é um ponto inviável para o problema (3.1) e é estacionário da medida de inviabilidade $V$. Se, por outro lado, $\lim _{i \rightarrow \infty} V\left(x^{\epsilon}\left(\ell_{i}\right)\right)=0$, então a interpretação deste fato depende da escolha da função $\psi$ e possui a mesma análise do caso em que o Algoritmo FTARGET para no Passo T3, caso que analisaremos a seguir.

Quando o Algoritmo FTArget para no Passo T3, ele retorna um ponto $x^{\epsilon}$ tal que

$$
0,99 \epsilon_{\mathrm{P}}^{2}<V\left(x^{\epsilon}\right) \leq \epsilon_{\mathrm{P}}^{2} \quad \text { e }\left\|\nabla V\left(x^{\epsilon}\right)\right\| \leq \psi\left(\epsilon_{\mathrm{D}}\right) .
$$

A interpretação deste critério de parada está inerentemente ligada à escolha da função $\psi$. Consideraremos, então, funções $\psi$ da forma

$$
\psi\left(\epsilon_{\mathrm{D}}\right)=\sigma_{1} \epsilon_{\mathrm{D}}^{\sigma_{2}},
$$

com $\sigma_{1}>0$ e três possibilidades para $\sigma_{2}$ : (a) $\sigma_{2}=1$, (b) $\sigma_{2} \in(1,2)$ e (c) $\sigma_{2}=2$. Notemos que $\psi$ assim definida satisfaz as condições necessárias do Algoritmo FTARGET: é uma função contínua, não decrescente e $\psi(0)=0$.

No caso (a), (3.19) implica que

$$
\frac{\left\|\nabla V\left(x^{\epsilon}\right)\right\|}{\sqrt{V\left(x^{\epsilon}\right)}}<\frac{\sigma_{1}}{\sqrt{0,99}} \frac{\epsilon_{\mathrm{D}}}{\epsilon_{\mathrm{P}}}
$$

Pela definição $(3.2)$ de $V(\cdot)$, temos que

$$
\frac{\left\|\nabla V\left(x^{\epsilon}\right)\right\|}{\sqrt{V\left(x^{\epsilon}\right)}}=2\left\|\sum_{i=1}^{m} \lambda_{i} \nabla h_{i}\left(x^{\epsilon}\right)+\sum_{j=1}^{q} \mu_{j} \nabla g_{j}\left(x^{\epsilon}\right)\right\|,
$$

onde

$$
\lambda_{i}=\frac{h_{i}\left(x^{\epsilon}\right)}{\sqrt{\left\|h\left(x^{\epsilon}\right)\right\|^{2}+\left\|g\left(x^{\epsilon}\right)_{+}\right\|^{2}}}, \text { para } i=1, \ldots, m
$$

e

$$
\mu_{j}=\frac{\left[g_{j}\left(x^{\epsilon}\right)\right]_{+}}{\sqrt{\left\|h\left(x^{\epsilon}\right)\right\|^{2}+\left\|g\left(x^{\epsilon}\right)_{+}\right\|^{2}}}, \text { para } j=1, \ldots, q
$$

Notemos que, por (3.23) e (3.24),

$$
\sum_{i=1}^{m} \lambda_{i}^{2}+\sum_{j=1}^{q} \mu_{j}^{2}=1 \text { e } \mu_{j}=0 \text { sempre que } g_{j}\left(x^{\epsilon}\right)<0 .
$$

Cabe aqui a seguinte definição. 
Definição 3.2. Dado $\xi>0$, dizemos que $x$ possui gradientes $\xi$-uniformemente positivamente linearmente independentes com respeito ao problema (3.1) se para todo $(\lambda, \mu) \in \mathbb{R}^{m} \times \mathbb{R}_{+}^{q}$ tal que $\sum_{i=1}^{m} \lambda_{i}^{2}+\sum_{j=1}^{q} \mu_{j}^{2}=1$ e $\mu_{j}=0$ sempre que $g_{j}(x)<0$, temos que

$$
\left\|\sum_{i=1}^{m} \lambda_{i} \nabla h_{i}(x)+\sum_{j=1}^{q} \mu_{j} \nabla g_{j}(x)\right\| \geq \xi .
$$

Assim sendo, por (3.21), podemos dizer que o ponto $x^{\epsilon}$ retornado pelo Algoritmo FTARGET não possui gradiente das restrições ativas $\xi$-uniformemente positivamente linearmente independentes, com

$$
\xi=\frac{\sigma_{1}}{2 \sqrt{0,99}} \frac{\epsilon_{\mathrm{D}}}{\epsilon_{\mathrm{P}}}
$$

Se o quociente $\sigma_{1} \epsilon_{\mathrm{D}} / \epsilon_{\mathrm{P}}$ for suficientemente pequeno, isso significa que uma condição de qualificação aproximada de Mangasarian-Fromovitz com tolerância $\xi$ não é satisfeita em $x^{\epsilon}$.

As análises dos casos (b) e (c) derivam resultados interessantes se feitas assintoticamente. Então, novamente, assumamos que o Algoritmo FTARGET é executado indefinidamente com $\epsilon_{\mathrm{P}}=\epsilon_{\mathrm{P}, \ell} \rightarrow 0$ e $\epsilon_{\mathrm{D}}=\epsilon_{\mathrm{D}, \ell} \rightarrow 0$. Assumamos ainda que ele para em todas as execuções $\ell_{1}, \ell_{2}, \ldots$ no Passo T3 retornando $x^{\epsilon}\left(\ell_{i}\right), i=1,2, \ldots$, tal que $\lim _{i \rightarrow \infty} x^{\epsilon}\left(\ell_{i}\right)=z$. Assumiremos, por fim, para o resto desta seção, que

$$
\omega \stackrel{\text { def }}{=} \limsup _{i \rightarrow \infty} \frac{\epsilon_{\mathrm{D}, \ell_{i}}}{\epsilon_{\mathrm{P}, \ell_{i}}}<\infty
$$

Nos casos (b) e (c), por (3.19), temos que

$$
\frac{\left\|\nabla V\left(x^{\epsilon}\left(\ell_{i}\right)\right)\right\|}{\sqrt{V\left(x^{\epsilon}\left(\ell_{i}\right)\right.}}<\frac{\sigma_{1}}{\sqrt{0,99}} \frac{\epsilon_{\mathrm{D}, \ell_{i}}^{\sigma_{2}}}{\epsilon_{\mathrm{P}, \ell_{i}}}, \text { para } i=1,2, \ldots
$$

Tomando o limite em (3.27) e usando (3.26) e o fato de que $\sigma_{2}>1$, temos, por (3.22)-(3.25) que os gradientes das restrições ativas não são positivamente linearmente independentes e, por conseguinte, MFCQ não vale no ponto viável $z$ [61].

Consequentemente, se o conjunto viável for compacto e todos os pontos viáveis satisfizerem MFCQ então, para $i$ suficientemente grande, parar no Passo T3 é impossível de acontecer quando valer (3.26). Portanto, a Fase 2 do Algoritmo FTARget para apenas no Passo T4, convergindo a um ponto AKKT.

O caso (c) ainda possui algumas particularidades. Para discorrer sobre elas, é importante introduzir a seguinte definição, a desigualdade de Łojasiewicz [51].

Definição 3.3. (Desigualdade de Eojasiewicz) Uma função continuamente diferenciável $v: \mathbb{R}^{n} \rightarrow \mathbb{R}$ satisfaz a desigualdade de Lojasiewicz em $\bar{x}$ se existe $\delta>0, \tau \in(0,1)$ e $\kappa>0$ tais que, para todo $x \in \mathcal{B}(\bar{x}, \delta)$,

$$
|v(x)-v(\bar{x})|^{\tau} \leq \kappa\|\nabla v(x)\| .
$$


As propriedades das funções que satisfazem (3.28) tem sido alvo de estudos nos últimos anos em vários artigos relacionados a métodos para minimização, teoria de complexidade, análise assintótica de equações diferenciais parciais e a satisfação de condições AKKT em métodos de Lagrangianos aumentados [2,18]. Sabe-se que funções suaves satisfazem a desigualdade de Łojasiewicz sob hipóteses fracas. Por exemplo, funções analíticas satisfazem a desigualdade de Łojasiewicz (veja [51]).

No caso (c), por (3.19) temos que

$$
\frac{\left\|\nabla V\left(x^{\epsilon}\left(\ell_{i}\right)\right)\right\|}{V\left(x^{\epsilon}\left(\ell_{i}\right)\right)}<\frac{\sigma_{1}}{0,99}\left(\frac{\epsilon_{\mathrm{D}, \ell_{i}}}{\epsilon_{\mathrm{P}, \ell_{i}}}\right)^{2}, \text { para } i=1,2, \ldots
$$

Portanto, para um $\tau \in(0,1)$ qualquer, temos que

$$
V\left(x^{\epsilon}\left(\ell_{i}\right)\right)^{\tau}=V\left(x^{\epsilon}\left(\ell_{i}\right)\right)^{\tau-1} V\left(x^{\epsilon}\left(\ell_{i}\right)\right)>V\left(x^{\epsilon}\left(\ell_{i}\right)\right)^{\tau-1}\left[\frac{\sigma_{1}}{0,99}\left(\frac{\epsilon_{\mathrm{D}, \ell_{i}}}{\epsilon_{\mathrm{P}, \ell_{i}}}\right)^{2}\right]^{-1}\left\|\nabla V\left(x^{\epsilon}\left(\ell_{i}\right)\right)\right\| .
$$

Como, por (3.19), $V\left(x^{\epsilon}\left(\ell_{i}\right)\right) \leq \epsilon_{\mathrm{P}, \ell_{i}}^{2}$, então $V\left(x^{\epsilon}\left(\ell_{i}\right)\right) \rightarrow 0$ quando $i$ tende ao infinito. Por conseguinte, $V\left(x^{\epsilon}\left(\ell_{i}\right)\right)^{\tau-1}$ tende ao infinito, já que $\tau \in(0,1)$ e, portanto, usando (3.26), para todo $\delta>0$ e $\kappa>0$ existe $i$ suficientemente grande tal que

$$
x^{\epsilon}\left(\ell_{i}\right) \in \mathcal{B}(z, \delta) \text { e } V\left(x^{\epsilon}\left(\ell_{i}\right)\right)^{\tau}>\kappa\left\|\nabla V\left(x^{\epsilon}\left(\ell_{i}\right)\right)\right\| .
$$

Isso significa que a função $V(\cdot)$ não satisfaz a desigualdade de Łojasiewicz em nenhum ponto limite $z$. Portanto, se assumirmos que a função $V(\cdot)$ satisfaz a desigualdade de Łojasiewicz em todo ponto limite viável e que $j$ é suficientemente grande, então, se (3.26) valer, a Fase 2 do Algoritmo FTARget só para no Passo T4, retornando, em consequência, um ponto AKKT.

\subsection{Boa definição, convergência e complexidade}

Nesta seção, demonstraremos que o Algoritmo FTARGET está bem definido e termina sua execução em um número finito de iterações desde que o algoritmo de minimização irrestrita escolhido para minimizar $V(x)$ no Passo F1 e para minimizar $\Phi\left(x, t_{k}\right)$ no Passo T2 possua propriedades de convergência global. Ainda mais, se o algoritmo de minimização irrestrita possuir propriedades adequadas de complexidade de avaliação, podemos estabelecer a complexidade de avaliação do Algoritmo FTARGET em si.

Para começar, estabeleceremos algumas hipóteses essenciais para o desenvolvimento desta seção.

Hipótese 3.1. Existe uma constante $f_{\min }$ tal que $f(x) \geq f_{\min }$ para todo $x \in \mathcal{L}\left(\epsilon_{\mathrm{p}}^{2}\right)$.

Hipótese 3.2. Existe uma constante $\kappa_{\nabla}$ tal que $\|\nabla f(x)\| \leq \kappa_{\nabla}$ para todo $x \in \mathcal{L}\left(\epsilon_{\mathrm{P}}^{2}\right)$.

Para facilitar nossa notação adiante, suporemos que

$$
\kappa_{\nabla} \geq \max \left\{1, \frac{5 \sigma_{1}}{2 \rho}\right\}
$$


A Hipótese 3.1 é suficiente para provar um primeiro resultado bastante importante para o estudo da complexidade do Algoritmo FTARget. No Lema 3.2 mostraremos que, independentemente do algoritmo para minimização irrestrita empregado no Passo F1 e Passo T2 do Algoritmo FTARget, o número de iterações externas não excede um limitante superior dependente apenas de $\epsilon_{\mathrm{P}}$ e de $f_{\min }$.

Lema 3.2. Suponha que valha a Hipótese 3.1. Então o Algoritmo FTARGET para na Fase 1 ou efetua, no máximo,

$$
\left\lfloor\frac{f\left(x^{0}\right)-f_{\min }}{0.1(1-\rho) \epsilon_{\mathrm{P}}}\right\rfloor+1
$$

iterações externas na Fase 2.

Demonstração. Suponhamos que o Algoritmo FTARget não pare na Fase 1. Pela definição do algoritmo, uma iteração externa é iniciada apenas quando $V\left(x^{k}\right) \leq 0,99 \epsilon_{\mathrm{p}}^{2}$ e é seguida por outra iteração externa somente quando vale (3.6), ou seja, quando

$$
f\left(x^{k+1}\right) \leq t_{k}+\rho\left(f\left(x^{k}\right)-t_{k}\right) \text { e } V\left(x^{k+1}\right) \leq 0,99 \epsilon_{\mathrm{p}}^{2} .
$$

Pela definição de $t_{k}$ no Passo T1 do Algoritmo FTArget, temos que

$$
\begin{aligned}
f\left(x^{k+1}\right) & \leq t_{k}+\rho \sqrt{\epsilon_{\mathrm{P}}^{2}-V\left(x^{k}\right)} \\
& =f\left(x^{k}\right)-\sqrt{\epsilon_{\mathrm{P}}^{2}-V\left(x^{k}\right)}+\rho \sqrt{\epsilon_{\mathrm{P}}^{2}-V\left(x^{k}\right)} \\
& =f\left(x^{k}\right)-(1-\rho) \sqrt{\epsilon_{\mathrm{P}}^{2}-V\left(x^{k}\right) .}
\end{aligned}
$$

Portanto, como $V\left(x^{k}\right) \leq 0,99 \epsilon_{\mathrm{P}}^{2}$,

$$
f\left(x^{k+1}\right) \leq f\left(x^{k}\right)-(1-\rho) \sqrt{\epsilon_{\mathrm{P}}^{2}-0,99 \epsilon_{\mathrm{P}}^{2}}=f\left(x^{k}\right)-0.1(1-\rho) \epsilon_{\mathrm{P}} .
$$

Em outras palavras, quando uma iteração externa é completada satisfazendo (3.6), obtemos um decréscimo de pelo menos $0.1(1-\rho) \epsilon_{\mathrm{P}}$ na função objetivo. Portanto, como, pela Hipótese 3.1, $f(x) \geq f_{\text {min }}$ para todo $x \in \mathcal{L}\left(\epsilon_{\mathrm{P}}^{2}\right)$, a quantidade total de iterações completadas satisfazendo (3.6) deve ser menor ou igual que

$$
\left\lfloor\frac{f\left(x^{0}\right)-f_{\min }}{0.1(1-\rho) \epsilon_{\mathrm{P}}}\right\rfloor
$$

O resultado segue adicionando-se uma iteração final em que (3.6) pode não valer.

Observe que, de acordo com o Lema 3.2, o número de iterações efetuadas pelo Algoritmo FTARGET poderia ser apenas um. Este caso ocorreria se $\epsilon_{\mathrm{P}}$ fosse suficientemente grande e, consequentemente, $t_{0}$ fosse muito pequeno.

Agora, precisamos demonstrar que uma vez que uma iteração externa é iniciada, ela será completada em uma quantidade finita de iterações internas. O Lema 3.3 estabelece que se $V\left(x^{k}\right) \leq 0,99 \epsilon_{\mathrm{P}}^{2}$ e o algoritmo de minimização irrestrita não para no ponto $x^{k, j}$, então a norma $\left\|\nabla \Phi\left(x^{k, j}, t_{k}\right)\right\|$ é afastada de zero por uma quantidade que depende apenas das constantes $\epsilon_{\mathrm{P}}, \epsilon_{\mathrm{D}}$ e um limitante na norma do gradiente de $f$ em $\mathcal{L}\left(\epsilon_{\mathrm{P}}^{2}\right)$. 
Lema 3.3. Suponha que a Hipótese 3.2 valha, que $V\left(x^{k}\right) \leq 0,99 \epsilon_{\mathrm{P}}^{2}$ e que o algoritmo para minimização irrestrita usado para minimizar $\Phi\left(x, t_{k}\right)$ não para no iterando interno $x^{k, j}$. Então,

$$
\left\|\nabla \Phi\left(x^{k, j}, t_{k}\right)\right\| \geq \min \left\{0.2 \rho \epsilon_{\mathrm{P}} \epsilon_{\mathrm{D}}, \frac{\psi\left(\epsilon_{\mathrm{D}}\right)}{2}, \frac{\psi\left(\epsilon_{\mathrm{D}}\right) \epsilon_{\mathrm{D}}}{2 \kappa_{\nabla}}\right\} .
$$

Demonstração. Como o algoritmo de minimização irrestrita não para no iterando $x^{k, j}$, temos que nenhuma das relações (3.6)-(3.8) vale. Consideraremos, então, dois casos:

$$
f\left(x^{k, j}\right)>t_{k}+\rho\left(f\left(x^{k}\right)-t_{k}\right)
$$

e

$$
f\left(x^{k, j}\right) \leq t_{k}+\rho\left(f\left(x^{k}\right)-t_{k}\right)
$$

Consideremos o caso (3.32) em primeiro lugar. Dele, segue que $f\left(x^{k, j}\right)>t_{k}$. Como (3.7) não vale, temos que

$$
\left\|\nabla \Phi\left(x^{k, j}, t_{k}\right)\right\|>2 \epsilon_{\mathrm{D}}\left(f\left(x^{k, j}\right)-t_{k}\right) .
$$

Por (3.32) e pela definição de $t_{k}$ no Passo T1 do Algoritmo FTARGET, dado que $V\left(x^{k}\right) \leq 0,99 \epsilon_{\mathrm{P}}^{2}$, vem que

$$
f\left(x^{k, j}\right)-t_{k}>\rho\left(f\left(x^{k}\right)-t_{k}\right)>\rho \sqrt{\epsilon_{\mathrm{P}}^{2}-V\left(x^{k}\right)} \geq 0.1 \rho \epsilon_{\mathrm{P}} .
$$

Por conseguinte, por (3.34),

$$
\left\|\nabla \Phi\left(x^{k, j}, t_{k}\right)\right\|>0.2 \rho \epsilon_{\mathrm{P}} \epsilon_{\mathrm{D}}
$$

Por sua vez, consideremos o caso em que vale (3.33). Portanto, $V\left(x^{k}\right)>0,99 \epsilon_{\mathrm{P}}^{2}$, caso contrário, (3.6) teria sido satisfeito. Logo, como (3.8) também não vale, temos que

$$
\begin{aligned}
\psi\left(\epsilon_{\mathrm{D}}\right) & <\left\|\nabla V\left(x^{k, j}\right)\right\| \\
& =\left\|\nabla V\left(x^{k, j}\right)+2\left[f\left(x^{k, j}\right)-t_{k}\right]_{+} \nabla f\left(x^{k, j}\right)-2\left[f\left(x^{k, j}\right)-t_{k}\right]_{+} \nabla f\left(x^{k, j}\right)\right\|_{1} \\
& \leq\left\|\nabla V\left(x^{k, j}\right)+2\left[f\left(x^{k, j}\right)-t_{k}\right]_{+} \nabla f\left(x^{k, j}\right)\right\|+2\left\|\nabla f\left(x^{k, j}\right)\right\|\left[f\left(x^{k, j}\right)-t_{k}\right]_{+} \\
& \leq\left\|\nabla \Phi\left(x^{k, j}, t_{k}\right)\right\|+2 \kappa_{\nabla}\left[f\left(x^{k, j}\right)-t_{k}\right]_{+},
\end{aligned}
$$

onde usamos a monotonicidade do algoritmo de minimização irrestrita (implicando que $x^{k, j} \in$ $\left.\mathcal{L}\left(\epsilon_{\mathrm{P}}^{2}\right)\right)$ e a Hipótese 3.2 para obter a última desigualdade. Disso segue que

$$
\left\|\nabla \Phi\left(x^{k, j}, t_{k}\right)\right\|>\psi\left(\epsilon_{\mathrm{D}}\right)-2 \kappa_{\nabla}\left[f\left(x^{k, j}\right)-t_{k}\right]_{+} .
$$

Diante disto, consideraremos dois casos,

$$
2 \kappa_{\nabla}\left[f\left(x^{k, j}\right)-t_{k}\right]_{+} \leq \frac{\psi\left(\epsilon_{\mathrm{D}}\right)}{2}
$$

e seu complementar

$$
2 \kappa_{\nabla}\left[f\left(x^{k, j}\right)-t_{k}\right]_{+}>\frac{\psi\left(\epsilon_{\mathrm{D}}\right)}{2} .
$$

Por (3.37), de (3.36) segue que

$$
\left\|\nabla \Phi\left(x^{k, j}, t_{k}\right)\right\|>\frac{\psi\left(\epsilon_{\mathrm{D}}\right)}{2} .
$$


Por outro lado, por (3.38), temos que

$$
2\left[f\left(x^{k, j}\right)-t_{k}\right]_{+}>\frac{\psi\left(\epsilon_{\mathrm{D}}\right)}{2 \kappa_{\nabla}} .
$$

Como (3.7) não vale,

$$
\left\|\nabla \Phi\left(x^{k . j}, t_{k}\right)\right\|>2 \epsilon_{\mathrm{D}}\left[f\left(x^{k, j}\right)-t_{k}\right]_{+}>\frac{\psi\left(\epsilon_{\mathrm{D}}\right) \epsilon_{\mathrm{D}}}{2 \kappa_{\nabla}} .
$$

As desigualdades (3.35), (3.39) e (3.40) implicam o resultado.

Todos os ingredientes formulados até aqui nos permitem começar a análise de convergência do Algoritmo FTARget. Para tanto, formularemos uma hipótese acerca da convergência global do algoritmo de minimização irrestrita empregado nos subproblemas de minimização oriundos do Passo F1 e Passo T2 do Algoritmo FTARGet. Esta hipótese expressa que o algoritmo monotônico para minimização irrestrita possui convergência global de primeira ordem no sentido de que a sequência de iterandos $\left\{x^{j}\right\}$ por ele gerada, quando aplicada à minimização de uma função limitada inferiormente, possui uma subsequência para a qual o gradiente de tal função converge a zero. Formularemos esta hipótese de uma maneira adequada à aplicação da teoria de convergência do Algoritmo FTARGET.

Hipótese 3.3. Para um $\varepsilon>0$ arbitrário, temos que se o algoritmo de minimização irrestrita é aplicado à minimização de $V(x)$ e de $\Phi(x, t)$ com relação a $x$, começando ambas de um ponto inicial qualquer $x^{0}$, então, em uma quantidade finita de iterações, este algoritmo encontra um iterando $x$ tal que $V(x) \leq V\left(x^{0}\right)$ e $\|\nabla V(x)\| \leq \varepsilon$, ou $\Phi(x, t)<\Phi\left(x^{0}, t\right)$ e $\|\nabla \Phi(x, t)\| \leq \varepsilon$, respectivamente.

Com a Hipótese 3.3 em mãos, juntamente com a Hipótese 3.2, estabeleceremos no Lema 3.4 que, dado o iterando $x^{k}$ do Algoritmo FTARGET, o iterando $x^{k+1}$ está bem definido e é computado em uma quantidade finita de iterações.

Lema 3.4. Suponha que as Hipóteses 3.2 e 3.3 valham. Então, para todo $k \in\{0,1, \ldots\}$, o algoritmo para minimização irrestrita aplicado à minimização de $\Phi\left(x, t_{k}\right)$ encontra um ponto $x^{k, j}$ que satisfaz ao menos um dos critérios (3.6)-(3.8) em uma quantidade finita de iterações.

Demonstração. Pela Hipótese 3.3, o algoritmo para minimização irrestrita encontra um ponto $x^{k, j_{0}}$, para algum $j_{0} \geq 0$, que satisfaz $\left\|\nabla \Phi\left(x^{k, j_{0}}\right)\right\| \leq \varepsilon$, para algum $\varepsilon>0$ arbitrário. Consideremos, então,

$$
\varepsilon=\min \left\{0.2 \rho \epsilon_{\mathrm{P}} \epsilon_{\mathrm{D}}, \frac{\psi\left(\epsilon_{\mathrm{D}}\right)}{2}, \frac{\psi\left(\epsilon_{\mathrm{D}}\right) \epsilon_{\mathrm{D}}}{2 \kappa_{\nabla}}\right\}>0 .
$$

Pelo Lema 3.3 e pela definição de $\varepsilon, x^{k, j_{0}}$ satisfaz (3.6), (3.7) ou (3.8), e a iteração $k$ do Algoritmo FTARGET termina em algum $x^{k, j}, \operatorname{com} j \leq j_{0}$.

Tudo isto nos permite agora demonstrar a terminação finita do Algoritmo FTARGET. 
Teorema 3.5. Suponha que as Hipóteses 3.1-3.3 valham. Então, o Algoritmo FTARGET para no Passo F2 da Fase 1 ou, no máximo, depois de

$$
\left\lfloor\frac{f\left(x^{0}\right)-f_{\min }}{0.1(1-\rho) \epsilon_{\mathrm{P}}}\right\rfloor+1
$$

iterações da Fase 2 com $x^{k+1}$ satisfazendo o critério do Passo T3 ou Passo T4.

Demonstração. Pela Hipótese 3.3, o problema de minimização do Passo F1 da Fase 1 é resolvido pelo algoritmo de minimização irrestrita em uma quantidade finita de iterações. Pelo Lema 3.2, o Algoritmo FTARget efetua, no máximo, (3.41) iterações externas. Pelo Lema 3.4, cada iteração externa está bem definida e termina em tempo finito. Por conseguinte, o Algoritmo FTARget para no Passo F2 da Fase 1 ou para na última iteração da Fase 2, caso em que, pela própria definição do método, $x^{k+1}$ deve satisfazer o critério do Passo T3 ou do Passo T4.

Neste ponto, iniciamos o estudo da complexidade de avaliação do Algoritmo FTARGET. Para tanto, introduziremos uma hipótese adicional sobre o algoritmo de minimização irrestrita empregado no Passo F1 e Passo T2 do Algoritmo FTARget. Enquanto a Hipótese 3.3 diz que, para qualquer $\varepsilon>0$, o algoritmo de minimização irrestrita converge a um ponto $x$ que cumpre $\|\nabla V(x)\| \leq \varepsilon$ ou $\left\|\nabla \Phi\left(x, t_{k}\right)\right\| \leq \varepsilon$ em uma quantidade finita de iterações, a Hipótese 3.4 diz respeito à quantidade de avaliações funcionais consumidas para convergir a um ponto com norma de gradiente suficientemente pequena.

Hipótese 3.4. Existe um $\alpha \geq 0$ e uma constante $\kappa_{V}>0$ (dependente de propriedades das funções $h$ e $g$ e de parâmetros do algoritmo para minimização irrestrita) tais que, dado $\varepsilon>0$, se o algoritmo para minimização irrestrita é aplicado para minimização de $V(x)$ iniciando de um ponto inicial arbitrário $x^{-1}$, o algoritmo encontra um iterando $x$ tal que $V(x) \leq V\left(x^{-1}\right)$ e $\|\nabla V(x)\| \leq \varepsilon$ efetuando, no máximo,

$$
\kappa_{V}\left[\frac{V\left(x^{-1}\right)}{\varepsilon^{\alpha}}\right]
$$

avaliações de $h$ e $g$ e de suas derivadas. Mais ainda, se, para qualquer $t \in \mathbb{R}$ o algoritmo para minimização irrestrita é aplicado à minimização de $\Phi(x, t)$ (com respeito a $x$ ) partindo de um ponto inicial arbitrário $x^{0}$, então existe uma constante $\kappa_{\Phi}$ (dependente de propriedades das funções $f$, h e $g$ e dos parâmetros do algoritmo para minimização irrestrita) tal que o algoritmo encontra um iterando $x$ tal que $\Phi(x, t) \leq \Phi\left(x^{0}, t\right)$ e $\|\nabla \Phi(x, t)\| \leq \varepsilon$ efetuando, no máximo,

$$
\kappa_{\Phi}\left[\frac{\Phi\left(x^{0}, t\right)}{\varepsilon^{\alpha}}\right]
$$

avaliações de $f, g$ e $h$ e de suas derivadas.

Note que as derivadas usadas no algoritmo para minimização irrestrita podem ser aproximadas, se necessário, dado que tal aproximação seja precisa o suficiente para garantir uma taxa de convergência global. (Veja, por exemplo, [25] ou [63] para exemplos de algoritmos derivative-free adequados.) 
Vale aqui comentar um pouco mais a respeito do que significa a Hipótese 3.4 do ponto de vista de alguns exemplos de métodos conhecidos. As constantes $\kappa_{V}$ e $\kappa_{\Phi}$ mencionadas na hipótese dependem de parâmetros do algoritmo para minimização irrestrita e em grandezas associadas com a função objetivo $(V$ e $\Phi)$ do problema de minimização irrestrita a ser resolvido. Por exemplo, se o algoritmo para minimização irrestrita empregado é um método de primeira ordem com busca linear típico e $L_{\Phi}$ é uma constante de Lipschitz para $\nabla \Phi(x, t)$ (para todo $t$ ), temos que $\alpha=2$ e $\kappa_{\Phi}=L_{\Phi} \kappa_{M}$, onde $\kappa_{M}$ depende de parâmetros do algoritmo, como tolerâncias para decréscimo suficiente e constantes relacionadas às condições do ângulo e da norma (veja, por exemplo, a Seção 2.1 do Capítulo 2 ou [32]). Note que assumir que exista $L_{\Phi}$ constante de Lipschitz de $\nabla \Phi(x, t)$ independente do valor de $t$ é uma consideração plausível se assumirmos a continuidade Lipschitz de $\nabla f, \nabla h$ e $\nabla g$. Conclusões similares poderiam ser tiradas se considerássemos o método de regularização cúbica adaptativo (ARC) $[21,22]$ ao invés de um método de descida de primeira ordem.

Todavia, pelo interesse em entender quais implicações ocorreriam por diferentes escolhas algorítmicas para o método de minimização irrestrita, chegamos a conclusões levemente diferentes para um caso em que desejarmos explorar derivadas de ordem superior com a expectativa de obter um método com melhor resultado de complexidade de avaliação no pior caso. Neste ponto, remetemos ao trabalho de Birgin et. al. [10], ou à Seção 2.2 do Capítulo 2 da presente tese, onde problemas de minimização irrestrita usando modelos de ordem superior são considerados. Como conclusões do método proposto neste trabalho, suponha que estivéssemos interessados em minimizar a função

$$
u(x)=v(x)+w(x),
$$

onde $v(x)$ é ao menos uma vez continuamente diferenciável e $w(x)$ é $p$ vezes continuamente diferenciável com sua $p$-ésima derivada Lipschitz contínua. Se as derivadas até ordem $p$ estiverem disponíveis, surge uma variante do ARC que, iniciando de um ponto $\bar{x}$ e empregando modelos de ordem superior certamente produzirá um ponto aproximadamente crítico de primeira ordem (isto é, $\|\nabla u(x)\| \leq \varepsilon)$ em uma quantidade de avaliações de $w(x)$ e de suas derivadas de, no máximo,

$$
\kappa_{A}\left[\frac{u(\bar{x})-u_{\min }}{\varepsilon^{(p+1) / p}}\right]
$$

onde $u_{\text {min }}$ é um limitante inferior global para $u(x)$ e $\kappa_{A}$ depende apenas da constante de Lipschitz da $p$-ésima derivada de $w$, de $p$ e dos parâmetros algorítmicos. Para poder aplicar esta técnica, é necessário reformular o problema (3.1) a uma forma equivalente dada por

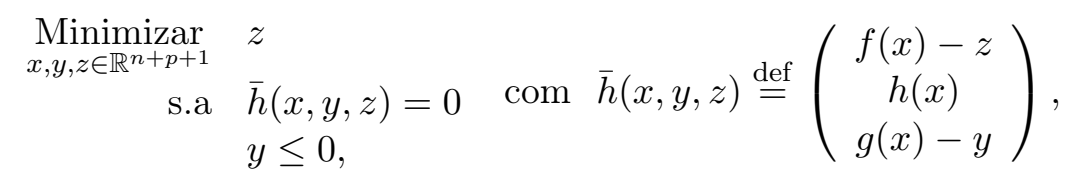

e construir a função $\Phi(x, y, z, t)$ associada dada por

$$
\Phi(x, y, z, t)=\underbrace{\|\bar{h}(x, y, z)\|^{2}}_{w(x, y, z)}+\underbrace{\left\|y_{+}\right\|^{2}+[z-t]_{+}^{2}}_{v(y, z, t)} .
$$

Note que $v(y, z, t)$ é uma vez continuamente diferenciável e que as propriedades de diferenciabilidade de $f, g$ e $h$ são transferidas à $\bar{h}(x, y, z)$ : a $p$-ésima derivada de $\Phi(x, y, z, t)$ com relação às suas três primeiras variáveis é Lipschitz contínua se todas as derivadas de $f, g$ e $h$ forem limitadas até ordem $p-1$ e se a $p$-ésima derivada for Lipschitz contínua. Note também que a avaliação de $v(y, z, t)$ não envolve avaliação da função objetivo nem tampouco das funções das restrições, e por conseguinte a quantidade de avaliações de $v(y, z, t)$ não interfere na complexidade de avaliação do Algoritmo FTARGET. Nestas condições, podemos concluir que o algoritmo para minimização com modelos de 
ordem superior apresentado na Seção 2.2 da presente tese (ou em [10]) satisfaz a Hipótese 3.4 com $\kappa_{V}$ e $\kappa_{\Phi}$ igual a $\kappa_{A}$ e $\alpha=(p+1) / p$.

Neste momento, reformularemos o Lema 3.4 de forma a quantificar as avaliações funcionais consumidas pelo algoritmo para minimização irrestrita empregado no Algoritmo FTARGET.

Lema 3.6. Suponha que as Hipóteses 3.1, 3.2 e 3.4 valham. Então, para todo $k \geq 0$, o algoritmo para minimização irrestrita aplicado à minimização de $\Phi\left(x, t_{k}\right)$ encontra um ponto $x^{k, j}$ que satisfaz ao menos uma das condições (3.6)-(3.8) consumindo não mais que

$$
\left\lfloor\kappa_{\Phi}\left(\frac{2 \kappa_{\nabla}}{\sigma_{1}}\right)^{\alpha} \epsilon_{\mathrm{P}}^{2} \epsilon_{\mathrm{D}}^{-\alpha} \min \left\{\epsilon_{\mathrm{D}}^{\sigma_{2}}, \epsilon_{\mathrm{P}}\right\}^{-\alpha}\right\rfloor
$$

avaliações de $f, g$ e $h$, e suas derivadas.

Demonstração. Sabendo que $\Phi\left(x^{k, 0}, t_{k}\right)=\Phi\left(x^{k}, t_{k}\right)=\epsilon_{\mathrm{P}}^{2}$ e usando o Lema 3.3 e a Hipótese 3.4, deduzimos que o algoritmo para minimização irrestrita não consome mais que

$$
\kappa_{\Phi}\left[\frac{\epsilon_{\mathrm{P}}^{2}}{\min \left\{0.2 \rho \epsilon_{\mathrm{P}} \epsilon_{\mathrm{D}}, \frac{\psi\left(\epsilon_{\mathrm{D}}\right)}{2}, \frac{\psi\left(\epsilon_{\mathrm{D}}\right) \epsilon_{\mathrm{D}}}{2 \kappa_{\nabla}}\right\}^{\alpha}}\right]
$$

avaliações funcionais para convergir a um minimizador aproximado do problema. Como $\epsilon_{\mathrm{D}} \leq 1$ e, por (3.29), $\kappa_{\nabla} \geq 1$, certamente

$$
\frac{\psi\left(\epsilon_{\mathrm{D}}\right) \epsilon_{\mathrm{D}}}{2 \kappa_{\nabla}} \leq \frac{\psi\left(\epsilon_{\mathrm{D}}\right)}{2}
$$

o que, junto com (3.43), implica que

$$
\min \left\{0.2 \rho \epsilon_{\mathrm{P}} \epsilon_{\mathrm{D}}, \frac{\psi\left(\epsilon_{\mathrm{D}}\right)}{2}, \frac{\psi\left(\epsilon_{\mathrm{D}}\right) \epsilon_{\mathrm{D}}}{2 \kappa_{\nabla}}\right\}=\min \left\{0.2 \rho \epsilon_{\mathrm{P}} \epsilon_{\mathrm{D}}, \frac{\psi\left(\epsilon_{\mathrm{D}}\right) \epsilon_{\mathrm{D}}}{2 \kappa_{\nabla}}\right\} .
$$

Não obstante, ainda por (3.29), temos que

$$
\kappa_{\nabla} \geq \frac{5 \sigma_{1}}{2 \rho}
$$

donde segue que

$$
\rho \geq \frac{5 \sigma_{1}}{2 \kappa_{\nabla}} .
$$

Deste limitante em $\rho$ em (3.44), juntamente com (3.20), segue que

$$
\begin{aligned}
\min \left\{0.2 \rho \epsilon_{\mathrm{P}} \epsilon_{\mathrm{D}}, \frac{\psi\left(\epsilon_{\mathrm{D}}\right) \epsilon_{\mathrm{D}}}{2 \kappa_{\nabla}}\right\} & \geq \min \left\{0.2 \frac{5 \sigma_{1}}{2 \kappa_{\nabla}} \epsilon_{\mathrm{P}} \epsilon_{\mathrm{D}}, \frac{\psi\left(\epsilon_{\mathrm{D}}\right) \epsilon_{\mathrm{D}}}{2 \kappa_{\nabla}}\right\} \\
& =\min \left\{\frac{\sigma_{1} \epsilon_{\mathrm{D}}}{2 \kappa_{\nabla}} \epsilon_{\mathrm{P}}, \frac{\sigma_{1} \epsilon_{\mathrm{D}}}{2 \kappa_{\nabla}} \epsilon_{\mathrm{D}}^{\sigma_{2}}\right\} \\
& =\frac{\sigma_{1} \epsilon_{\mathrm{D}}}{2 \kappa_{\nabla}} \min \left\{\epsilon_{\mathrm{P}}, \epsilon_{\mathrm{D}}^{\sigma_{2}}\right\} .
\end{aligned}
$$

Por (3.45) em (3.43), temos que

$$
\begin{aligned}
\kappa_{\Phi}\left[\frac{\epsilon_{\mathrm{P}}^{2}}{\min \left\{0.2 \rho \epsilon_{\mathrm{P}} \epsilon_{\mathrm{D}}, \frac{\psi\left(\epsilon_{\mathrm{D}}\right)}{2}, \frac{\psi\left(\epsilon_{\mathrm{D}}\right) \epsilon_{\mathrm{D}}}{2 \kappa_{\nabla}}\right\}^{\alpha}}\right] & \leq \kappa_{\Phi}\left[\frac{\epsilon_{\mathrm{P}}^{2}}{\left(\frac{\sigma_{1} \epsilon_{\mathrm{D}}}{2 \kappa_{\nabla}}\right)^{\alpha} \min \left\{\epsilon_{\mathrm{P}}, \epsilon_{\mathrm{D}}^{\sigma_{2}}\right\}^{\alpha}}\right] \\
& =\kappa_{\Phi}\left(\frac{2 \kappa_{\nabla}}{\sigma_{1}}\right)^{\alpha} \epsilon_{\mathrm{P}}^{2} \epsilon_{\mathrm{D}}^{-\alpha} \min \left\{\epsilon_{\mathrm{P}}, \epsilon_{\mathrm{D}}^{\sigma_{2}}\right\}^{-\alpha} .
\end{aligned}
$$


Como a quantidade de iterações é limitada por uma quantidade inteira, segue o resultado.

É importante notar que, analogamente ao que acontece na Hipótese 3.4, a constante $\kappa_{\Phi}$ em (3.42) depende apenas de $f, g$ e $h$ e dos parâmetros do algoritmo para minimização irrestrita empregado no Algoritmo FTARGET.

Temos agora todos os ingredientes para demonstrar o resultado principal deste capítulo: a complexidade de avaliação do Algoritmo FTARGET.

Teorema 3.7. Suponha que as Hipóteses 3.1, 3.2 e 3.4 valham e que $f(x) \leq f_{\max }$ para todo $x \in \mathcal{L}\left(\epsilon_{\mathrm{P}}^{2}\right)$. Então o Algoritmo FTARGET para no Passo F2 da Fase 1, ou existe $k \in\{0,1,2, \ldots\}$ tal que o Algoritmo FTARGET para na iteração $k$ da Fase 2 com $x^{\epsilon}$ satisfazendo os critérios do Passo T3 ou Passo T4. Mais ainda, o Algoritmo FTARget consome, na Fase 1, no máximo

$$
\left\lfloor\kappa_{V}\left[\frac{V\left(x^{-1}\right)}{\sigma_{1}^{\alpha}}\right] \epsilon_{\mathrm{D}}^{-\sigma_{2} \alpha}\right\rfloor
$$

avaliações de $g$ e $h$, e suas derivadas e, na Fase 2, no máximo

$$
\left\lfloor\left[\kappa_{\Phi}\left(\frac{2 \kappa_{\nabla}}{\sigma_{1}}\right)^{\alpha}\left(\frac{f_{\max }-f_{\min }}{0.1(1-\rho)}+1\right)\right] \epsilon_{\mathrm{P}} \epsilon_{\mathrm{D}}^{-\alpha} \min \left\{\epsilon_{\mathrm{D}}^{\sigma_{2}}, \epsilon_{\mathrm{P}}\right\}^{-\alpha}\right\rfloor
$$

avaliações de $f, g$ e $h$, e suas derivadas.

Demonstração. A primeira parte do teorema segue do Teorema 3.5, dado que a Hipótese 3.4 implica a Hipótese 3.3. O limitante (3.46) segue diretamente da Hipótese 3.4, dado que no Passo F1 a precisão $\varepsilon$ necessária para parada do algoritmo é $\psi\left(\epsilon_{\mathrm{D}}\right)=\sigma_{1} \epsilon_{\mathrm{D}}^{\sigma_{2}}$. O limitante $(3.47)$ é simplesmente uma cota superior do produto entre os limitantes (3.30) e (3.42), resultados respectivos dos Lemas 3.2 e 3.6 .

Novamente, vale ressaltar que a constante $\kappa_{V}$ em (3.46) depende apenas de $g, h$ e de parâmetros do algoritmo para minimização irrestrita empregado nos subproblemas do Algoritmo FTARGET, enquanto que, da mesma forma, a constante $\kappa_{\Phi}$ depende exclusivamente de $f, g, h$ e de parâmetros do algoritmo para minimização irrestrita. Note ainda que, por introduzir o limitante superior $f_{\max }$ para a função objetivo em $\mathcal{L}\left(\epsilon_{\mathrm{P}}^{2}\right)$, pudemos fazer com que o limitante de complexidade do Algoritmo FTARGET fosse independente de $x^{0}$, que não é nem uma entrada nem um dado do problema, mas um resultado de aplicar o algoritmo para minimização irrestrita do problema de minimizar $V(x)$ iniciando de $x^{-1}$ no Passo F1 do Algoritmo FTARget.

\subsection{Considerações sobre os resultados de complexidade}

Para explicitar um pouco mais os resultados decorrentes do Teorema 3.7, ilustramos na Tabela 3.1 os resultados obtidos neste capítulo para o caso em que

$$
\epsilon=\epsilon_{\mathrm{P}}=\epsilon_{\mathrm{D}}
$$




\begin{tabular}{|c|c|c|c|c|}
\hline & & \multicolumn{3}{|c|}{$\psi(\epsilon)=\sigma_{1} \epsilon^{\sigma_{2}}$} \\
\hline & & $\sigma_{2}=1$ & $\sigma_{2} \in(1,2)$ & $\sigma_{2}=2$ \\
\hline \multirow{2}{*}{\multicolumn{2}{|c|}{ 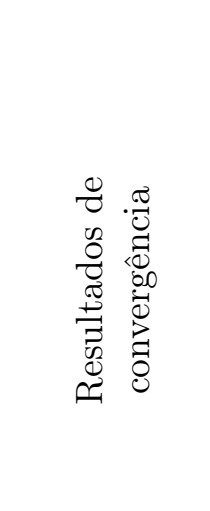 }} & \multicolumn{3}{|c|}{$\begin{array}{c}\text { Ponto estacionário aproximado inviável para } \epsilon \rightarrow 0 \text { (Fase } 1) \text {, } \\
\text { ponto AKKT (Fase } 2) \text {, ou } \ldots\end{array}$} \\
\hline & & $\begin{array}{l}\text { gradientes das restrições } \\
\text { não são } \xi \text {-uniformemente } \\
\text { positivamente linearmente } \\
\text { independentes com } \\
\xi=\sigma_{1} /(2 \sqrt{0,99})\end{array}$ & $\begin{array}{l}\text { MFCQ não vale } \\
\text { para } \epsilon \rightarrow 0\end{array}$ & $\begin{array}{l}\text { Łojasiewicz não vale } \\
\text { para } \epsilon \rightarrow 0\end{array}$ \\
\hline \multirow{3}{*}{ 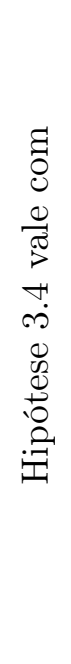 } & $\begin{array}{ll}\sim & \overparen{1} \\
\| & \| \\
0 & g\end{array}$ & $\begin{array}{l}\text { Fase 1: } O\left(\epsilon^{-2}\right) \\
\text { Fase 2: } O\left(\epsilon^{-3}\right)\end{array}$ & $\begin{array}{l}\text { Fase 1: } O\left(\epsilon^{-2 \sigma_{2}}\right) \\
\text { Fase 2: } O\left(\epsilon^{-1-2 \sigma_{2}}\right)\end{array}$ & $\begin{array}{l}\text { Fase 1: } O\left(\epsilon^{-4}\right) \\
\text { Fase 2: } O\left(\epsilon^{-5}\right)\end{array}$ \\
\hline & 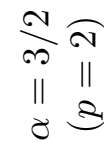 & $\begin{array}{l}\text { Fase 1: } O\left(\epsilon^{-1.5}\right) \\
\text { Fase 2: } O\left(\epsilon^{-2}\right)\end{array}$ & $\begin{array}{l}\text { Fase 1: } O\left(\epsilon^{-1.5 \sigma_{2}}\right) \\
\text { Fase 2: } O\left(\epsilon^{-0.5-1.5 \sigma_{2}}\right)\end{array}$ & $\begin{array}{l}\text { Fase 1: } O\left(\epsilon^{-3}\right) \\
\text { Fase 2: } O\left(\epsilon^{-3.5}\right)\end{array}$ \\
\hline & 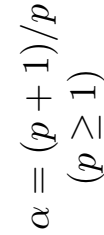 & $\begin{array}{l}\text { Fase 1: } O\left(\epsilon^{-\frac{(p+1)}{p}}\right) \\
\text { Fase 2: } O\left(\epsilon^{1-2 \frac{(p+1)}{p}}\right)\end{array}$ & $\begin{array}{l}\text { Fase 1: } O\left(\epsilon^{-\sigma_{2} \frac{(p+1)}{p}}\right) \\
\text { Fase 2: } O\left(\epsilon^{1-\left(1+\sigma_{2}\right) \frac{(p}{2}}\right.\end{array}$ & $\begin{array}{l}\text { Fase 1: } O\left(\epsilon^{-2 \frac{(p+1)}{p}}\right) \\
\text { Fase 2: } O\left(\epsilon^{1-3 \frac{(p+1)}{p}}\right)\end{array}$ \\
\hline
\end{tabular}

Tabela 3.1: Resumo dos resultados de convergência e complexidade para escolhas diferentes do algoritmo para minimização irrestrita e para a função $\psi(\epsilon)=\sigma_{1} \epsilon^{\sigma_{2}}$ com $\sigma_{1}>0$ e para escolhas diferentes de $\sigma_{2} \in[1,2]$.

é suficientemente pequeno e para diferentes escolhas do algoritmo para minimização irrestrita à luz da Hipótese 3.4 e diferentes valores de $\sigma_{2}$ para funções $\psi$ da forma (3.20).

Em primeiro lugar, considerando (3.48), a complexidade da Fase 1 do Algoritmo FTARGET, por (3.46), se reduz a

$$
O\left(\epsilon^{-\sigma_{2} \alpha}\right)
$$

enquanto que, de (3.47), a complexidade da Fase 2 do Algoritmo FTARGET é dada por

$$
O\left(\epsilon^{1-\left(1+\sigma_{2}\right) \alpha}\right) \text {. }
$$

Note que, da definição (3.26), $\omega=1$, o que faz nossa discussão da Seção 3.2.2 válida. A escolha (3.48) foi feita de maneira arbitrária por ser conveniente à nossa discussão aqui, certamente 
diferente escolhas resultarão em variações dos resultados expostos na Tabela 3.1. Por exemplo, a alternativa $\epsilon_{\mathrm{D}}=\epsilon_{\mathrm{P}}^{2 / 3}$ é sugerida em [26], resultando em um limitante de complexidade de $O\left(\epsilon_{\mathrm{P}}^{-3 / 2}\right)$ para o caso em que $p=2, \sigma_{2}=1$ e $\alpha=3 / 2$, mas implicando que $\omega=\infty$. Em particular, ao considerar (3.48) estamos implicitamente assumindo que o escalamento do problema original é razoável. Note ainda que a complexidade da Fase 1 exposta na Tabela 3.1 é apenas informativa, haja visto que a complexidade da Fase 2 domina a da Fase 1 e é a complexidade geral do Algoritmo FTARGET.

Com $\sigma_{2}=2$, nós obtemos o "melhor resultado" no sentido de que o Algoritmo FTARGET para apenas quando encontra um ponto AKKT (não escalado) no limite ou quando uma propriedade muito fraca - a desigualdade de Łojasiewicz - não vale no limite. Com $\sigma_{2}=1$, o Algoritmo FTArget para quando um ponto AKKT é encontrado ou quando, no limite, não vale uma condição relaxada de Mangasarian-Fromovitz com tolerância $\xi=\sigma_{1} /(2 \sqrt{0,99})$. Entre os extremos $1<\sigma_{2}<2$, ou o algoritmo converge a um ponto AKKT ou MFCQ não vale no limite. Como era de se esperar, a melhor complexidade acontece com $\sigma_{1}=1$ e a pior com $\sigma_{2}=2$. Também como era de se esperar, a complexidade geral do Algoritmo FTARgET melhora se a Hipótese 3.4 for satisfeita com $\alpha$ mais próximo possível de um, já que, neste caso, $p$ cresce.

Agora, compararemos os resultados obtidos aqui com aqueles obtidos em [23, 26, 28] para $p=1 \mathrm{e}$ $p=2$, focando apenas no caso em que $\epsilon=\epsilon_{\mathrm{P}}=\epsilon_{\mathrm{D}}$. Nestes trabalhos, a complexidade para convergir a um ponto que satisfaz as condições KKT escaladas é considerado, enquanto que nos resultados obtidos no presente trabalho analisamos a complexidade para obter um ponto AKKT não escalado. Por KKT escalado denotamos que (3.10) e (3.12) são escalados de forma que valha, para uma tripla aproximada de primeira ordem $(x, \lambda, \mu)$,

$$
\begin{aligned}
\sqrt{V(x)} & \leq \epsilon \\
\left\|\nabla f(x)+\sum_{i=1}^{m} \lambda_{i} \nabla h_{i}(x)+\sum_{i=1}^{q} \mu_{j} \nabla g_{j}(x)\right\| & \leq \epsilon\|(1, \lambda, \mu)\| \\
\sum_{j=1}^{q} \mu_{j} g_{j}(x) & \leq 2 \epsilon\|(1, \lambda, \mu)\|,
\end{aligned}
$$

onde $\mu_{j} \geq 0$ para $j=1, \ldots, q$.

O que se mostra em $[23,28]$ é que se $f, g$ e $h$ são funções continuamente diferenciáveis com gradiente Lipschitz contínuos, então uma tripla $(x, \lambda, \mu)$ que satisfaça (3.49) ou (3.5)/(3.8) pode ser encontrada em, no máximo,

$$
\left\lfloor\kappa_{1} \frac{\sqrt{V\left(x^{-1}\right)}+f_{\max }-f_{\min }}{\epsilon^{2}}+\kappa_{2}|\log \epsilon|+\kappa_{3}\right\rfloor
$$

avaliações de $f, g$ e $h$ e suas derivadas primeiras, onde $\kappa_{1}, \kappa_{2}$ e $\kappa_{3}$ são constantes independentes de $\epsilon$. Esse resultado é uma ordem melhor que o limitante $O\left(\epsilon^{-3}\right)$ presente na Tabela 3.1 para o caso $\alpha=2$ e $\sigma_{2}=1$, o que indica que alcançar as condições KKT escaladas pode ser mais fácil que convergir a um ponto AKKT não escalado usando apenas derivadas primeiras. Focamos no caso em que $\sigma_{2}=1$ porque as implicações em termos de degenerescência para $\epsilon \rightarrow 0$ não são discutidas em [23, 28], o que não deixa claro se os algoritmos que estão sendo comparados declaram falha ao satisfazer KKT escalado ou não escalado sob as mesmas circunstâncias.

Por outro lado, a comparação é mais complicada se admitirmos o uso de derivadas primeiras e segundas, porque os resultados em [26] são expostos usando-se uma medida diferente de criticalidade $\chi(x)$, cujo valor é o descenso maximal que é alcançável sobre a função linearizada na interseção de uma esfera unitária e o conjunto viável definido pelas restrições de positividade das variáveis de folga usada para as desigualdades (veja [26] para mais detalhes). Essa medida de criticalidade é também 
usada na forma escalada, no sentido de que ela deve estar por baixo do valor $\epsilon\|(1, \lambda, \mu)\|$ quando aplicada ao Lagrangiano (no espírito de (3.49)), ou abaixo de $\epsilon$ quando aplicada à raiz quadrada da viabilidade. Esta última condição é conceitualmente similar a (3.5)/(3.8) mas é mais forte porque envolve o gradiente da raiz quadrada da viabilidade, ao invés do gradiente da viabilidade somente; portanto, hipóteses mais fortes nos valores singulares dos Jacobianos das restrições são necessárias para assegurar sua equivalência. Neste contexto, demonstra-se que, se $f, g$ e $h$ são duas vezes continuamente diferenciáveis com gradientes e Hessianas Lipschitz contínuos, e se uma hipótese acerca da solução do subproblema vale (veja [26] para mais detalhes), então uma tripla $(x, \lambda, \mu)$ satisfazendo essas condições de criticalidade escaladas alternativas podem ser obtidas em no máximo

$$
\left\lfloor\kappa_{1} \frac{\sqrt{V\left(x^{-1}\right)}+f_{\max }-f_{\min }}{\epsilon^{2}}+\kappa_{2}\right\rfloor
$$

avaliações de $f, g$ e $h$, e suas derivadas primeiras e segundas, onde $\kappa_{1}$ e $\kappa_{2}$ são independentes de $\epsilon$. Comparado ao caso em que $p=1$, este limitante é agora (em termos assintóticos) idêntico ao resultados correspondente na Tabela 3.1, onde $\alpha=3 / 2$ e $\sigma_{2}=1$. 


\section{Capítulo 4}

\section{Implementação e experimentos numéricos}

Para esta fase de implementação e experimentação numérica, selecionamos os dois algoritmos para minimização irrestrita que usam modelos de ordem superior, o $\mathrm{AR}_{p}$ e o $\mathrm{AR}_{p}^{s}$, descritos nas Seções 2.2.2 e 2.2.3 do Capítulo 2. Desenvolvemos o trabalho deste capítulo em duas etapas.

Na primeira etapa, o interesse inicial era experimentar o $\mathrm{AR}_{p}$ com derivadas terceiras, portanto, denotamos por $\mathrm{AR}_{3}$. Começamos os experimentos com uma implementação em Matlab ${ }^{\circledR}$ para minimizar polinômios. Numa primeira instância, nosso interesse era calcular a direção $d^{k}$ pela minimização global de $m_{3}\left(x^{k}, d, \sigma_{k}\right)$. Para isso fizemos uso de dois algoritmos publicados recentemente na literatura, que detalhamos nas Seções 4.1.1 e 4.1.2. Entretanto, nesta primeira etapa, não obtivemos muito sucesso, mas mantemos aqui o registro do nosso trabalho, descrito com riqueza de detalhes nas Seções 4.1, 4.2 e 4.3.

Com isso começamos a segunda etapa, que consistiu em trabalhar no algoritmo $\mathrm{AR}_{p}^{s}$. Nesta etapa, estudamos e implementamos o $\mathrm{AR}_{p}^{s}$ usando derivadas segundas e terceiras. Os subproblemas foram resolvidos pela minimização aproximada de $m_{p}\left(x^{k}, d, \sigma_{k, j}\right)$. Mostrando-se como um algoritmo promissor, prosseguimos com os experimentos, estendendo-os a problemas clássicos da literatura, e mostrando a potencialidade do algoritmo em um problema de grande porte. Descrevemos esta etapa, com detalhes, nas Seções 4.4 e 4.5 .

\subsection{Implementação computacional: $\mathrm{AR}_{3}$}

Nesta seção, discorreremos sobre a implementação computacional do $\mathrm{AR}_{p}$ para $p=3$, ou seja, a cada iteração do Algoritmo 2.2 encontramos o passo $d^{k}$ resolvendo aproximadamente o problema

$$
\min _{d \in \mathbb{R}^{n}} m_{3}\left(x^{k}, d, \sigma_{k}\right)=f\left(x^{k}\right)+\nabla f\left(x^{k}\right) d+\frac{1}{2} \nabla^{2} f\left(x^{k}\right) d^{2}+\frac{1}{6} \nabla^{3} f\left(x^{k}\right) d^{3}+\frac{\sigma_{k}}{4}\|d\|^{4}
$$

A minimização aproximada de $m_{3}\left(x^{k}, d, \sigma_{k}\right)$ consiste no passo mais complexo do Algoritmo 2.2. Além disso, é o único passo que carece de detalhes de implementação. Por se tratar de um problema irrestrito, há inúmeros métodos que poderíamos empregar para sua resolução. Todavia, temos que 
$m_{3}$ é um polinômio de grau quatro de $n$ variáveis $d_{1}, d_{2}, \ldots, d_{n}$, haja visto que

$$
\begin{aligned}
m_{3}\left(x^{k}, d, \sigma_{k}\right)= & f\left(x^{k}\right)+\sum_{i=1}^{n}\left[f\left(x^{k}\right)\right]_{i} d_{i}+\frac{1}{2} \sum_{i, j}^{n}\left[\nabla^{2} f\left(x^{k}\right)\right]_{i, j} d_{i} d_{j} \\
& +\frac{1}{6} \sum_{i, j, k}^{n}\left[\nabla^{3} f\left(x^{k}\right)\right]_{i, j, k} d_{i} d_{j} d_{k}+\frac{\sigma_{k}}{4}\left(\sum_{i=1}^{n} d_{i}^{2}\right)^{2} .
\end{aligned}
$$

Notemos que para $\|d\|$ tendendo ao infinito, o termo $\|d\|^{4}$ cresce mais rapidamente que os demais. Como $\frac{\sigma_{k}}{4}\|d\|^{4}$ é sempre não negativo para todo $d$, então, para $\|d\| \rightarrow \infty, m_{3}\left(x^{k}, d, \sigma_{k}\right)$ tende a infinito, o que implica que $m_{3}$ é limitado inferiormente e, portanto, possui minimizador global.

Desta forma, tentamos explorar estas características dos subproblemas. Iniciamos nossas tentativas de implementação com o objetivo de minimizar globalmente o modelo $m_{3}\left(x^{k}, d, \sigma_{k}\right)$. Começamos com o artigo de Qi, Wan e Yang [60], em que os autores propõem um algoritmo para minimização global de polinômios quárticos normais, ou seja, polinômios multivariados de grau 4 cujo coeficiente do termo de grau 4 é um tensor definido positivo (daremos mais detalhes sobre este método na Seção 4.1.1 adiante). Uma adaptação deste método será descrita com riqueza de detalhes na Seção 4.1.1. Não obstante, em linhas gerais, o método consiste em minimizar o polinômio $P(x)$ usando um método de Newton globalizado para minimização irrestrita até convergir a um ponto aproximadamente estacionário de primeira ordem do problema. Tendo convergido a um tal ponto $x^{*}$, os autores propõem ferramentas para verificar se este ponto é um maximizador local, ponto de sela ou minimizador local. Nos casos em que o ponto for maximizador local ou ponto de sela, a estratégia é usar informações de segunda ordem e tomar uma direção de curvatura negativa da Hessiana de $P$ como passo para continuar a minimização. Caso $x^{*}$ seja um minimizador local, os autores propõem encontrar um ponto viável $\bar{x}$ para o sistema não linear

$$
\left\{\begin{array}{cc}
\nabla P(x) & =0 \\
P(x)-x_{n+1} & =0 \\
x_{n+1}-P\left(x^{*}\right)+\epsilon & \leq 0
\end{array}\right.
$$

para $\epsilon>0$. Se $\bar{x}$ não existir, $x^{*}$ é minimizador $\epsilon$-global de $P(x)$, caso contrário, $\bar{x}-x^{*}$ é uma possível direção de saída de $x^{*}$ ao longo da qual certamente existe um ponto em que $P$ vale menos, a saber, $\bar{x}$. Todavia, optamos por não usá-lo na prática, haja visto que experimentos numéricos que fizemos mostraram que o algoritmo proposto no artigo não encontra a solução global dos polinômios (veja Seção 4.2 adiante). Isso já é de se esperar, pois, devido a limitações computacionais, não existe método numérico que ateste com certeza para qualquer polinômio $P$ e qualquer ponto $x^{*}$ que o sistema não linear (4.2) é viável ou não. Além disso, se fossemos capazes de garantidamente encontrar um ponto viável para o sistema não linear (4.2) para qualquer $P$ e qualquer $x^{*}$, bastaria definir um método que, a cada iteração, encontre um tal ponto viável. É trivial ver que, quando não conseguíssemos mais encontrar um tal ponto, é porque estaríamos em um minimizador global.

Ainda com a ideia de minimizar globalmente o modelo, encontramos um método chamado Gloptipoly $[46,47]$ que, pela resolução de um problema de programação semidefinida, pode encontrar o minimizador global do problema de minimizar um polinômio qualquer. Por estar implementado em Matlab ${ }^{\circledR}$ fizemos uma implementação de $\mathrm{AR}_{3}$ em Matlab ${ }^{\circledR} \mathrm{R} 2015 \mathrm{a}$, que denotaremos por $\mathbf{A R}_{3}^{P}$.

Por outro lado, já que a condição (2.29) do Passo 2 do Algoritmo 2.2 pode ser alcançada pela minimização aproximada do modelo, fizemos uma implementação de $\mathrm{AR}_{3}$ que usa GENCAN [3, 12,13] para resolver os subproblemas de minimizar aproximadamente o modelo. Esta versão foi implementada em Fortran 2008 e usa Algencan 3.1.0 ${ }^{1}$ [14]. A esta implementação chamaremos de $\mathbf{A R}_{3}^{G}$.

\footnotetext{
${ }^{1}$ Esta é ainda uma versão beta. A última versão estável de Algencan está disponível para download em http: //www.ime.usp.br/ egbirgin/tango/downloads.php.
} 
Na seção a seguir, apresentaremos uma adaptação do método proposto em [60]. Neste, procuramos transformar o que eles apresentaram como determinístico em heurístico, para procurar, de alguma forma, sair do minimizador local do problema. Logo em seguida, daremos uma descrição resumida do método Gloptipoly e, depois, mostraremos um breve experimento numérico, em que Gloptipoly obtém resultados melhores que os encontrados nos experimentos realizados em [60] atestando, por conseguinte, que o método proposto não minimiza globalmente os polinômios. Finalmente, apresentaremos os resultados dos experimentos realizados com as implementações de $\mathrm{AR}_{3}$.

\subsubsection{Um algoritmo para minimização de polinômios quárticos normais}

Nesta seção, apresentaremos um método para minimização de polinômios quárticos normais baseado no trabalho de Qi, Wan e Yang [60]. Neste artigo, os autores propõem um algoritmo que garante minimização global de tais polinômios, porém que exige a resolução de subproblemas difíceis e que podem não possuir solução numericamente computável em tempo hábil, portanto, não é um algoritmo prático. Como eles não nomeiam o método deles, chamaremos de GMNQP. Nossa abordagem aqui consiste em detalhar as demonstrações de [60], além de propor um método que, embora não garanta a minimização global de polinômios quárticos normais, garante ao menos convergência a um minimizador local, e não apenas a um ponto crítico como métodos clássicos para programação não linear.

Seja $T$ um tensor de ordem $p$, cujos elementos são denotados por

$$
T_{i_{1}, i_{2}, \ldots, i_{p}}, \operatorname{com} i_{1}, i_{2}, \ldots, i_{p}=1,2, \ldots, n .
$$

Dado $x \in \mathbb{R}^{n}$, consideraremos

$$
T x^{p}=\sum_{i_{1}, i_{2}, \ldots, i_{p}=1}^{n} T_{i_{1}, i_{2}, \ldots, i_{p}} x_{i_{1}} x_{i_{2}} \ldots x_{i_{p}},
$$

$\left(T x^{p}\right) \in \mathbb{R}$, como sendo $T$ aplicado $p$ vezes a $x$.

Dizemos que $T$ é definido positivo se $T x^{p}>0$, para todo $x \in \mathbb{R}^{n}$ tal que $\|x\|=1$.

Sejam $T_{i}$, com $i=1,2,3$ e 4, tensores simétricos de ordem $i$ e $t_{0} \in \mathbb{R}$. Consideraremos um polinômio quártico a função

$$
P(x)=T_{4} x^{4}+T_{3} x^{3}+T_{2} x^{2}+T_{1} x+t_{0}
$$

Dizemos que $P(x)$ é um polinômio quártico normal se $T_{4}$ for definido positivo.

Notemos que, no caso de nosso polinômio definido pelo modelo $m_{3}$ em (4.1), computar $T_{4} x^{4}$ equivale a calcular $\frac{\sigma_{k}}{4}\|x\|^{4}$. Como $\sigma_{k} \geq \sigma_{\min }>0, \frac{\sigma_{k}}{4}\|x\|^{4}>0$ para todo $x \in \mathbb{R}^{n}$ tal que $\|x\|=1$. Por conseguinte, $m_{3}$ definido em (4.1) é um polinômio quártico normal.

Consideremos então $P$, como definido em (4.3), um polinômio quártico normal. Estamos interessados no problema

$$
\underset{x \in \mathbb{R}^{n}}{\operatorname{Minimizar}} P(x)
$$


Dizemos que $x$ é ponto crítico de $P$ se $\nabla P(x)=0$.

Seja $s \in \mathbb{R}^{n}$ e $\alpha \in \mathbb{R}$. Pelo Teorema de Taylor, existe $\bar{x} \in \mathbb{R}^{n}, \bar{x}=x+\bar{\alpha} p$, com $\bar{\alpha}$ entre 0 e $\alpha$ (i.e. $0<\bar{\alpha}<\alpha$ ou $\alpha<\bar{\alpha}<0$ ), tal que

$$
P(x+\alpha s)=P(x)+\alpha \nabla P(x) s+\frac{1}{2} \alpha^{2} \nabla^{2} P(x) s^{2}+\frac{1}{6} \alpha^{3} \nabla^{3} P(x) s^{3}+\frac{1}{24} \alpha^{4} \nabla^{4} P(\bar{x}) s^{4} .
$$

Consideremos o teorema a seguir, extraído de [60, Proposição 2]. Este teorema estabelece a característica de um ponto crítico de $P$ pela observação de suas derivadas de ordem dois e três. Reescrevemos a demonstração por fins de detalhamento dos resultados.

Teorema 4.1. Seja $P: \mathbb{R}^{n} \rightarrow \mathbb{R}$ um polinômio quártico normal, como definido em (4.3). Consideremos $\hat{x} \in \mathbb{R}^{n}$ um ponto crítico de P. Então,

(a) $S e \nabla^{2} P(\hat{x})$ for definida negativa, então $\hat{x}$ é um maximizador local de $P$.

(b) $S e \nabla^{2} P(\hat{x})$ for definida positiva, ou se for semidefinida positiva e

$$
\nabla^{3} P(\hat{x}) s^{3}=0
$$

para todo $s \in \mathbb{R}^{n}$ tal que $\nabla^{2} P(\hat{x}) s^{2}=0$, então $\hat{x}$ é um minimizador local de $P$.

(c) Caso contrário, $\hat{x}$ é um ponto de sela de $P$.

Demonstração. Notemos que, por (4.5), $P(\hat{x}+\alpha s)-P(\hat{x})$ possui o mesmo sinal que $\nabla^{2} P(\hat{x}) s^{2}$ para um $s \in \mathbb{R}^{n}$ fixo e valores de $\alpha$ suficientemente pequenos, em módulo. Neste contexto, analisaremos quatro casos:

(i) $\nabla^{2} P(\hat{x})$ é indefinida,

(ii) $\nabla^{2} P(\hat{x})$ é negativa definida,

(iii) $\nabla^{2} P(\hat{x})$ é definida positiva e

(iv) $\nabla^{2} P(\hat{x})$ é semidefinida positiva ou negativa.

Para o caso (i), existem direções $s \in \mathbb{R}^{n}$ tais que $\nabla^{2} P(\hat{x}) s^{2}<0$, em cujo caso $P(\hat{x}+\alpha s)-$ $P(\hat{x})<0$ para todo $\alpha \in \mathbb{R}$ suficientemente pequeno, em módulo, e $\nabla^{2} P(\hat{x}) s^{2}>0$, em cujo caso $P(\hat{x}+\alpha s)-P(\hat{x})>0$ para todo $\alpha \in \mathbb{R}$ suficientemente pequeno em módulo. Portanto, $\hat{x}$ é um ponto de sela de $P$.

Para o caso (ii), temos que $\nabla^{2} P(\hat{x}) s^{2}<0$ para todo $s \in \mathbb{R}^{n}$. Portanto, $P(\hat{x}+\alpha s)-P(\hat{x})<$ 0 para todo $s \in \mathbb{R}^{n}$ e para todo $\alpha \in \mathbb{R}$ suficientemente pequeno, em módulo. Logo, $\hat{x}$ é maximizador local de $P$.

Para o caso (iii), temos que $\nabla^{2} P(\hat{x}) s^{2}>0$ para todo $s \in \mathbb{R}^{n}$. Portanto, $P(\hat{x}+\alpha s)-P(\hat{x})>0$ para todo $s \in \mathbb{R}^{n}$ e todo $\alpha \in \mathbb{R}$ suficientemente pequeno, em módulo. Logo, $\hat{x}$ é minimizador local de $P$.

Para o caso (iv), por sua vez, consideremos

$$
\mathcal{N}=\left\{s \in \mathbb{R}^{n} \mid \nabla^{2} P(\hat{x}) s^{2}=0\right\}
$$

Notemos que $\mathcal{N}$ contém ao menos um vetor não nulo. 
Consideremos, em primeiro lugar, que $\nabla^{2} P(\hat{x})$ é semidefinida positiva. Por um lado, para todo $s \notin \mathcal{N}, \nabla^{2} P(\hat{x}) s^{2}>0$, o que implica que, para $\alpha \in \mathbb{R}$ suficientemente pequeno, em módulo,

$$
P(\hat{x}+\alpha s)-P(\hat{x})>0 .
$$

Por outro lado, para todo $s \in \mathcal{N}$, consideremos dois casos:

1. se $\nabla^{3} P(\hat{x}) s^{3}=0$, então, por (4.5),

$$
P(\hat{x}+\alpha s)-P(\hat{x})=\frac{1}{24} \alpha^{4} \nabla^{4} P(\bar{x}) s^{4},
$$

para algum $\bar{x}$. Como $\nabla^{4} P(x)=24 T_{4}$ e $P$, definido em (4.3), é normal, temos que $P(\hat{x}+\alpha s)-P(\hat{x})>0$. Isso, junto com (4.6), implica que $\hat{x}$ é minimizador local de $P$.

2. se $\nabla^{3} P(\hat{x}) s^{3} \neq 0$, então, por (4.5), o sinal de $P(\hat{x}+\alpha s)-P(\hat{x})$ coincide com o sinal de $\alpha^{3} \nabla^{3} P(\hat{x}) s^{3}$ para valores de $\alpha$ suficientemente pequenos, em módulo, que, por sua vez, muda de sinal conforme $\alpha$ muda de sinal. Isso implica que $\hat{x}$ é ponto de sela de $P$.

Consideremos, em segundo lugar, que $\nabla^{2} P(\hat{x})$ é semidefinida negativa. Por um lado, para todo $s \notin \mathcal{N}, \nabla^{2} P(\hat{x}) s^{2}<0$, o que implica que, para $\alpha \in \mathbb{R}$ suficientemente pequeno, em módulo,

$$
P(\hat{x}+\alpha s)-P(\hat{x})<0 .
$$

Por outro lado, para todo $s \in \mathcal{N}$, consideremos dois casos:

1. se $\nabla^{3} P(\hat{x}) s^{3}=0$, então, por $(4.5)$,

$$
P(\hat{x}+\alpha s)-P(\hat{x})=\frac{1}{24} \alpha^{4} \nabla^{4} P(\bar{x}) s^{4},
$$

para algum $\bar{x}$. Como $\nabla^{4} P(x)=24 T_{4}$ e $P$, definido em (4.3), é normal, temos que $P(\hat{x}+\alpha s)-P(\hat{x})>0$. Isso, junto com (4.7), implica que $\hat{x}$ é ponto de sela de $P$.

2. se $\nabla^{3} P(\hat{x}) s^{3} \neq 0$, então, por (4.5), o sinal de $P(\hat{x}+\alpha s)-P(\hat{x})$ coincide com o sinal de $\alpha^{3} \nabla^{3} P(\hat{x}) s^{3}$ para valores de $\alpha$ suficientemente pequenos, em módulo, que, por sua vez, muda de sinal conforme $\alpha$ muda de sinal. Isso implica que $\hat{x}$ é ponto de sela de $P$.

Segue o resultado.

Bem como em algoritmos gerais de otimização, o método que apresentaremos consiste em procurar por pontos críticos de $P$ usando estratégias clássicas de descida. A diferença fundamental é que aqui, ao invés de pararmos em pontos críticos de $P$, procuraremos sair de tais pontos críticos usando os resultados do Teorema 4.1 e direções de tipo tunneling.

Dizemos que $s \in \mathbb{R}^{n}$ é uma direção do tipo tunneling para $P$ se existir algum $\alpha \in \mathbb{R}$ tal que

$$
P(x+\alpha s)<P(x) .
$$


Neste contexto, é essencial saber determinar se $s$ é uma direção do tipo tunneling. Dados $x, s \in \mathbb{R}^{n}$, consideraremos a notação

$$
\begin{aligned}
& a(x, s) \stackrel{\text { def }}{=} \frac{1}{24} \nabla^{4} P(x) s^{4}, \\
& b(x, s) \stackrel{\text { def }}{=}-\frac{1}{6} \nabla^{3} P(x) s^{3}, \\
& c(x, s) \stackrel{\text { def }}{=} \frac{1}{2} \nabla^{2} P(x) s^{2}, \\
& d(x, s) \stackrel{\text { def }}{=}-\nabla P(x) s .
\end{aligned}
$$

Definimos de (4.5) a função

$$
\phi_{x, s}(\alpha) \stackrel{\text { def }}{=} P(x+\alpha s)-P(x)=a(x, s) \alpha^{4}-b(x, s) \alpha^{3}+c(x, s) \alpha^{2}-d(x, s) \alpha .
$$

Notemos que, como $a(x, s)>0$, para todo $x, s \in \mathbb{R}^{n}, \phi_{x, s}(\cdot)$ tende a infinito quando $\alpha$ tende a mais ou menos infinito. Portanto, $\phi_{x, s}(\cdot)$ possui minimizador global.

Consideremos o caso em que estamos em um ponto crítico de $P$. Assim sendo, sejam $\hat{x} \in \mathbb{R}^{n}$ um ponto crítico de $P, s \in \mathbb{R}^{n}$ e $\alpha \in \mathbb{R}$. Como $\hat{x}$ é ponto crítico de $P, d(\hat{x}, s)=0$ para todo $s \in \mathbb{R}^{n}$ e, portanto,

$$
\phi_{\hat{x}, s}(\alpha)=\alpha^{2}\left[a(\hat{x}, s) \alpha^{2}-b(\hat{x}, s) \alpha+c(\hat{x}, s)\right] \stackrel{\text { def }}{=} \alpha^{2} \omega_{\hat{x}, s}(\alpha) .
$$

Uma direção $s$ é do tipo tunneling a partir de $\hat{x}$ se, e somente se, existe um escalar $\alpha$ não nulo tal que $\phi_{\hat{x}, s}(\alpha)<0$. Como $\alpha^{2} \geq 0$ para todo $\alpha \in \mathbb{R}$, então existe $\alpha \in \mathbb{R}$ tal que $\phi_{\hat{x}, s}(\alpha)<0$ se, e somente se, existe $\alpha \in \mathbb{R}$ tal que $\omega_{\hat{x}, s}(\alpha)<0$. Como $P$ é um polinômio quártico normal e $\nabla^{4} P(\hat{x})=24 T_{4}$, então $a(\hat{x}, s)$ como definido em (4.8) é positivo para todo $s \in \mathbb{R}^{n}$. Por conseguinte, $\omega_{\hat{x}, s}(\alpha)$ é uma parábola convexa, donde segue que existe $\alpha \in \mathbb{R}$ tal que $\omega_{\hat{x}, s}(\alpha)<0$ se, e somente se, $\omega_{\hat{x}, s}(\alpha)$ possui duas raízes reais distintas, o que acontece se, e somente se, seu discriminante for positivo, ou seja, se, e somente se

$$
\Delta(\hat{x}, s) \stackrel{\text { def }}{=} b(\hat{x}, s)^{2}-4 a(\hat{x}, s) c(\hat{x}, s)>0
$$

Para encontrar uma direção do tipo tunneling usando o resultado do Teorema 4.1, computamos o menor autovalor $\hat{\lambda}$ de $\nabla^{2} P(\hat{x})$.

- Se $\hat{\lambda}<0$ calculamos o autovetor $\hat{s}$ associado a $\hat{\lambda}$. Por (4.8), $a(\hat{x}, \hat{s})>0$, e por (4.10), $c(\hat{x}, \hat{s})<0$, donde segue, de (4.14), que $\Delta(\hat{x}, \hat{s})>0$. Portanto, $\hat{s}$ é uma direção do tipo tunneling de $P$ a partir de $\hat{x}$.

- Se $\hat{\lambda}=0$ e existe um autovetor $\hat{s}$ associado a $\hat{\lambda}$ tal que $\nabla^{3} P(\hat{x}) \hat{s}^{3} \neq 0$, por $(4.9), b(\hat{x}, \hat{s}) \neq 0$ e, por (4.10), $c(\hat{x}, \hat{s})=0$, donde segue, de (4.14), que $\Delta(\hat{x}, \hat{s})>0$. Portanto, $\hat{s}$ é uma direção do tipo tunneling de $P$ a partir de $\hat{x}$.

- Se $\hat{\lambda}>0$, então $\nabla^{2} P(\hat{x})$ é definida positiva e estamos num minimizador local de $P$. Este é o caso mais difícil, pois não somos capazes de conseguir alguma direção oriunda de informações de segunda ordem ao longo da qual $P$ decresça. Neste caso, consideraremos dois procedimentos heurísticos:

1. sortearemos vetores aleatórios $\left\{v_{1}, \ldots, v_{\ell}\right\}$ e testaremos se algum deles é uma direção do tipo tunneling, isto é, se $\Delta\left(\hat{x}, v_{i}\right)>0$, para algum $i=1,2, \ldots, \ell$. 
2. tentaremos encontrar, usando algum método para resolução de sistemas não lineares, um ponto $\left(\bar{x}, \bar{x}_{n+1}\right)$ viável para o sistema não linear

$$
\left\{\begin{array}{cl}
\nabla P(x) & =0 \\
P(x)-x_{n+1} & =0 \\
x_{n+1}-P(\hat{x})+\epsilon & \leq 0
\end{array}\right.
$$

para um dado $\epsilon>0$. Se existir $\bar{x}$ viável para (4.15), então a desigualdade junto com a segunda equação implica que $P(\bar{x}) \leq P(\hat{x})-\epsilon$, ou seja, que ao longo da direção $\bar{x}-\hat{x}$ existe um ponto, especificamente $\bar{x}$, em que $P$ vale menos que no minimizador local $\hat{x}$.

Se não conseguirmos encontrar uma direção do tipo tunneling a partir de $\hat{x}$, então terminamos a execução do algoritmo. Caso contrário, tendo encontrado uma direção do tipo tunneling $\hat{s}$ a partir de $\hat{x}$, computamos o tamanho $\hat{\alpha}$ do passo como minimizador global de $\phi_{\hat{x}, \hat{s}}(\cdot)$. Como $\hat{x}$ é um ponto crítico de $\phi_{x, p}(\cdot)$, sua derivada primeira é dada por

$$
\phi_{x, s}^{\prime}(\alpha)=4 a(x, s) \alpha^{3}-3 b(x, s) \alpha^{2}+2 c(x, s) \alpha=\alpha\left[4 a(x, s) \alpha^{2}-3 b(x, s) \alpha+2 c(x, s)\right] .
$$

Por conseguinte, os pontos de críticos de $\phi_{\hat{x}, \hat{s}}(\cdot)$ são dados pelas raízes de $\phi_{\hat{x}, \hat{s}}^{\prime}(\cdot)$, ou seja,

$$
\alpha_{0}=0, \quad \alpha_{1}=\frac{3 b(\hat{x}, \hat{s})+\sqrt{9 b(\hat{x}, \hat{s})^{2}-32 a(\hat{x}, \hat{s}) c(\hat{x}, \hat{s})}}{8 a(\hat{x}, \hat{s})} \quad \text { e } \quad \alpha_{2}=\frac{3 b(\hat{x}, \hat{s})-\sqrt{9 b(\hat{x}, \hat{s})^{2}-32 a(\hat{x}, \hat{s}) c(\hat{x}, \hat{s})}}{8 a(\hat{x}, \hat{s})} .
$$

Seria possível encontrar o minimizador global de $\phi_{\hat{x}, \hat{s}}(\cdot)$ apenas avaliando-a nos pontos dados em (4.16) e tomando aquele cujo valor funcional seja o menor. Todavia, é possível determinar o minimizador global de $\phi_{\hat{x}, \hat{s}}$ apenas analisando o sinal de $b(\hat{x}, \hat{s})$. Enunciamos este resultado no Lema 4.2, adaptado de [60, Teorema 3]. Novamente, reescrevemos a demonstração para fins de detalhamento do resultado.

Lema 4.2. Seja $x \in \mathbb{R}^{n}$ um ponto crítico de $P$. Seja ainda $p \in \mathbb{R}^{n}$ uma direção do tipo tunneling para $P$ a partir de $x$. Então, um minimizador global do problema

$$
\min _{\alpha \in \mathbb{R}} \phi_{x, s}(\alpha)
$$

é dado por

$$
\alpha= \begin{cases}\frac{3 b(x, s)+\sqrt{9 b(x, s)^{2}-32 a(x, s) c(x, s)}}{8 a(x, s)}, & \text { se } b(x, s) \geq 0, \\ \frac{3 b(x, s)-\sqrt{9 b(x, s)^{2}-32 a(x, s) c(x, s)}}{8 a(x, s)}, & \text { caso contrário. }\end{cases}
$$

Demonstração. Consideremos $x \in \mathbb{R}^{n}$ um ponto crítico de $P$ e $s \in \mathbb{R}^{n}$ uma direção de tipo tunneling de $P$ a partir de $x$. Como $\phi_{x, s}(\cdot)$ possui três pontos críticos (4.16), queremos mostrar qual dos três possui menor valor de função.

Para simplificar a notação nesta demonstração, consideraremos

$$
a \equiv a(x, s), b \equiv b(x, s), c \equiv c(x, s) \text { e } \Gamma \equiv \sqrt{9 b^{2}-32 a c} .
$$


Seguindo a notação, de (4.16) podemos reescrever

$$
\alpha_{1}=\frac{3 b+\Gamma}{8 a} \quad \text { e } \quad \alpha_{2}=\frac{3 b-\Gamma}{8 a} .
$$

Começaremos avaliando $\phi_{x, s}\left(\alpha_{1}\right)$. Temos que

$$
\begin{aligned}
& \alpha_{1}^{2}=\left(\frac{3 b+\Gamma}{8 a}\right)^{2}=\frac{9 b^{2}+6 b \Gamma+\Gamma^{2}}{64 a^{2}}, \\
\alpha_{1}^{3}= & \left(\frac{3 b+\Gamma}{8 a}\right)\left(\frac{9 b^{2}+6 b \Gamma+\Gamma^{2}}{64 a^{2}}\right) \\
= & \frac{27 b^{3}+18 b^{2} \Gamma+3 b \Gamma^{2}+9 b^{2} \Gamma+6 b \Gamma^{2}+\Gamma^{3}}{512 a^{3}} \\
= & \frac{27 b^{3}+27 b^{2} \Gamma+9 b \Gamma^{2}+\Gamma^{3}}{512 a^{3}}
\end{aligned}
$$

e

$$
\begin{aligned}
\alpha_{1}^{4} & =\left(\frac{3 b+\Gamma}{8 a}\right)\left(\frac{27 b^{3}+27 b^{2} \Gamma+9 b \Gamma^{2}+\Gamma^{3}}{512 a^{3}}\right) \\
& =\frac{81 b^{4}+81 b^{3} \Gamma+27 b^{2} \Gamma^{2}+3 b \Gamma^{3}+27 b^{3} \Gamma+27 b^{2} \Gamma^{2}+9 b \Gamma^{3}+\Gamma^{4}}{4096 a^{4}} \\
& =\frac{81 b^{4}+108 b^{3} \Gamma+54 b^{2} \Gamma^{2}+12 b \Gamma^{3}+\Gamma^{4}}{4096 a^{4}} .
\end{aligned}
$$

Por conseguinte, de (4.17), (4.18) e (4.19) segue que

$$
\begin{gathered}
a \alpha_{1}^{4}=\frac{81 b^{4}+108 b^{3} \Gamma+54 b^{2} \Gamma^{2}+12 b \Gamma^{3}+\Gamma^{4}}{4096 a^{3}}, \\
b \alpha_{1}^{3}=\frac{27 b^{4}+27 b^{3} \Gamma+9 b^{2} \Gamma^{2}+b \Gamma^{3}}{512 a^{3}}
\end{gathered}
$$

e

$$
\begin{aligned}
c \alpha_{1}^{2} & =\frac{9 b^{2} c+6 b c \Gamma+c \Gamma^{2}}{64 a^{2}} \\
& =\frac{9 b^{2} c+6 b c \Gamma+c\left(9 b^{2}-32 a c\right)}{64 a^{2}} \\
& =\frac{18 b^{2} c+6 b c \Gamma-32 a c^{2}}{64 a^{2}} \\
& =\frac{9 b^{2} c}{32 a^{2}}+\frac{3 b c \Gamma}{32 a^{2}}-\frac{c^{2}}{2 a} .
\end{aligned}
$$

De (4.20) e (4.21) temos que

$$
\begin{aligned}
a \alpha_{1}^{4}-b \alpha_{1}^{3} & =\frac{81 b^{4}+108 b^{3} \Gamma+54 b^{2} \Gamma^{2}+12 b \Gamma^{3}+\Gamma^{4}-216 b^{4}-216 b^{3} \Gamma-72 b^{2} \Gamma^{2}-8 b \Gamma^{3}}{4096 a^{3}} \\
& =\frac{-135 b^{4}-108 b^{3} \Gamma-18 b^{2} \Gamma^{2}+4 b \Gamma^{3}+\Gamma^{4}}{4096 a^{3}} .
\end{aligned}
$$


Todavia,

$$
18 b^{2} \Gamma^{2}=18 b^{2}\left(9 b^{2}-32 a c\right)=162 b^{4}-576 a b^{2} c
$$

e

$$
\Gamma^{4}=\left(9 b^{2}-32 a c\right)^{2}=81 b^{4}-576 a b^{2} c+1024 a^{2} c^{2} .
$$

Por (4.24) e (4.25) em (4.23) vem que

$$
\begin{aligned}
a \alpha_{1}^{4}-b \alpha_{1}^{3} & =\frac{-135 b^{4}-108 b^{3} \Gamma-162 b^{4}+576 a b^{2} c+4 b \Gamma^{3}+81 b^{4}-576 a b^{2} c+1024 a^{2} c^{2}}{4096 a^{3}} \\
& =\frac{-216 b^{4}-108 b^{3} \Gamma+4 b \Gamma^{3}+1024 a^{2} c^{2}}{4096 a^{3}} \\
& =-\frac{27 b^{4}}{512 a^{3}}-\frac{27 b^{3} \Gamma}{1024 a^{3}}+\frac{b \Gamma^{3}}{1024 a^{3}}+\frac{c^{2}}{4 a}
\end{aligned}
$$

Logo, por (4.22) e (4.26) temos que

$$
\begin{aligned}
a \alpha_{1}^{4}-b \alpha_{1}^{3}+c \alpha_{1}^{2} & =-\frac{27 b^{4}}{512 a^{3}}-\frac{27 b^{3} \Gamma}{1024 a^{3}}+\frac{b \Gamma^{3}}{1024 a^{3}}+\frac{c^{2}}{4 a}+\frac{9 b^{2} c}{32 a^{2}}+\frac{3 b c \Gamma}{32 a^{2}}-\frac{c^{2}}{2 a} \\
& =-\frac{c^{2}}{4 a}+\frac{9 b^{2} c}{32 a^{2}}-\frac{27 b^{4}}{512 a^{3}}+\frac{b \Gamma^{3}}{1024 a^{3}}+b \Gamma^{3}\left(\frac{3 c}{32 a^{2} \Gamma^{2}}-\frac{27 b^{2}}{1024 a^{3} \Gamma^{2}}\right) \\
& =-\frac{c^{2}}{4 a}+\frac{9 b^{2} c}{32 a^{2}}-\frac{27 b^{4}}{512 a^{3}}+\frac{b \Gamma^{3}}{1024 a^{3}}+b \Gamma^{3}\left(\frac{96 a c-27 b^{2}}{1024 a^{3} \Gamma^{2}}\right) \\
& =-\frac{c^{2}}{4 a}+\frac{9 b^{2} c}{32 a^{2}}-\frac{27 b^{4}}{512 a^{3}}+\frac{b \Gamma^{3}}{1024 a^{3}}-b \Gamma^{3}\left(\frac{3\left(9 b^{2}-32 a c\right)}{1024 a^{3} \Gamma^{2}}\right) \\
& =-\frac{c^{2}}{4 a}+\frac{9 b^{2} c}{32 a^{2}}-\frac{27 b^{4}}{512 a^{3}}+\frac{b \Gamma^{3}}{1024 a^{3}}-b \Gamma^{3}\left(\frac{3 \Gamma^{2}}{1024 a^{3} \Gamma^{2}}\right) \\
& =-\frac{c^{2}}{4 a}+\frac{9 b^{2} c}{32 a^{2}}-\frac{27 b^{4}}{512 a^{3}}-\frac{2 b \Gamma^{3}}{1024 a^{3}},
\end{aligned}
$$

donde segue que

$$
\phi_{x, s}\left(\alpha_{1}\right)=-\frac{c^{2}}{4 a}+\frac{9 b^{2} c}{32 a^{2}}-\frac{27 b^{4}}{512 a^{3}}-\frac{b \sqrt{\left(9 b^{2}-32 a c\right)^{3}}}{512 a^{3}}
$$

Analogamente, avaliando $\phi_{x, s}\left(\alpha_{2}\right)$ temos que

$$
\begin{aligned}
& \alpha_{2}^{2}=\left(\frac{3 b-\Gamma}{8 a}\right)^{2}=\frac{9 b^{2}-6 b \Gamma+\Gamma^{2}}{64 a^{2}}, \\
\alpha_{2}^{3}= & \left(\frac{3 b-\Gamma}{8 a}\right)\left(\frac{9 b^{2}-6 b \Gamma+\Gamma^{2}}{64 a^{2}}\right) \\
= & \frac{27 b^{3}-18 b^{2} \Gamma+3 b \Gamma^{2}-9 b^{2} \Gamma+6 b \Gamma^{2}-\Gamma^{3}}{512 a^{3}} \\
= & \frac{27 b^{3}-27 b^{2} \Gamma+9 b \Gamma^{2}-\Gamma^{3}}{512 a^{3}}
\end{aligned}
$$


e

$$
\begin{aligned}
\alpha_{2}^{4} & =\left(\frac{3 b-\Gamma}{8 a}\right)\left(\frac{27 b^{3}-27 b^{2} \Gamma+9 b \Gamma^{2}-\Gamma^{3}}{512 a^{3}}\right) \\
& =\frac{81 b^{4}-81 b^{3} \Gamma+27 b^{2} \Gamma^{2}-3 b \Gamma^{3}-27 b^{3} \Gamma+27 b^{2} \Gamma^{2}-9 b \Gamma^{3}+\Gamma^{4}}{4096 a^{4}} \\
& =\frac{81 b^{4}-108 b^{3} \Gamma+54 b^{2} \Gamma^{2}-12 b \Gamma^{3}+\Gamma^{4}}{4096 a^{4}} .
\end{aligned}
$$

Por conseguinte, de (4.28), (4.29) e (4.30) segue que

$$
\begin{gathered}
a \alpha_{2}^{4}=\frac{81 b^{4}-108 b^{3} \Gamma+54 b^{2} \Gamma^{2}-12 b \Gamma^{3}+\Gamma^{4}}{4096 a^{3}}, \\
b \alpha_{2}^{3}=\frac{27 b^{4}-27 b^{3} \Gamma+9 b^{2} \Gamma^{2}-b \Gamma^{3}}{512 a^{3}}
\end{gathered}
$$

e

$$
\begin{aligned}
c \alpha_{2}^{2} & =\frac{9 b^{2} c-6 b c \Gamma+c \Gamma^{2}}{64 a^{2}} \\
& =\frac{9 b^{2} c-6 b c \Gamma+c\left(9 b^{2}-32 a c\right)}{64 a^{2}} \\
& =\frac{18 b^{2} c-6 b c \Gamma-32 a c^{2}}{64 a^{2}} \\
& =\frac{9 b^{2} c}{32 a^{2}}-\frac{3 b c \Gamma}{32 a^{2}}-\frac{c^{2}}{2 a} .
\end{aligned}
$$

De (4.31) e (4.32) temos que

$$
\begin{aligned}
a \alpha_{2}^{4}-b \alpha_{2}^{3} & =\frac{81 b^{4}-108 b^{3} \Gamma+54 b^{2} \Gamma^{2}-12 b \Gamma^{3}+\Gamma^{4}-216 b^{4}+216 b^{3} \Gamma-72 b^{2} \Gamma^{2}+8 b \Gamma^{3}}{4096 a^{3}} \\
& =\frac{-135 b^{4}+108 b^{3} \Gamma-18 b^{2} \Gamma^{2}-4 b \Gamma^{3}+\Gamma^{4}}{4096 a^{3}} .
\end{aligned}
$$

Por (4.24) e (4.25) em (4.34) vem que

$$
\begin{aligned}
a \alpha_{2}^{4}-b \alpha_{2}^{3} & =\frac{-135 b^{4}+108 b^{3} \Gamma-162 b^{4}+576 a b^{2} c-4 b \Gamma^{3}+81 b^{4}-576 a b^{2} c+1024 a^{2} c^{2}}{4096 a^{3}} \\
& =\frac{-216 b^{4}+108 b^{3} \Gamma-4 b \Gamma^{3}+1024 a^{2} c^{2}}{4096 a^{3}} \\
& =-\frac{27 b^{4}}{512 a^{3}}+\frac{27 b^{3} \Gamma}{1024 a^{3}}-\frac{b \Gamma^{3}}{1024 a^{3}}+\frac{c^{2}}{4 a} .
\end{aligned}
$$


Logo, por (4.33) e (4.35) temos que

$$
\begin{aligned}
a \alpha_{2}^{4}-b \alpha_{2}^{3}+c \alpha_{2}^{2} & =-\frac{27 b^{4}}{512 a^{3}}+\frac{27 b^{3} \Gamma}{1024 a^{3}}-\frac{b \Gamma^{3}}{1024 a^{3}}+\frac{c^{2}}{4 a}+\frac{9 b^{2} c}{32 a^{2}}-\frac{3 b c \Gamma}{32 a^{2}}-\frac{c^{2}}{2 a} \\
& =-\frac{c^{2}}{4 a}+\frac{9 b^{2} c}{32 a^{2}}-\frac{27 b^{4}}{512 a^{3}}-\frac{b \Gamma^{3}}{1024 a^{3}}-b \Gamma^{3}\left(\frac{3 c}{32 a^{2} \Gamma^{2}}-\frac{27 b^{2}}{1024 a^{3} \Gamma^{2}}\right) \\
& =-\frac{c^{2}}{4 a}+\frac{9 b^{2} c}{32 a^{2}}-\frac{27 b^{4}}{512 a^{3}}-\frac{b \Gamma^{3}}{1024 a^{3}}-b \Gamma^{3}\left(\frac{96 a c-27 b^{2}}{1024 a^{3} \Gamma^{2}}\right) \\
& =-\frac{c^{2}}{4 a}+\frac{9 b^{2} c}{32 a^{2}}-\frac{27 b^{4}}{512 a^{3}}-\frac{b \Gamma^{3}}{1024 a^{3}}+b \Gamma^{3}\left(\frac{3\left(9 b^{2}-32 a c\right)}{1024 a^{3} \Gamma^{2}}\right) \\
& =-\frac{c^{2}}{4 a}+\frac{9 b^{2} c}{32 a^{2}}-\frac{27 b^{4}}{512 a^{3}}-\frac{b \Gamma^{3}}{1024 a^{3}}+b \Gamma^{3}\left(\frac{3 \Gamma^{2}}{1024 a^{3} \Gamma^{2}}\right) \\
& =-\frac{c^{2}}{4 a}+\frac{9 b^{2} c}{32 a^{2}}-\frac{27 b^{4}}{512 a^{3}}+\frac{2 b \Gamma^{3}}{1024 a^{3}},
\end{aligned}
$$

donde segue que

$$
\phi_{x, s}\left(\alpha_{2}\right)=-\frac{c^{2}}{4 a}+\frac{9 b^{2} c}{32 a^{2}}-\frac{27 b^{4}}{512 a^{3}}+\frac{b \sqrt{\left(9 b^{2}-32 a c\right)^{3}}}{512 a^{3}}
$$

Deste modo, se $b \geq 0$, então de (4.27) temos que

$$
\phi_{x, s}\left(\alpha_{1}\right)=-\frac{c^{2}}{4 a}+\frac{9 b^{2} c}{32 a^{2}}-\frac{27 b^{4}}{512 a^{3}}-\frac{\sqrt{b^{2}\left(9 b^{2}-32 a c\right)^{3}}}{512 a^{3}}
$$

e de (4.36) que

$$
\phi_{x, s}\left(\alpha_{2}\right)=-\frac{c^{2}}{4 a}+\frac{9 b^{2} c}{32 a^{2}}-\frac{27 b^{4}}{512 a^{3}}+\frac{\sqrt{b^{2}\left(9 b^{2}-32 a c\right)^{3}}}{512 a^{3}} .
$$

Neste caso, $\phi_{x, s}\left(\alpha_{1}\right) \leq \phi_{x, s}\left(\alpha_{2}\right)$. Para constatarmos que $\alpha_{1}$ é minimizador global de $\phi_{x, s}(\cdot)$, resta-nos mostrar que $\phi\left(\alpha_{1}\right)<0=\phi\left(\alpha_{0}\right)$. Como $a>0$, se $c<0$ então $\phi_{x, s}\left(\alpha_{1}\right)<0$. Suponhamos então que $c>0$. Como $\Delta(x, s)>0, b^{2}-4 a c>0$, ou seja, $b^{2}>4 a c$. Logo,

$$
\begin{aligned}
\phi_{x, s}\left(\alpha_{1}\right) & =-\frac{c^{2}}{4 a}+\frac{9 b^{2} c}{32 a^{2}}-\frac{27 b^{4}}{512 a^{3}}-\frac{\sqrt{b^{2}\left(9 b^{2}-32 a c\right)^{3}}}{512 a^{3}} \\
& <-\frac{c^{2}}{4 a}+\frac{9 b^{2} c}{32 a^{2}}-\frac{27 b^{4}}{512 a^{3}}-\frac{\sqrt{b^{2}\left(9 b^{2}-8 b^{2}\right)^{3}}}{512 a^{3}} \\
& =-\frac{c^{2}}{4 a}+\frac{9 b^{2} c}{32 a^{2}}-\frac{27 b^{4}}{512 a^{3}}-\frac{b^{4}}{512 a^{3}} \\
& =-\frac{c^{2}}{4 a}+\frac{9 b^{2} c}{32 a^{2}}-\frac{7 b^{4}}{128 a^{3}} \\
& =\frac{-32 a^{2} c^{2}+36 a b^{2} c-7 b^{4}}{128 a^{3}} \\
& =-\frac{\left(7 b^{2}-8 a c\right)\left(b^{2}-4 a c\right)}{128 a^{3}} .
\end{aligned}
$$

Como $b^{2}>0, a>0, c>0$ e $b^{2}-4 a c>0$, então $7 b^{2}-4 a c>0$ e, por (4.37), temos que $\phi_{x, s}\left(\alpha_{1}\right)<0$. Portanto $\alpha_{1}$ é minimizador global de $\phi_{x, s}(\cdot)$. 
Analogamente, se $b<0$, então de (4.27) temos que

$$
\phi_{x, s}\left(\alpha_{1}\right)=-\frac{c^{2}}{4 a}+\frac{9 b^{2} c}{32 a^{2}}-\frac{27 b^{4}}{512 a^{3}}+\frac{\sqrt{b^{2}\left(9 b^{2}-32 a c\right)^{3}}}{512 a^{3}}
$$

e de (4.36) que

$$
\phi_{x, s}\left(\alpha_{2}\right)=-\frac{c^{2}}{4 a}+\frac{9 b^{2} c}{32 a^{2}}-\frac{27 b^{4}}{512 a^{3}}-\frac{\sqrt{b^{2}\left(9 b^{2}-32 a c\right)^{3}}}{512 a^{3}} .
$$

Neste caso, $\phi_{x, s}\left(\alpha_{2}\right) \leq \phi_{x, s}\left(\alpha_{1}\right)$ e, por $(4.37), \phi_{x, s}\left(\alpha_{2}\right)<0$. Por conseguinte, $\alpha_{2}$ é minimizador global de $\phi_{x, s}(\cdot)$.

Temos até aqui todos os ingredientes para descrever o método no Algoritmo 4.1.

\section{Algoritmo 4.1: Minimização de polinômios quárticos normais}

Passo 0. Inicialização. Dados um ponto inicial $x^{0} \in \mathbb{R}^{n}, \rho>2, \epsilon_{0} \geq 0, \epsilon_{1}, \epsilon_{2}>0, \ell \geq 0$. Consideremos $\phi_{x, s}(\cdot)$ definida em $(4.12)$ e $P(\cdot)$ um polinômio quártico normal. Inicialize $k \leftarrow 0$.

\section{Passo 1. Ponto não crítico. Se}

$$
\left\|\nabla P\left(x^{k}\right)\right\| \leq \epsilon_{0}
$$

vá para o Passo 2. Senão, calcule o passo de Newton $s^{N}$ dado pela resolução do sistema linear

$$
\nabla^{2} P\left(x^{k}\right) s^{N}=-\nabla P\left(x^{k}\right) .
$$

Se $s^{N}$ não existir ou se

$$
\nabla P\left(x^{k}\right)^{T} s^{N} \geq-\epsilon_{1}\left\|p^{N}\right\|^{\rho},
$$

tome $s^{k}=-\nabla P\left(x^{k}\right)$, senão, $s^{k}=s^{N}$. Depois, encontre um minimizador global $\alpha_{k}$ do problema

$$
\min _{\alpha \in \mathbb{R}} \phi_{x^{k}, s^{k}}(\alpha)
$$

calculando os zeros de $\phi_{x^{k}, s^{k}}^{\prime}(\cdot)$. Calcule $x^{k+1}=x^{k}+\alpha_{k} s^{k}$, faça $k \leftarrow k+1$ e repita o Passo 1 .

Passo 2. Ponto crítico. Seja $\hat{\lambda}$ o menor autovalor de $\nabla^{2} P\left(x^{k}\right)$.

2.1. Maximizador local ou ponto de sela. Se $\hat{\lambda} \leq 0$ então calcule um autovetor $s^{k}$ associado a $\hat{\lambda}$. Se

$$
(\hat{\lambda}<0) \text { ou }\left(\hat{\lambda}=0 \text { e } b\left(x^{k}, s^{k}\right) \neq 0\right)
$$

calcule

$$
\alpha_{k}= \begin{cases}\frac{3 b\left(x^{k}, s^{k}\right)+\sqrt{9 b\left(x^{k}, s^{k}\right)^{2}-32 a\left(x^{k}, s^{k}\right) c\left(x^{k}, s^{k}\right)}}{8 a\left(x^{k}, s^{k}\right)}, & \text { se } b\left(x^{k}, s^{k}\right) \geq 0, \\ \frac{3 b\left(x^{k}, s^{k}\right)-\sqrt{9 b\left(x^{k}, s^{k}\right)^{2}-32 a\left(x^{k}, s^{k}\right) c\left(x^{k}, s^{k}\right)}}{8 a\left(x^{k}, s^{k}\right)}, & \text { caso contrário, }\end{cases}
$$

defina $x^{k+1}=x^{k}+\alpha_{k} s^{k}$, faça $k \leftarrow k+1$ e vá para o Passo 1 .

2.2. Minimizador local. 
2.2.1. Seja $\mathcal{V}=\left\{v^{k, 1}, v^{k, 2}, \ldots, v^{k, \ell}\right\}$ um conjunto de vetores em $\mathbb{R}^{n}$. Se $\Delta\left(x^{k}, v\right)>0$ para algum $v \in \mathcal{V}$, então tome $s^{k}=v$, calcule $\alpha_{k}$ usando (4.38), defina $x^{k+1}=x^{k}+\alpha_{k} s^{k}$, faça $k \leftarrow k+1$ e vá para o Passo 1 . Senão, pare e retorne $x^{k}$.

2.2.2. Calcule um ponto viável $\left(\bar{x}, \bar{x}_{n+1}\right)$ para o sistema não linear

$$
\left\{\begin{array}{cl}
\nabla P(x) & =0 \\
P(x)-x_{n+1} & =0 \\
x_{n+1}-P(\hat{x})+\epsilon & \leq 0
\end{array}\right.
$$

Se $\bar{x}$ for computado com sucesso, tome $x^{k+1}=\bar{x}$, faça $k \leftarrow k+1$ e vá para o Passo 1 . Senão, pare e retorne $x^{k}$.

\subsubsection{Gloptipoly: um método para minimização global de polinômios}

Gloptipoly é um método para minimização global de polinômios, implementado em Matlab ${ }^{\circledR}$ proposto por Henrion e Lasserre [46] baseado na teoria de polinômios e problema de momentos $[1,4,38,62]$ desenvolvido por Lasserre [47-50]. Foi concebido para resolução do problema

$$
\begin{aligned}
\text { Minimizar } & p(x) \\
\text { s. a } & P_{k}(x) \geq 0, k=\{1, \ldots, m\},
\end{aligned}
$$

onde $p(x), P_{k}(x)$, para $k=1, \ldots, m$, são polinômios reais de $\mathbb{R}^{n}$ em $\mathbb{R}$.

Na presente seção, daremos uma descrição acerca do Gloptipoly contextualizada à nossa aplicação, que é a resolução do problema (4.1). Por se tratar de um problema irrestrito, descreveremos Gloptipoly para o caso em que $m=0$, ou seja, para o problema

$$
\underset{x \in \mathbb{R}^{n}}{\operatorname{Minimizar}} p(x) \text {. }
$$

Seja

$$
\mathbb{B}=\left\{1, x_{1}, x_{2}, \ldots, x_{n}, x_{1}^{2}, x_{1} x_{2}, \ldots, x_{1} x_{n}, x_{2}^{2}, x_{2} x_{3}, \ldots, x_{2} x_{n}, \ldots, x_{n}^{2}, \ldots, x_{1}^{r}, \ldots, x_{n}^{r}\right\}
$$

uma base para o espaço dos polinômios de grau no máximo $r$. Como $\mathbb{B}$ contém todos os monômios possíveis de um polinômio de grau no máximo $r$, então sua dimensão é dada por

$$
s(r)=\left(\begin{array}{c}
n+r \\
r
\end{array}\right)=\frac{(n+r) !}{n ! r !} .
$$

Seja $\alpha$ um vetor de dimensão $s(r)$ tal que

$$
\alpha \in \mathbb{N}^{s(r)}, \alpha_{i}=i_{1} i_{2} \ldots i_{n}, \text { para } i=1, \ldots, s(r),
$$

de tal forma que o $i$-ésimo monômio da base $\mathbb{B}$ seja $x_{1}^{i_{1}} x_{2}^{i_{2}} \ldots x_{n}^{i_{n}}$. Para simplificar nossa notação, consideraremos $x^{\alpha_{i}}=x_{1}^{i_{1}} x_{2}^{i_{2}} \ldots x_{n}^{i_{n}}$.

Para fixar ideias, tomemos como exemplo o caso em que $n=2$ e $r=2$. Então, podemos definir uma base dada por

e, neste caso, teríamos

$$
\mathbb{B}=\left\{1, x_{1}, x_{2}, x_{1}^{2}, x_{1} x_{2}, x_{2}^{2}\right\},
$$

$$
\alpha=(00,10,01,20,11,02)^{T} .
$$

Consideremos a seguinte definição. 
Definição 4.1. Matriz de momento Seja $r>0$ e $\mathbb{B}$ uma base para o espaço dos polinômios de grau no máximo $r$ com dimensão $s(r)$. Seja $y \in \mathbb{R}^{s(r)}, y=\left(y_{\alpha_{1}}, y_{\alpha_{2}}, \ldots, y_{\alpha_{s(r)}}\right)^{T}$ com $\alpha$ como definido em (4.40). Chamaremos de matriz de momento de ordem $r$ a matriz $M_{r} \in \mathbb{R}^{s(r) \times s(r)}$ dada por

$$
M_{r}(y)=\left[\begin{array}{cccc}
y_{\alpha_{1}} & y_{\alpha_{2}} & \ldots & y_{\alpha_{s(r)}} \\
y_{\alpha_{2}} & y_{\alpha_{2}+\alpha_{2}} & \ldots & y_{\alpha_{2}+\alpha_{s(r)}} \\
\vdots & \vdots & \vdots & \vdots \\
y_{\alpha_{s(r)}} & y_{\alpha_{s(r)}+\alpha_{2}} & \ldots & y_{\alpha_{s(r)}+\alpha_{s(r)}}
\end{array}\right]
$$

Novamente, para fixar ideias, consideremos o caso em que $n=2$ e $r=2$. Teríamos que

$$
M_{2}(y)=\left[\begin{array}{cccccc}
1 & y_{10} & y_{01} & y_{20} & y_{11} & y_{02} \\
y_{10} & y_{20} & y_{11} & y_{30} & y_{21} & y_{12} \\
y_{01} & y_{11} & y_{02} & y_{21} & y_{12} & y_{03} \\
y_{20} & y_{30} & y_{21} & y_{40} & y_{31} & y_{22} \\
y_{11} & y_{21} & y_{12} & y_{31} & y_{22} & y_{13} \\
y_{02} & y_{12} & y_{03} & y_{22} & y_{13} & y_{04}
\end{array}\right] \text {. }
$$

Consideraremos que

$$
p(x)=\sum_{i=1}^{s(r)} p_{\alpha_{i}} x^{\alpha_{i}} .
$$

Denotaremos simplesmente por $p=\left(p_{\alpha_{1}}, p_{\alpha_{2}}, \ldots, p_{\alpha_{s(r)}}\right)^{T} \in \mathbb{R}^{s(r)}$ o vetor cujas componentes são coeficientes de $p(x)$ na base (4.39).

Seja o problema de programação semidefinida

$$
\begin{aligned}
\underset{y}{\operatorname{Minimizar}} & \sum_{i=1}^{s(r)} p_{\alpha_{i}} y_{\alpha_{i}} \\
\text { s. a } & M_{r}(y) \succeq 0,
\end{aligned}
$$

onde $M_{r}(y)$ é uma matriz de momento de ordem $r$ e $M_{r}(y) \succeq 0$ denota que $M_{r}$ é semidefinida positiva. Se considerarmos $M_{r}=B_{0}+\sum_{i=2}^{s(r)} y_{\alpha_{i}} B_{\alpha_{i}}$, onde $B_{0}$ é a matriz nula exceto por conter 1 na entrada $(1,1)$ e as matrizes $B_{\alpha_{i}}$, para $i=2, \ldots, s(r)$ adequadamente definidas, então podemos reescrever $\mathbb{Q}$ como

$$
\begin{aligned}
\underset{y}{\operatorname{Minimizar}} & \sum_{i=1}^{s(r)} p_{\alpha_{i}} y_{\alpha_{i}} \\
\text { s. a } & \sum_{i=2}^{s(r)} y_{\alpha_{i}} B_{\alpha_{i}} \succeq-B_{0} .
\end{aligned}
$$

O dual de $\mathbb{Q}$ é dado por

$$
\begin{aligned}
& \max _{X}\left\langle X,-B_{0}\right\rangle\left(=-X_{1,1}\right) \\
& \text { s. a }\left\langle X, B_{\alpha_{i}}\right\rangle=p_{\alpha_{i}} \text {, para } i=2, \ldots, s(r) \text {, } \\
& X \succeq 0 \text {, }
\end{aligned}
$$

onde $X$ é uma matriz real simétrica e $\langle A, B\rangle$ denota o produto interno de Frobenius dado por $\operatorname{Tr}(A B)$ para matrizes simétricas $A$ e $B$, sendo $\operatorname{Tr}(\cdot)$ o traço de uma matriz.

Vale o seguinte resultado. 
Teorema 4.3. Seja $p: \mathbb{R}^{n} \rightarrow \mathbb{R}$ um polinômio de grau $r$ como definido em (4.41). Suponha que $p(x)$ possua minimo global $p^{*}$.

i. Se o polinômio não negativo $p(x)-p^{*}$ puder ser escrito como uma soma de polinômios ao quadrado, isto é, se

$$
p(x)-p^{*}=\sum_{i} q_{i}(x)^{2},
$$

para polinômios $q_{i}(x): \mathbb{R}^{n} \rightarrow \mathbb{R}$, então o problema $\mathbb{P}$ é equivalente ao problema de programação semidefinida $\mathbb{Q}$. Precisamente, os mínimos globais de $\mathbb{P}$ e $\mathbb{Q}$ coincidem e se $x^{*}$ é minimizador global de $\mathbb{P}$, então

$$
y^{*}=\left(x_{1}^{*}, x_{2}^{*}, \ldots, x_{n}^{*},\left(x_{1}^{*}\right)^{2}, x_{1}^{*} x_{2}^{*}, \ldots,\left(x_{1}^{*}\right)^{r}, \ldots,\left(x_{n}^{*}\right)^{r}\right)^{T}
$$

é minimizador global de $\mathbb{Q}$.

ii. Se $\mathbb{Q}^{*}$ possuir uma solução viável, então os mínimos globais de $\mathbb{P} e \mathbb{Q}$ coincidirão somente se $p(x)-p^{*}$ puder ser escrito como uma soma de polinômios ao quadrado.

Demonstração. Ver demonstração do Teorema 3.2 [47, p. 801].

O Teorema 4.3 exige uma condição forte: que o polinômio $p(x)-p^{*}$ possa ser escrito como uma soma de polinômios ao quadrado. Esta condição pode ser relaxada caso saibamos, a priori, que o minimizador global de $p(x)$ é limitado, isto é, que $\left\|x^{*}\right\| \leq a$ para algum $a>0$.

Para tanto consideremos a seguinte notação: seja

$$
\theta(x)=a^{2}-\|x\|^{2}
$$

e $q: \mathbb{R}^{n} \rightarrow \mathbb{R}$ um polinômio de grau $w$ com vetor de coeficientes $q \in \mathbb{R}^{s(w)}$ na base de polinômios de grau no máximo $w$ indexado pelo vetor $\alpha$ como definido em (4.40). Seja ainda $M_{r}(y)$ uma matriz de momento de ordem $r$. Consideremos $\beta_{i j}$ o subíndice de $y$ na posição $(i, j)$ de $M_{r}(y)$. Consideraremos $M_{r}(q y)$ a matriz dada por

$$
\left[M_{r}(q y)\right]_{i, j}=\sum_{\ell=1}^{s(w)} q_{\alpha_{\ell}} y_{\beta_{i j}+\alpha_{\ell}} .
$$

Para fixar ideias, consideremos

$$
M_{1}(y)=\left[\begin{array}{ccc}
1 & y_{10} & y_{01} \\
y_{10} & y_{20} & y_{11} \\
y_{01} & y_{11} & y_{02}
\end{array}\right] \text { e } q(x)=a-x_{1}^{2}-x_{2}^{2} .
$$

Desta forma, como $q$ é um polinômio de grau 2, consideraremos a base

$$
\mathbb{B}=\left\{1, x_{1}, x_{2}, x_{1}^{2}, x_{1} x_{2}, x_{2}^{2}\right\},
$$

o vetor

$$
\alpha=(00,10,01,20,11,02)^{T}
$$

e

$$
q=\left(\begin{array}{llllll}
q_{00} & q_{10} & q_{01} & q_{20} & q_{11} & q_{02}
\end{array}\right)^{T}=(a, 0,0,-1,0,-1)^{T} .
$$


Deste modo,

$$
M_{1}(q y)=\left[\begin{array}{ccc}
a-y_{20}-y_{02} & a y_{10}-y_{30}-y_{12} & a y_{01}-y_{21}-y_{03} \\
a y_{10}-y_{30}-y_{12} & a y_{20}-y_{40}-y_{22} & a y_{11}-y_{31}-y_{13} \\
a y_{01}-y_{21}-y_{03} & a y_{11}-y_{31}-y_{13} & a y_{02}-y_{22}-y_{04}
\end{array}\right] .
$$

Para $N>r / 2$, consideremos o problema de programação semidefinida

$$
\begin{aligned}
\underset{y}{\operatorname{Minimizar}} & \sum_{i=1}^{s(N)} p_{\alpha_{i}} y_{\alpha_{i}} \\
\text { s. a } & M_{N}(y) \succeq 0, \\
& M_{N-1}(\theta y) \succeq 0 .
\end{aligned}
$$

Se considerarmos $M_{N-1}(q y)=\sum_{i=1}^{s(N-1)} y_{\alpha_{i}} C_{\alpha_{i}}$ com matrizes $C_{\alpha}$ adequadamente definidas, o dual de $\mathbb{Q}_{a}^{N}$ é dado por

$$
\begin{aligned}
\max _{X, Z}-X_{1,1}-a^{2} Z_{1,1} & \\
\text { s. a }\left\langle X, B_{\alpha_{i+1}}\right\rangle+\left\langle Z, C_{\alpha_{i}}\right\rangle & =p_{\alpha_{i}}, \text { para } i=1, \ldots, N-1, \\
X & \succeq 0, \\
Z & \succeq 0 .
\end{aligned}
$$

Então, vale o seguinte resultado.

Teorema 4.4. Seja $p: \mathbb{R}^{n} \rightarrow \mathbb{R}$ um polinômio de grau no máximo $r$ como definido em (4.41) com minimo global $p^{*}=p\left(x^{*}\right)$ tal que $\left\|x^{*}\right\| \leq$ a para algum $a>0$. Então,

a. $\lim _{N \rightarrow \infty} \inf \mathbb{Q}_{a}^{N}=p^{*}$.

b. $\min \mathbb{Q}_{a}^{N}=p^{*}$ se e somente se

$$
p(x)-p^{*}=\sum_{i=1}^{r_{1}} q_{i}(x)^{2}+\theta(x) \sum_{j=1}^{r_{2}} t_{j}(x)^{2}
$$

para polinômios $q_{i}(x), i=1, \ldots, r_{1}$, de grau no máximo $N$, e $t_{j}(x), j=1, \ldots, r_{2}$, de grau no máximo $N-1$. Neste caso, o vetor

$$
y^{*}=\left(x_{1}^{*}, \ldots, x_{n}^{*},\left(x_{1}^{*}\right)^{2}, x_{1}^{*} x_{2}^{*}, \ldots,\left(x_{1}^{*}\right)^{2 N}, \ldots,\left(x_{n}^{*}\right)^{2 N}\right)^{T}
$$

é um minimizador de $\mathbb{Q}_{a}^{N}$. Além disso, $\max \left(\mathbb{Q}_{a}^{N}\right)^{*}=\min \mathbb{Q}_{a}^{N}$ e para todo maximizador $\left(X^{*}, Z^{*}\right)$ de $\left(\mathbb{Q}_{a}^{N}\right)^{*}$,

$$
p(x)-p^{*}=\sum_{i=1}^{r_{1}} \lambda_{i} q_{i}(x)^{2}+\theta(x) \sum_{j=1}^{r_{2}} \gamma_{j} t_{j}(x)^{2},
$$

onde o vetor dos coeficientes dos polinômios $q_{i}(x), i=1, \ldots, r_{1}$ e $t_{j}(x), j=1, \ldots, r_{2}$ são os autovetores de $X^{*}$ e $Z^{*}$ com respectivos autovalores $\lambda_{i}, i=1, \ldots, r_{1}$ e $\gamma_{j}, j=1, \ldots, r_{2}$.

Demonstração. Ver Teorema 3.4 [47, p. 805]. 
Em [4, p. 119], o autor estabelece que um polinômio $p(x)$ pode ser escrito na forma

$$
p(x)=\sum_{i=1}^{r_{1}} q_{i}(x)^{2}+\theta(x) \sum_{j=1}^{r_{2}} t_{j}(x)^{2},
$$

para polinômios $q_{i}, t_{j}, i=1, \ldots, r_{1}, j=1, \ldots, r_{2}$, somente se $p(x)$ for estritamente positivo no conjunto $K=\left\{x \in \mathbb{R}^{n} \mid \theta(x) \geq 0\right\}$. Não obstante, o problema fica em aberto para os casos em que $p(x)$ seja apenas não negativo em $K$. Vale notar que $p(x)-p^{*}$ é não negativo em $K$. Lassere [47] argumenta que, na prática, Gloptipoly se comporta muito bem, principalmente se os problemas de programação semidefinida forem resolvidos utilizando um método de pontos interiores.

Na prática, ao executar o método Gloptipoly, três coisas podem acontecer [46]:

1. Gloptipoly pode detectar que o $\mathbb{Q}_{a}^{N}$ é inviável ou não pode ser resolvido.

Este caso pode ocorrer por alguns motivos. Em primeiro lugar, por problemas numéricos, pode ser que a precisão desejada não possa ser alcançada pelo método para resolver problemas de programação semidefinida. Em segundo lugar, pode ser que $\left(\mathbb{Q}_{a}^{N}\right)^{*}$ seja inviável, desta forma $\mathbb{Q}_{a}^{N}$ também será, o que significa que o problema original também é inviável (para o caso de minimização com restrições). Em terceiro e último lugar, pode ser que $\mathbb{Q}_{a}^{N}$ seja inviável, o que implica que $\left(\mathbb{Q}_{a}^{N}\right)^{*}$ é ilimitado. Uma solução para isso é adicionar restrições de compacidade às variáveis do problema original. Uma forma de fazer isso é adicionar uma restrição de bola do tipo $\|x\|^{2} \leq a^{2}$ ao problema original. Se $a$ for suficientemente grande de tal forma a fazer com que a bola contenha o minimizador global do problema irrestrito, o problema com uma tal restrição é equivalente ao problema original irrestrito.

2. O problema $\mathbb{Q}_{a}^{N}$ foi resolvido, mas não foi possivel atestar otimalidade global para $N$ tomado. De acordo com [46], a otimalidade global, em nosso caso, é atestada quando (i) as variáveis y do problema $\mathbb{Q}_{a}^{N}$ satisfazem todas as restrições do problema original e quando (ii) $\operatorname{posto}\left(M_{N}\right)=$ posto $\left(M_{N-1}\right)$, para algum $N>r / 2$. Neste caso, pode ser que Gloptipoly apenas obteve um limitante inferior para o mínimo global do problema original. O que se deve fazer, neste caso, é aumentar $N$.

3. O problema $\mathbb{Q}_{a}^{N}$ foi resolvido, a otimalidade global atestada e o minimizador global extraído com sucesso.

Neste caso, tudo ocorreu bem e o método conseguiu extrair o minimizador global.

Experimentos numéricos com Gloptipoly revelaram deficiências numéricas do método. Vamos ilustrá-la com um exemplo. Consideremos a função de uma variável

$$
m(d)=0.96617+6.8644 d+13.7819 d^{2}+14.7864 d^{3}+0.25 d^{4} .
$$

Rodando Gloptipoly com valores padrões para suas constantes, o algoritmo concluía que o problema de programação semidefinida era ilimitado inferiormente:

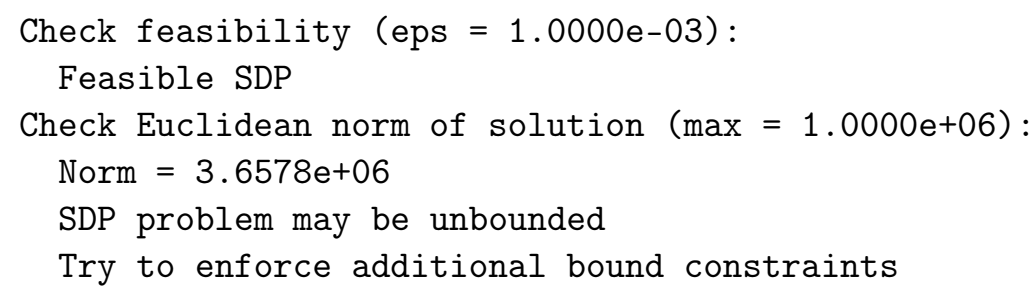


Moment SDP could not be solved

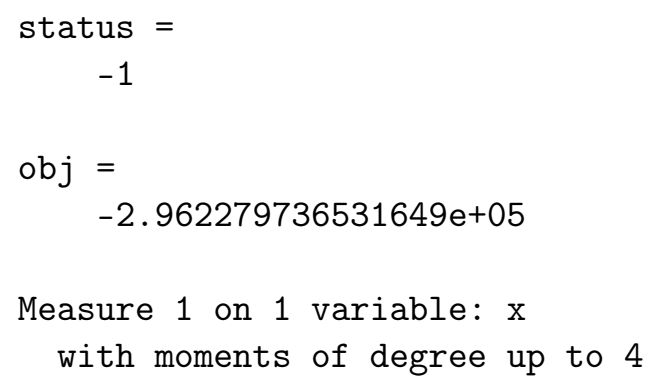

Isto acontecia porque a norma $3.6578 \times 10^{6}$ da solução era maior que o limiar padrão $10^{6}$ de Gloptipoly. Definindo este limiar como infinito, Gloptipoly consegue obter a solução global, mas podemos reparar que a norma da solução é razoavelmente grande:

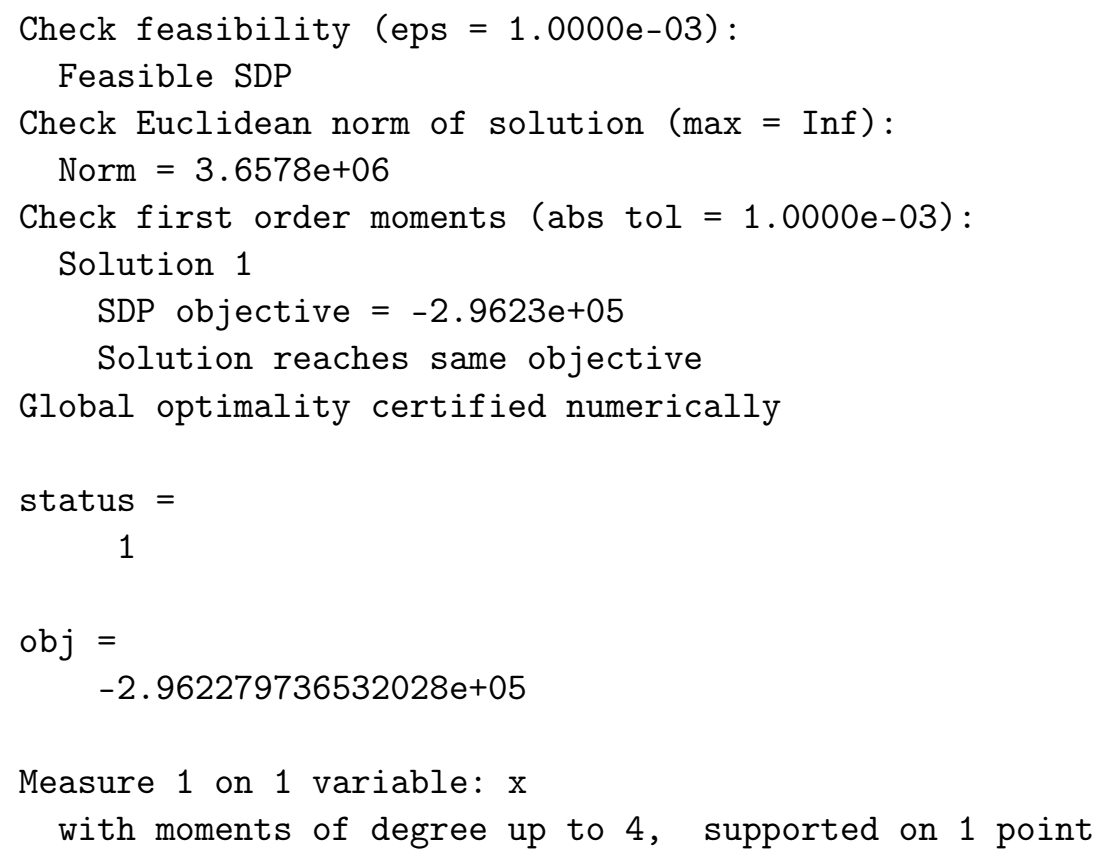

Neste caso, a solução retornada por Gloptipoly é $d^{*}=-43.7296$. Como o grau de $m(d)$ é quatro e o polinômio é unidimensional, consideramos a base

$$
B=\left\{1, d, d^{2}, d^{3}, d^{4}\right\}
$$

De fato,

$$
\left\|y^{*}\right\|_{2}=\left\|\left(y_{0}^{*} y_{1}^{*} y_{2}^{*} y_{3}^{*} y_{4}^{*}\right)^{T}\right\|_{2}=\left\|\left(1 d^{*}\left(d^{*}\right)^{2}\left(d^{*}\right)^{3}\left(d^{*}\right)^{4}\right)^{T}\right\|_{2} \approx 3.657765257802357 \times 10^{6} .
$$

O que podemos concluir deste exemplo é que, se $\mathbb{P}$ possuir minimizador com norma grande, então o minimizador de $\mathbb{Q}_{a}^{N}$ possuirá norma bastante maior. Isso acontece por causa da forma da base considerada para o espaço dos polinômios. Os experimentos com $\mathrm{AR}_{3}$ confirmarão esta característica de Gloptipoly. 


\subsection{Experimentos com Gloptipoly e GMNQP}

Para os experimentos numéricos, rodamos Gloptipoly versão $3.8^{2}$ usando Matlab ${ }^{\circledR}$ R2015a em um iMac OS X Yosemite com Intel ${ }^{\circledR}$ Core $^{\text {TM }}$ i5 3,4 GHz. Os problemas teste foram os mesmos tomados por Qi, Wan e Yang [60, p. 298].

Os resultados dos experimentos são apresentados na Tabela 4.1. Nela, reportamos os números obtidos com Gloptipoly e colocamos os mesmos obtidos por Qi, Wan e Yang [60, p. 296-298].

\begin{tabular}{|r|r|r||r|r|r|}
\hline prob & Gloptipoly & GMNQP & prob & Gloptipoly & GMNQP \\
\hline \hline Q21 & -10.788971 & -10.788971 & Q36 & -17687.106628 & -17687.1075 \\
Q22 & -17.362005 & -17.362004 & Q37 & -70.116764 & -70.116764 \\
Q23 & -31.140192 & -31.140191 & Q38 & -17733.905843 & -102.236381 \\
Q24 & -92.283429 & -92.283429 & Q39 & -147.457860 & -147.45786 \\
Q25 & -267.742055 & 1.551273 & Q310 & -31876.984955 & -31876.9903 \\
Q26 & -23248.809994 & 1288925.946058 & Q41 & -1395.233902 & -1395.23409 \\
Q27 & -103.344964 & -103.344964 & Q42 & -222.071570 & -222.071582 \\
Q28 & 56.948473 & 56.948472 & Q43 & -117.921857 & -117.921805 \\
Q29 & -83.955000 & -83.955001 & Q44 & -122.447305 & -122.44731 \\
Q210 & -15.385642 & -15.385642 & Q45 & -201.373673 & -201.373676 \\
Q31 & -112.374933 & -112.374935 & Q61 & -29.197812 & 28.9428173 \\
Q32 & -22.110069 & -22.110068 & Q62 & -23.005648 & -23.0056478 \\
Q33 & -228.406947 & -228.406952 & Q63 & -31.780369 & -16.2724185 \\
Q34 & -748.700563 & -748.700592 & Q64 & -70.878178 & -70.8781817 \\
Q35 & -301.716293 & -280.103848 & & & \\
\hline
\end{tabular}

Tabela 4.1: Resultado do teste comparativo entre GMNQP e Gloptipoly. Os resultados de GMNQP foram reproduzidos de [60], todavia com as casas decimais truncadas para, no máximo, seis. Em destaque, os problemas em que Gloptipoly se sobressai. Em nenhum GMNQP obteve melhores resultados.

Como podemos observar, em seis casos Gloptipoly obtém soluções cuja função objetivo vale menos que na solução encontrada por GMNQP. Por conseguinte, este experimento mostra que GMNQP não é um método prático para minimização global.

\subsection{Experimentos numéricos com $\mathrm{AR}_{3}$}

Para a realização de nossos experimentos, tomamos polinômios de grau seis da forma

$$
P(x)=\sum_{i=1}^{n} x_{i}^{6}+p_{5}(x),
$$

onde $p_{5}(x)$ é um polinômio qualquer de grau no máximo 5. Escolhemos problemas desta forma por dois motivos. Por um lado, polinômios constituem a classe mais simples de funções em nosso contexto de experimentos, isto é, tanto para controle de bom escalamento da função objetivo e de suas derivadas (para isso, basta controlar a magnitude dos coeficientes do polinômio) quanto pela simplicidade em computar derivadas de qualquer ordem. Por outro lado, como o modelo (4.1) trata-se de um polinômio de grau no máximo quatro, faz sentido tomar polinômios de grau seis da forma de (4.43) por não se assemelharem ao modelo a ser minimizado em cada subproblema de $\mathrm{AR}_{3}$ e por serem limitados inferiormente, já que $\lim _{x \rightarrow \infty} P(x)=\infty$ e $\lim _{x \rightarrow-\infty} P(x)=\infty$.

\footnotetext{
${ }^{2}$ A última versão de Gloptipoly até a presente data, pode ser obtida no endereço http://homepages.laas.fr/ henrion/software/gloptipoly/
} 
Para os valores das constantes do Algoritmo 2.2, seguimos as sugestões de [21] na implementação do método de regularização cúbica e consideramos $\sigma_{0}=1, \eta_{1}=0.1$ e $\eta_{2}=0.9$. Ademais, tomamos $\epsilon=10^{-8}, \gamma_{1}=0.5, \gamma_{2}=1.0, \gamma_{3}=2.0, \sigma_{\min }=10^{-8}$ e $\theta=10^{16}$.

Separamos nosso conjunto de testes em polinômios densos e esparsos. Neste contexto, consideramos a seguinte definição.

Definição 4.2. Polinômio esparso. Dado um polinômio de grau no máximo $\ell$ com n variáveis,

$$
T_{n, \ell}=\left(\begin{array}{c}
n+\ell \\
\ell
\end{array}\right)=\frac{(n+\ell) !}{\ell ! n !}
$$

é a quantidade máxima de monômios deste polinômio. Consideremos $P_{\ell}(x)$ um polinômio de grau no máximo $\ell$ com $m$ monômios. Então, consideraremos que $P_{\ell}$ é esparso se

$$
\frac{m}{T_{n, \ell}} \ll 1 .
$$

Os polinômios esparsos que tomamos para nosso experimento são da forma definida por $P$ em (4.43) tal que $p_{5}$ tem $\min \left\{T_{n, 5}, 10\right\}$ monômios. Os polinômios foram gerados aleatoriamente, com seus coeficientes variando entre -1 e 1 para não termos problemas mal escalados (em especial, conforme $n$ cresce, o mal escalamento do problema também cresce).

Experimentamos as implementações $\mathrm{AR}_{3}^{G}$, em Fortran usando GENCAN para a resolução dos subproblemas, e $\mathrm{AR}_{3}^{P}$, em Matlab ${ }^{\circledR}$ usando Gloptipoly para resolução dos subproblemas. Com isso, queremos ver se há alguma vantagem em se minimizar globalmente o modelo. Para base de comparação, rodamos todos os problemas com um método de Newton globalizado para minimização irrestrita. A estratégia de globalização adotada neste método consiste em computar a fatoração de Cholesky modificada da Hessiana, portanto, a cada iteração, resolvemos o sistema Newtoniano $\left(\nabla^{2} f\left(x^{k}\right)+D\right) d^{k}=-\nabla f\left(x^{k}\right)$, onde $D \in \mathbb{R}^{n \times n}$ é uma matriz diagonal tal que $\left(\nabla^{2} f\left(x^{k}\right)+D\right)$ seja definida positiva.

Os resultados são apresentados na Tabela 4.2. Nesta tabela, denotamos por $\mathrm{n}$ a quantidade de variáveis do problema, flag a saída do programa, $f\left(x^{*}\right)$ o valor da função objetivo no ponto ao qual convergiu o método, $\left\|g\left(x^{*}\right)\right\|_{\infty}$ a norma infinito do gradiente avaliado no ponto ao qual convergiu o método e \#it e \#f as quantidades de iterações e avaliações de função despendidas pelo método, respectivamente.

Para o método de Newton, flag zero significa que o método convergiu a um ponto aproximadamente estacionário do problema, isto é, a um ponto em que a norma do gradiente da função objetivo vale menos que $\epsilon$. Para o $\mathrm{AR}_{3}$, por sua vez, flag zero possui o mesmo significado que para Newton e flag -1 significa que o método empregado para minimização aproximada do modelo no Passo 2 do Algoritmo 2.2 não conseguiu convergir a um ponto $d^{k}$ tal que a condição (2.29) fosse satisfeita. Discorreremos acerca desta condição um pouco adiante ainda nesta seção.

Rodamos $\mathrm{AR}_{3}^{P}$ para problemas com $n$ até cinco pois, para valores de $n$ maior que cinco, Gloptipoly falhou ao tentar resolver um dos subproblemas. Tudo indica que isso foi causado pela sensibilidade numérica de Gloptipoly comentada ao final da Seção 4.1.2.

O principal resultado deste experimento foi constatar o desempenho superior do $\mathrm{AR}_{3}$ do ponto de vista de avaliações de função. No geral, pelo método de Newton, conforme a dimensão cresce, maior a quantidade de avaliações de função e maior a diferença para a quantidade consumida por $\mathrm{AR}_{3}$, 


\begin{tabular}{|c|c|c|c|c|c|c|c|c|c|c|c|c|c|c|}
\hline & \multicolumn{8}{|c|}{ NEWTON } & \multicolumn{6}{|c|}{$\mathrm{AR}_{3}^{G}$} \\
\hline$n$ & \multicolumn{3}{|c|}{$f\left(x^{*}\right)$} & \multicolumn{2}{|r|}{$\left\|g\left(x^{*}\right)\right\|_{\infty}$} & $\#$ it & \# & & flag & & $f\left(x^{*}\right)$ & $\mid g\left(x^{*}\right) \|_{\infty}$ & $\#$ it & $\# \mathrm{f}$ \\
\hline 1 & 0 & \multicolumn{2}{|l|}{$-3.29617855 \mathrm{D}-01$} & \multicolumn{2}{|c|}{$1.19459997 \mathrm{D}-13$} & 6 & 7 & 7 & 0 & \multicolumn{2}{|c|}{$-3.29617855 \mathrm{D}-01$} & $1.14042109 \mathrm{D}-12$ & 8 & 9 \\
\hline 2 & 0 & \multicolumn{2}{|l|}{$-2.18922777 \mathrm{D}+00$} & \multicolumn{2}{|c|}{$1.80012611 \mathrm{D}-09$} & 21 & 3 & & -1 & \multicolumn{2}{|c|}{$-2.18922777 \mathrm{D}+00$} & $6.53159107 \mathrm{D}-08$ & 13 & 14 \\
\hline 3 & 0 & \multicolumn{2}{|l|}{$6.11240967 \mathrm{D}-01$} & \multicolumn{2}{|c|}{$2.91778282 \mathrm{D}-09$} & 10 & 20 & & 0 & \multicolumn{2}{|c|}{$6.11240967 \mathrm{D}-01$} & $7.08347674 \mathrm{D}-09$ & 13 & 14 \\
\hline 4 & 0 & \multicolumn{2}{|l|}{$-1.04105410 \mathrm{D}+00$} & \multicolumn{2}{|c|}{$7.46880126 \mathrm{D}-12$} & 11 & 15 & & -1 & \multicolumn{2}{|c|}{$-1.04105410 \mathrm{D}+00$} & $1.27837354 \mathrm{D}-08$ & 14 & 15 \\
\hline 5 & 0 & \multicolumn{2}{|l|}{$-5.65321391 \mathrm{D}+00$} & \multicolumn{2}{|c|}{$8.16815477 \mathrm{D}-14$} & 7 & 10 & & 0 & \multicolumn{2}{|c|}{$-5.65321391 \mathrm{D}+00$} & $2.30926389 \mathrm{D}-14$ & 10 & 11 \\
\hline 10 & 0 & \multicolumn{2}{|l|}{$-1.19058407 \mathrm{D}-01$} & \multicolumn{2}{|c|}{$4.31940872 \mathrm{D}-10$} & 13 & 2 & & 0 & \multicolumn{2}{|c|}{$-1.19058407 \mathrm{D}-01$} & $9.02906902 \mathrm{D}-09$ & 18 & 19 \\
\hline 20 & 0 & \multicolumn{2}{|l|}{$-4.97222201 \mathrm{D}-01$} & \multicolumn{2}{|c|}{ 8.31433424D-09 } & 50 & 30 & & 0 & \multicolumn{2}{|c|}{$-4.97222201 \mathrm{D}-01$} & $3.85796795 \mathrm{D}-09$ & 26 & 27 \\
\hline 30 & 0 & \multicolumn{2}{|l|}{$-8.66485082 \mathrm{D}-01$} & \multicolumn{2}{|c|}{$3.59456646 \mathrm{D}-09$} & 37 & 145 & & 0 & \multicolumn{2}{|c|}{$-1.00448384 \mathrm{D}+00$} & $5.64059849 \mathrm{D}-09$ & 20 & 21 \\
\hline 40 & 0 & \multicolumn{2}{|l|}{$-5.21316143 \mathrm{D}-01$} & \multicolumn{2}{|c|}{$7.54819034 \mathrm{D}-09$} & 47 & $21 \%$ & & 0 & \multicolumn{2}{|c|}{$-6.54307076 \mathrm{D}-01$} & $4.57484304 \mathrm{D}-09$ & 26 & 27 \\
\hline 50 & 0 & $-6.97748237 \mathrm{D}-01$ & & 5.18467 & $241 \mathrm{D}-09$ & 84 & 68 & & 0 & -6.9774 & $8237 \mathrm{D}-01$ & $5.53374949 \mathrm{D}-09$ & 27 & 28 \\
\hline 100 & 0 & $-1.06684207 \mathrm{D}+00$ & & 4.12596 & $425 \mathrm{D}-09$ & 92 & 122 & & 0 & -2.0376 & $0912 \mathrm{D}+00$ & $5.91240733 \mathrm{D}-09$ & 37 & 38 \\
\hline & & & & & & & & $\mathrm{K}_{3}$ & & & & & & \\
\hline & & & $n$ & flag & & & & & & $\left(x^{*}\right) \|_{\infty}$ & $\#$ it & & & \\
\hline & & & 1 & -1 & -3.29617 & $55 \mathrm{D}-$ & & & 0785 & $4 \mathrm{D}-06$ & 10 & & & \\
\hline & & & 2 & -1 & -2.18922 & $77 \mathrm{D}-$ & & & $9727^{\prime}$ & $2 \mathrm{D}-05$ & 16 & & & \\
\hline & & & 3 & -1 & 6.11240 & $67 \mathrm{D}-$ & & & 0935 & $8 \mathrm{D}-06$ & 18 & & & \\
\hline & & & 4 & -1 & -1.04105 & $10 \mathrm{D}-$ & & & 4758 & $0 \mathrm{D}-07$ & 20 & & & \\
\hline & & & 5 & -1 & -5.65321 & $91 \mathrm{D}-$ & & & 6540 & $4 \mathrm{D}-06$ & 21 & & & \\
\hline
\end{tabular}

Tabela 4.2: Resultado dos experimentos com polinômios esparsos usando os métodos de Newton e $\mathrm{AR}_{3}$.

como ilustrado no gráfico da Figura 4.1 que relaciona a dimensão com a quantidade de avaliações de função.

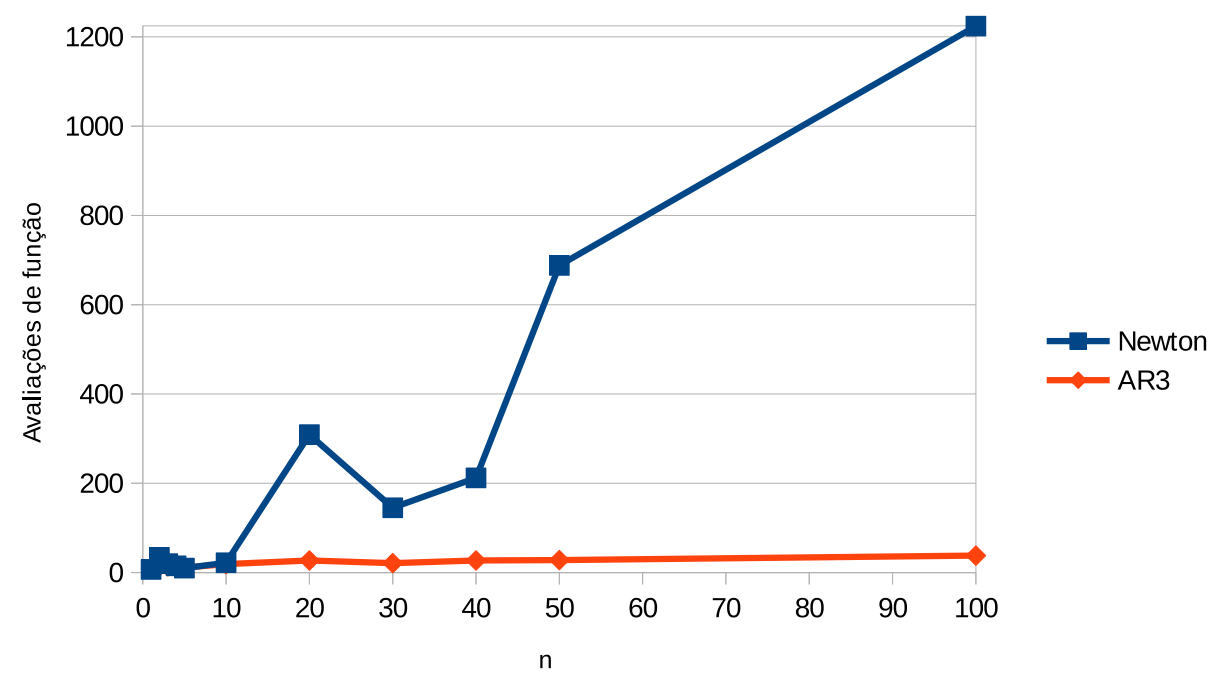

Figura 4.1: Avaliações de função versus dimensão do problema para os métodos de Newton e $\mathrm{AR}_{3}$.

Neste contexto, este resultado implica que $\mathrm{AR}_{3}$ será eficiente quando aplicado a problemas para os quais a avaliação da função objetivo $f$ do problema (2.1) custe muito mais que a avaliação do modelo $m_{p}$ definido em (2.18) para $p=3$. Neste caso, embora a minimização aproximada do modelo no Passo 2 do Algoritmo 2.2 consuma avaliações funcionais do modelo, ainda assim o custo seria inferior que avaliar muito mais vezes a função objetivo em métodos como Newton, considerado neste experimento.

Um aspecto que merece cuidado para o método $\mathrm{AR}_{3}$ é a minimização aproximada do modelo, em particular pela condição (2.29). Por natureza, métodos computacionais de otimização que se 
baseiam em atualização do ponto por passos, isto é, que tomam a cada iteração $x^{k+1}=x^{k}+d^{k}$, tendem a gerar passos $d^{k}$ muito pequenos (com relação à sua norma) quando se aproximam de uma solução do problema. Neste contexto, a condição (2.29) pode exigir uma norma muito pequena para o gradiente do modelo quando $\mathrm{AR}_{3}$ está se aproximando de uma solução do problema (2.1), dependendo do valor escolhido para a constante $\theta$. Neste contexto, consideremos a condição (2.29), no caso do $\mathrm{AR}_{3}$ dada por

$$
\left\|\nabla_{d} m_{3}\left(x^{k}, d^{k}, \sigma_{k}\right)\right\| \leq \theta\left\|d^{k}\right\|^{3} .
$$

Suponhamos que $\left\|d^{k}\right\|$ seja da ordem de $10^{-8}$. Por conseguinte, $\left\|d^{k}\right\|^{3}$ será da ordem de $10^{-24}$. Tomar $\theta=10^{16}$ implica que $\theta\left\|d^{k}\right\|^{3}$ será da ordem de $10^{-8}$, que, no geral, é uma condição razoável para norma do gradiente para métodos numéricos de otimização. Portanto, na prática, tomando $\theta=10^{16}$ estamos aceitando passos da ordem de $10^{-8}$ somente se o algoritmo para minimização de $m_{3}\left(x^{k}, d, \sigma_{k}\right)$ for bem sucedido na procura por um ponto aproximadamente estacionário deste problema. É importante destacar que nada impede que o algoritmo empregado para minimização do modelo falhe. Neste caso, como já dito anteriormente, nosso método para com flag -1.

Ainda se tratando de direções $d^{k}$ com norma muito pequena e do valor escolhido para a constante $\theta$, outro aspecto importante que tivemos que cuidar no método $\mathrm{AR}_{3}$ é a condição (2.30) para aceitação do ponto candidato. No caso em que $p=3$, pelo Lema 2.5, o denominador de $\rho$ calculado como em (2.30) é limitado inferiormente por algo que depende de $\left\|d^{k}\right\|^{4}$. Neste contexto, pode acontecer que, se $d^{k}$ possuir norma pequena, o denominador de $\rho$ seja numericamente nulo e que não seja possível avaliar a aceitação do novo ponto candidato. Neste caso, em nossa implementação, abordamos a execução do algoritmo, retornando o ponto corrente $x^{k}$ e flag -10 . Em nossos experimentos, não tivemos nenhum caso em que isso ocorreu; todavia, se tomássemos um valor para $\theta$ ainda maior que $10^{16}$, passos cuja norma fossem menores que $10^{-8}$ seriam aceitos e, neste caso, o cálculo de $\rho$ poderia falhar.

\subsection{Implementação computacional: $\mathrm{AR}_{2}^{s}$ e $\mathrm{AR}_{3}^{s}$}

Nesta seção, consideraremos o método proposto no Algoritmo 2.3 e descreveremos as estratégias tomadas para conceber uma versão implementável deste algoritmo.

Esta implementação parte do princípio, observado na seção anterior, que minimizar globalmente o modelo não é necessário nem vantajoso. Para isso, partimos do princípio que basta obter decréscimo simples no modelo (ou seja, apenas cumprir (2.45)) para obter bons passos. Este é o ponto de partida de nossa descrição na seção a seguir.

\subsubsection{Subproblemas}

A cada iteração do Algoritmo 2.3, calculamos $d^{k, j}$ no Passo 2 usando Gencan $[3,12,13]$ para resolver o problema

$$
\underset{d \in \mathbb{R}^{n}}{\operatorname{Minimizar}} m_{p}\left(x^{k}, d, \sigma_{k, j}\right)
$$

Nós implementamos as derivadas de ordem um e dois para $m_{p}$. Neste caso, Gencan reduz-se a um método de Newton globalizado. Para resolver o problema (4.44), rodamos Gencan partindo de $d^{0}=0$ e modificamos seu critério de parada para encerrar sua execução quando encontrar $d^{\ell}$ tal que

$$
\left\|\nabla_{d} m_{p}\left(x^{k}, d^{\ell}, \sigma_{k, j}\right)\right\| \leq \theta\left\|d^{\ell}\right\|^{p} .
$$


Vale dizer que um tal critério de parada foi pensado em particular para este caso, devido às exigências do Algoritmo 2.3 no Passo 2, que envolvem o iterando atual $d^{\ell}$, de Gencan, no lado direito da desigualdade (4.45).

Como Gencan produz uma sequência monotonicamente decrescente dos valores da função a ser minimizada, a condição (2.45) do Passo 2 do Algoritmo 2.2 vale naturalmente. Além disto, para problemas suaves e bem escalados, a condição (4.45) tende a ser mais permissiva nas primeiras iterações do Algoritmo 2.3 e mais rigorosa nas últimas iterações, quando $x^{k}$ estiver próximo a um minimizador local de $f$. Isto é uma propriedade interessante, visto que o esforço computacional será maior apenas nas últimas iterações do Algoritmo 2.3.

\subsubsection{Sobre as condições de descida e aceitação do ponto candidato}

Nós mostramos no Lema 2.10 que é sempre possível encontrar um $d^{k, j}$ que cumpre as condições (2.45) e (2.46) do Passo 2 do Algoritmo 2.3. Mais ainda, demonstramos que, para um valor suficientemente grande de $\sigma_{k, j},(2.48)$ vale. Todavia, na prática, o mecanismo não é tão simples e, por dificuldades numéricas, pode ser que os resultados teóricos não valham. Por isso, é importante lidar com essas situações com um devido cuidado.

Em primeiro lugar, qualquer software pode falhar em encontrar um ponto que cumpra (2.46) para uma tolerância muito pequena. Note que $\theta\left\|d^{k, j}\right\|^{p}$ pode tornar-se bem pequena quando $\left\|d^{k, j}\right\|$ for pequena, o que acontece com frequência quando, por exemplo, $x^{k}$ está próximo da solução do problema de minimizar $f(x)$.

Em segundo lugar, os valores suficientemente grandes de $\sigma_{k, j}$ que sempre cumprem (2.48), de acordo com o Corolário 2.11, dependem de $\alpha$, que é uma entrada do Algoritmo 2.3, e de $\xi_{1}$, que é uma constante desconhecida ligada ao problema. Por conseguinte, más escolhas para $\alpha$ e características intrínsecas do problema podem refletir negativamente no desempenho do algoritmo. Em outras palavras, o algoritmo pode descartar passos úteis que foram calculados usando um valor "bom" para $\sigma_{k, j}$ (bom no sentido de constituir um modelo $m_{p}$ que aproxima bem a função objetivo $f$ em torno do ponto corrente $x^{k, j}$ ) por não cumprir (2.48).

A fim de tratar esses dois problemas, iremos introduzir algumas condições de salvaguarda que não afetam a complexidade de pior caso do Algoritmo 2.3.

Para começarmos, assumamos que Gencan foi capaz de calcular uma direção $d^{k, j}$ tal que (2.46) valha. Neste caso, além de considerar a condição (2.48) como um critério de aceitação do ponto candidato, consideraremos também um critério de decréscimo fixo dado por

$$
f\left(x^{k}+d^{k, j}\right) \leq f\left(x^{k}\right)-\beta_{1} \epsilon^{\frac{p+1}{p}}
$$

para algum $\beta_{1}>0$ e $\epsilon$ a tolerância para a norma do gradiente tomada como critério de parada do algoritmo (veja (4.50) adiante). Note que a complexidade de pior caso do Algoritmo 2.3 ainda vale. De fato, por um lado, sempre que vale (2.46), $\left\|d^{k, j}\right\|$ é limitada inferiormente por uma constante não nula, para todo $k$ e todo $j$, como demonstramos no Lema 2.12 e, portanto, obtém-se o decréscimo $O\left(\epsilon^{(p+1) / p}\right)$ em $f$. Por outro lado, decréscimos da forma (4.46) naturalmente provocam o decréscimo $O\left(\epsilon^{(p+1) / p}\right)$ em $f$. Isso significa que a complexidade de pior caso ainda é a mesma. Na prática, espera-se que a condição (4.46) faça com que o algoritmo aceite passos "bons" que seriam rejeitados se apenas a condição (2.48) fosse considerada.

Por outro lado, consideremos que Gencan não foi capaz de calcular $d^{k, j}$ tal que valha (2.46). Neste caso, a teoria de complexidade de pior caso não vale mais, pois, como não vale (2.46), não vale a 
limitação inferior da direção no Lema $2.12 \mathrm{e}$, por conseguinte, não vale o resultado de complexidade estabelecido no Teorema 2.13. Neste caso, aceitaremos o ponto candidato se

$$
f\left(x^{k}+d^{k, j}\right) \leq f\left(x^{k}\right)-\beta_{2} \epsilon^{\frac{p+1}{p}},
$$

para algum $0<\beta_{2} \ll \beta_{1}$. Logo, aceitaremos um ponto que cause um decréscimo $O\left(\epsilon^{(p+1) / p}\right)$ na função objetivo. Com isso, o resultado de complexidade ainda vale. Note que tomamos $\beta_{2} \ll \beta_{1}$, com $\beta_{1}$ usado em (4.46), pois neste caso queremos ser mais permissivos que naquele caso. O raciocínio por trás desta ideia consiste no seguinte: se Gencan falhou em encontrar $d^{k, j}$ tal que vale (2.46), duas coisas podem ter acontecido: (i) o problema de minimizar $m_{p}$ é mal escalado ou (ii) a tolerância $\theta\left\|d^{k, j}\right\|^{p}$ é muito pequena. Em ambos os casos, ao invés de interromper a execução do algoritmo por causa de uma falha teórica, tentamos continuar o processo de otimização, aceitando o ponto que causa um pequeno decréscimo, fixo, em $f$.

Finalmente, suponha que não valem (2.46) nem (4.47). Neste caso, é necessário aumentar o parâmetro de regularização $\sigma$ para a próxima iteração. Entretanto se $\left\|d^{k, j}\right\|$ for muito pequena, há grandes chances de que o aumento no parâmetro de regularização $\sigma$ não cause impactos na iteração seguinte. Esse caso geralmente refere-se a uma estagnação do algoritmo, causada por dificuldades numéricas relacionadas a características intrínsecas do problema. Neste caso, não sabemos mais o que fazer e, portanto, interrompemos a execução do algoritmo.

\subsubsection{Norma do termo de regularização do modelo}

O modelo que desejamos minimizar é dado por

$$
m_{p}(x, d, \sigma)=T_{p}(x, d)+\frac{\sigma}{p+1}\|d\|^{p+1} .
$$

Consideremos o caso em que $p=3$. Nesta situação, a norma $\|d\|^{p+1}$ escolhida para o termo de regularização é relevante. Analisaremos duas possibilidades: usando norma 2 e norma 4.

Consideremos, inicialmente, o uso da norma 2. O modelo em questão, neste caso, é dado por

$$
m_{3}(x, d, \sigma)=T_{3}(x, d)+\frac{\sigma}{4}\|d\|_{2}^{4}
$$

Deste modo, o gradiente de $m_{3}$ é dado por

$$
\nabla_{d} m_{3}(x, d, \sigma)=\nabla_{d} T_{3}(x, d)+\left(\sigma\|d\|_{2}^{2}\right) d
$$

e a matriz Hessiana de $m_{3}$ é

$$
\nabla_{d}^{2} m_{3}(x, d, \sigma)=\nabla_{d}^{2} T_{3}(x, d)+(2 \sigma) d d^{T}+\left(\sigma\|d\|_{2}^{2}\right) I_{n},
$$

onde $I_{n}$ é a matriz identidade de ordem $n$.

Agora, consideremos o uso da norma 4. O modelo é dado por

$$
m_{3}(x, d, \sigma)=T_{3}(x, d)+\frac{\sigma}{4}\|d\|_{4}^{4}
$$

O gradiente de $m_{3}$ é dado por

$$
\nabla_{d} m_{3}(x, d, \sigma)=\nabla_{d} T_{3}(x, d)+\sigma \operatorname{diag}(d)^{3} \boldsymbol{e},
$$


onde $\operatorname{diag}(v)$ é uma matriz diagonal cujas entradas são os termos do vetor $v$ e $\boldsymbol{e}$ denota o vetor que possui 1 em suas componentes, de dimensão apropriada. A matriz Hessiana de $m_{3}$, neste caso, é dada por

$$
\nabla_{d}^{2} m_{3}(x, d, \sigma)=\nabla_{d}^{2} T_{3}(x, d)+3 \sigma \operatorname{diag}(d)^{2} .
$$

Notemos que a matriz (4.48) é densa, devido à matriz de posto $1, d d^{T}$, presente em sua fórmula. Não obstante, no caso em que a matriz Hessiana de $T_{3}$ for esparsa, o que depende, em grande parte, da matriz Hessiana da função objetivo, (4.49) também será esparsa. Por conseguinte, aplicar o algoritmo $\mathrm{AR}_{3}^{s}$ a problemas de grande porte usando norma 2 no termo regularizador não é uma escolha razoável.

\subsubsection{Implicações do uso de controle de passo}

Em métodos de regularização, o valor do parâmetro de regularização $\sigma$ determina quanto o modelo é uma boa aproximação para $f$ em torno de $x$. Todavia, não há um método intuitivo ou prático para se determinar o valor ideal de $\sigma$. Este valor está relacionado, geralmente, a características intrínsecas do problema, como escalamento, por exemplo.

Por conseguinte, muitas vezes no decorrer da otimização, pode acontecer que valores inapropriados para o parâmetro de regularização $\sigma$ sejam definidos. Consequentemente, a minimização de $m_{p}$ gerará uma direção $d^{k, j}$ que potencialmente não faça com que (2.48) valha.

Para ilustrar essa situação, consideremos o problema de minimizar a função de Rosenbrock dada por

$$
f(x)=100\left(y-x^{2}\right)^{2}+(1-x)^{2} .
$$

Consideremos uma execução do Algoritmo 2.3 com $p=3$, iniciando de $x^{0}=(-1,2 ; 1,0)^{T}$, com $\sigma_{0}=0$ e com os valores para as constantes $\sigma_{\min }=0,01, \gamma_{1}=0,5, \gamma_{2}=\gamma_{3}=10$ e $J=0$. Esta última determina que não há controle de passo. No ponto inicial, $f\left(x^{0}\right)=24,2$. A primeira iteração do algoritmo é bem sucedida, levando ao ponto $x^{1}=(-1,17,1,38)^{T}$, de forma que $f\left(x^{1}\right)=4,73$. Como o passo foi aceito, o valor de $\sigma$ se mantém, ou seja, $\sigma_{1}=\sigma_{0}=0$. Ilustramos esta iteração na Figura 4.2. Na segunda iteração, a direção calculada no Passo 2 leva a um valor de função 1411,85. Ilustramos este caso na Figura 4.3. A norma do passo dado nesta iteração é 4 vezes maior que a norma no ponto corrente. Neste caso, um controle de passo faria com que este passo fosse rejeitado sem sequer avaliar a função objetivo.

Este caso, e outros que surgem frequentemente na prática, nos levaram a incluir a estratégia de controle de passo no Algoritmo 2.3. Apenas reiterando, o principal objetivo é rejeitar direções que não causariam decréscimo na função objetivo, antes mesmo de avaliá-la.

\subsubsection{Critérios de parada}

Nós paramos a execução quando

1. o algoritmo convergiu para um ponto $\epsilon$-aproximadamente estacionário de primeira ordem, ou seja, quando o ponto corrente $x^{k}$ é tal que

$$
\left\|\nabla f\left(x^{k}\right)\right\|_{\infty} \leq \epsilon \stackrel{\text { def }}{=} \max \left\{\epsilon_{A}, \epsilon_{R}\left\|\nabla f\left(x^{0}\right)\right\|_{\infty}\right\}
$$

2. a quantidade de iterações excedeu um máximo de $k_{\max }$ iterações; 


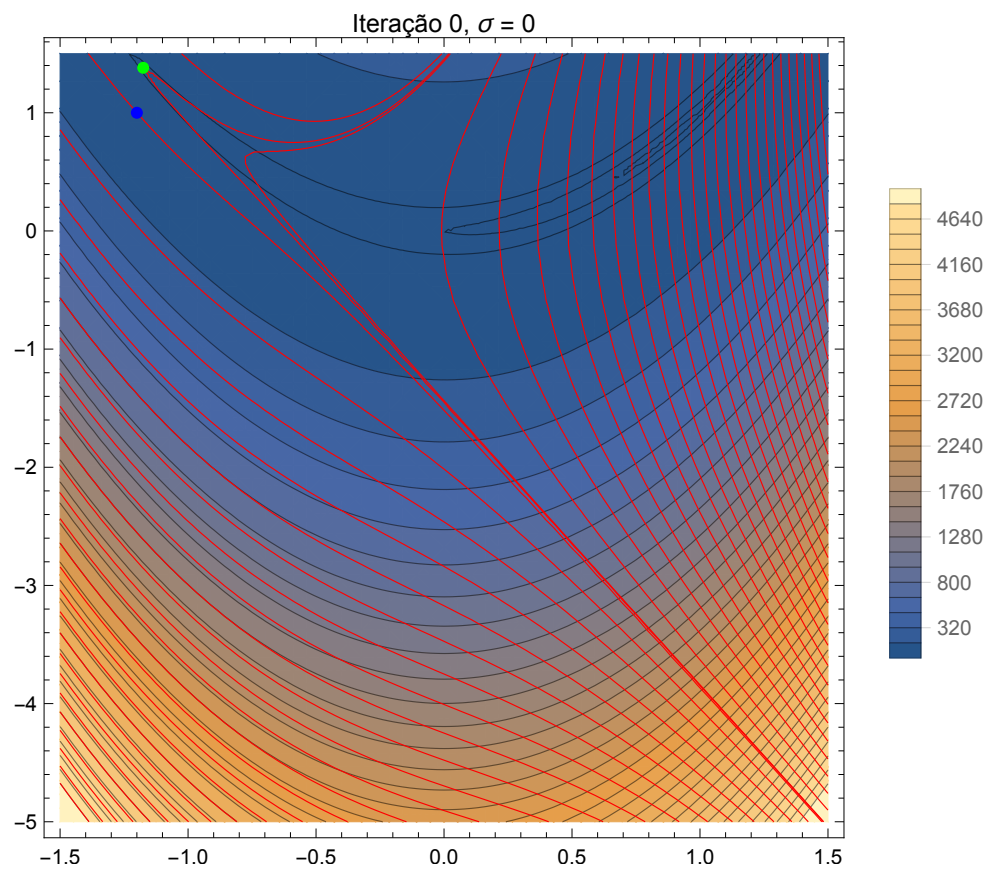

Figura 4.2: Primeira iteração de $\mathrm{AR}_{3}^{s}$.

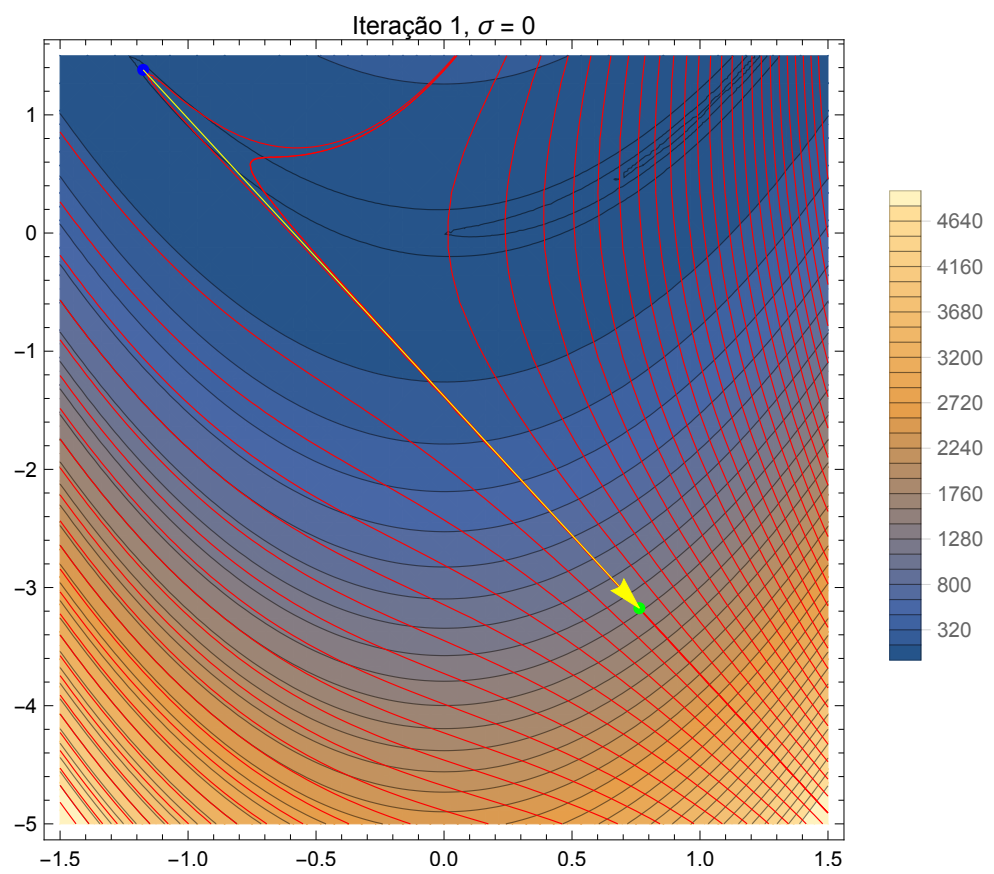

Figura 4.3: Segunda iteração de $\mathrm{AR}_{3}^{s}$, mal sucedida. 
3. a função objetivo parece ser ilimitada inferiormente, isto é,

$$
f\left(x^{k}\right) \leq f_{\min }
$$

4. Gencan convergiu a um ponto $x^{k, j}$ tal que não valem (2.46) e (4.47) e $\theta\left\|d^{k, j}\right\|^{p}$ é bem pequeno (pequeno significa algo em torno do zero da máquina, isto é, menor que ou igual a $10^{-16}$ ). Quando isso acontece, a teoria de complexidade não vale mais, e aumentar $\sigma_{k, j}$ na próxima iteração provavelmente não causará efeito, já que $\left\|d^{k, j}\right\|$ é pequena. Neste caso, o algoritmo poderia entrar em laço infinito e executar indefinidamente, aumentando sucessivamente $\sigma_{k, j}$. Este critério é acionado geralmente quando $\sigma_{k, j}$ é muito grande e $\left\|d^{k, j}\right\|$ é bem pequeno, e é indício de falta de progresso, como veremos nos experimentos numéricos na Seção 4.5.

\subsection{Experimentos numéricos}

Nós implementamos o Algoritmo 2.3 usando Fortran 2008, para $p=2$ e $p=3$, denotando-os por $\mathrm{AR}_{2}^{s}$ e $\mathrm{AR}_{3}^{s}$, respectivamente. Executamos os experimentos numéricos em um computador Intel ${ }^{\circledR}$ Core $^{\mathrm{TM}}$ i5 3.4 GHz e memória RAM DDR3 8 GB $1600 \mathrm{MHz}$, com sistema operacional macOS Sierra (versão 10.12.5). Compilamos os códigos usando Gfortran 6.3.0 com a opção -O3 habilitada.

\subsubsection{Experimentos com problemas clássicos da literatura}

Nesta seção, usaremos os 35 problemas-teste propostos por Moré, Garbow e Hillstrom [56]. Embora os autores tenham dividido os problemas em três conjuntos (minimização irrestrita, sistemas de equações não lineares e quadrados mínimos não lineares), nós consideramos todos os 35 problemas para compor nosso conjunto de testes. Para cada problema, são dadas expressões para $f_{i}(x)$, com $i=1, \ldots, m$, com $m \geq 1$. Nós consideramos o problema

$$
\underset{x \in \mathbb{R}^{n}}{\operatorname{Minimizar}} f(x)=\sum_{i=1}^{m} f_{i}^{2}(x) .
$$

Nosso objetivo consiste em investigar a usabilidade de métodos que utilizem derivadas terceiras. No caso, nossa implementação de $\mathrm{AR}_{3}^{s}$ utiliza-se de derivadas terceiras da função objetivo (4.51). O gradiente de (4.51) é dado por

$$
\nabla f(x)=2 \sum_{i=1}^{m} f_{i}(x) \nabla f_{i}(x) .
$$

A Hessiana, por sua vez, é dada por

$$
\nabla^{2} f(x)=2 \sum_{i=1}^{m}\left[\nabla f_{i}(x) \otimes \nabla f_{i}(x)+f_{i}(x) \nabla^{2} f_{i}(x)\right],
$$

onde $\otimes$ é uma operação definida entre dois tensores do $\mathbb{R}^{n}$, de ordens $a$ e $b$ respectivamente, e $C=A \otimes B$ é um tensor de ordem $a+b$ do $\mathbb{R}^{n}$ tal que

$$
C_{i_{1}, i_{2}, \ldots, i_{a+b}}=A_{i_{1}, i_{2}, \ldots, i_{a}} B_{i_{a+1}, i_{a+2}, \ldots, i_{a+b}} .
$$

A derivada terceira de (4.51), finalmente, é um tensor de ordem 3 do $\mathbb{R}^{n}$ dado por

$$
\nabla^{3} f(x)=2 \sum_{i=1}^{m}\left[\nabla^{2} f_{i}(x) \otimes \nabla f_{i}(x)+2 \nabla f_{i}(x) \otimes \nabla^{2} f_{i}(x)+f_{i} \nabla^{3} f_{i}(x)\right] .
$$


Portanto, o trabalho de desenvolvimento aqui consistiu em implementar as derivadas terceiras das $f_{i}, i=1, \ldots, m$, de todos os 35 problemas considerados, e compô-las da forma (4.52)-(4.54) para computar as derivadas da função objetivo.

Consideramos os seguintes valores para as constantes algorítmicas: $\alpha=10^{-8}, \eta_{1}=1000, \eta_{2}=3$, $\beta_{1}=10^{-4}, \beta_{2}=10^{4}, \sigma_{\min }=10^{-8}, \theta=100, \gamma_{1}=0.5, \gamma_{2}=10, \gamma_{3}=10$ e $J=20$. Para os critérios de parada, nós tomamos $\epsilon_{A}=10^{-8}, \epsilon_{R}=10^{-15}$ e $f_{\min }=-10^{8}$. À luz do que dissertamos na Seção 4.4, consideramos cinco variantes do Algoritmo 2.3:

$\mathbf{A R}_{2}^{s}$. Esta variante implementa o Algoritmo $2.3 \operatorname{com} p=2, J_{k}=J$ para todo $k$, e usa norma 2 no termo de regularização do modelo (2.18).

$\mathbf{A R}_{3}^{s}$. Esta variante implementa o Algoritmo $2.3 \operatorname{com} p=3, J_{k}=J$ para todo $k$, e usa a norma 4 no termo de regularização do modelo (2.18).

$\mathbf{A R}_{3}^{s}$ com norma 2. Esta variante implementa o Algoritmo $2.3 \operatorname{com} p=3, J_{k}=J$ para todo $k$ e usa norma 2 no termo de regularização do modelo (2.18).

$\mathbf{A R}_{3}^{s}$ com pré-processamento. Esta variante implementa o Algoritmo $2.3 \operatorname{com} p=3, J_{0}=J>0$ e $J_{k}=0$ para todo $k \geq 1$, e usa norma 4 no termo de regularização do modelo (2.18). Tal escolha para $J_{k}$ implica que o controle de passo é feito apenas na primeira iteração do algoritmo. Tal estratégia pode ser vista como um pré-processamento aplicado para calcular um parâmetro de regularização $\sigma$ inicial apropriado.

$\mathbf{A R}_{3}^{s}$ sem controle de passo. Esta variante implementa o Algoritmo $2.3 \operatorname{com} p=3, J_{k}=0$ para todo $k$, e usa norma 4 no termo de regularização do modelo (2.18). Esta escolha para $J_{k}$ implica que não faremos controle de passo no algoritmo.

Exibimos os resultados em forma de tabelas. Para cada tabela nesta seção, usamos os símbolos descritos na Tabela 4.3.

\begin{tabular}{ll}
\hline Símbolo & Significado \\
\hline Problema & número do problema [56] e sigla \\
$n$ & quantidade de variáveis \\
$m$ & quantidade de funções $f_{i}$ \\
flag & critério de Parada \\
$f\left(x^{\epsilon}\right)$ & valor da função no ponto final \\
$\left\|\nabla f\left(x^{\epsilon}\right)\right\|$ & norma do gradiente no ponto final \\
$\# f$ & quantidade de avaliações de função \\
$\# i t$ & quantidade de iterações \\
tempo & tempo de execução em segundos \\
\hline
\end{tabular}

Tabela 4.3: Significados dos símbolos nas Tabelas 4.5 e 4.6 .

Além disso, denotamos os critérios de paradas por indicadores numéricos (flags). Mostramos os significados destes indicadores na Tabela 4.4.

Vale mencionar que, pela definição do Algoritmo 2.3, a quantidade de avaliações das derivadas primeira, segunda e terceira da função objetivo é igual à quantidade de iterações acrescida de 1, por isso, para simplificar a exposição dos resultados, omitimos essas informações da tabela. 


\begin{aligned} & \hline flag Significado \\ & \hline 0 ponto estacionário de primeira ordem $x^{\epsilon}$ tal que $\left\|\nabla f\left(x^{\epsilon}\right)\right\|_{\infty} \leq \epsilon_{A} \\ & 1$ ponto estacionário de primeira ordem $x^{\epsilon}$ tal que $\left\|\nabla f\left(x^{\epsilon}\right)\right\|_{\infty} \leq \epsilon_{R}\left\|\nabla f\left(x^{0}\right)\right\|_{\infty} \\ &-3$ ponto final não cumpre $(2.46)$ nem $(4.47) \\ &$\hline\end{aligned}

Tabela 4.4: Significado dos indicadores de parada do algoritmo.

Nos experimentos a seguir, comparamos os métodos dois a dois com base em seus desempenhos nos 35 problemas. Sejam $f_{1}$ e $f_{2}$ os valores das funções objetivo nos pontos aos quais convergiram os dois métodos sendo comparados, e $f_{\text {best }}=\min \left\{f_{1}, f_{2}\right\}$. Como em [11], consideramos que ambos os métodos convergiram a soluções equivalentes se

$$
\frac{f_{i}-f_{\text {best }}}{\max \left\{1,\left|f_{\text {best }}\right|\right\}} \leq 10^{-4}, \text { para } i=1,2
$$

Em caso contrário, consideramos que os resultados não são comparáveis. Quando dizemos que "a versão A saiu-se melhor que a versão B", queremos dizer que são comparáveis e que a versão A consumiu menos avaliações de função que a versão B.

Nesta seção, nós queremos ilustrar com os experimentos (i) o uso da norma 2 e 4 no termo de regularização $\|s\|^{p+1}$ do modelo (2.18), (ii) o uso do controle de passo e (iii) a aplicação do algoritmo usando derivadas de até ordem dois e até ordem três.

Na Tabela 4.6, podemos observar os resultados dos experimentos comparando as variantes $\mathrm{AR}_{3}^{s}$ e $\mathrm{AR}_{3}^{s}$ com norma 2. $\mathrm{AR}_{3}^{s}$ com norma 2 consumiu menos avaliações de função em 11 problemas, enquanto $\mathrm{AR}_{3}^{s}$ também consumiu menos avaliações de função em outros 11 problemas. Houve 13 problemas em que ambos consumiram a mesma quantidade de avaliações de função. $\mathrm{AR}_{3}^{s} \mathrm{com}$ norma 2 consumiu $16.65 \%$ menos avaliações de função que $\mathrm{AR}_{3}^{s}$, em média. $\mathrm{AR}_{3}^{s}$, por sua vez, consumiu $11.64 \%$ menos avaliações de função que $\mathrm{AR}_{3}^{s}$ com norma 2, em média. Tais resultados nos levam a concluir que, em problemas de pequeno porte, os resultados são bem similares, a tal ponto que o uso de norma 2 ou norma 4 não afeta o desempenho geral do algoritmo em si, mas apenas em questões de memória e esparsidade, conforme discutimos na Seção 4.4.3. Na Seção 4.5.2, mostraremos um exemplo de um problema de grande porte cuja resolução torna-se viável apenas com o uso do modelo com norma 4.

Escolhemos três contextos de experimentação numérica a partir da definição de $J_{k}$ : (i) quando $J_{k}=0$, para todo $k$, o que implica que o controle de passo no Passo 3 do Algoritmo 2.3 não é aplicado, (ii) $J_{0}=J>0$ e $J_{k}=0$ para todo $k \geq 1$, o que significa que o controle de passo será aplicado apenas na primeira iteração do Algoritmo 2.3 e, nesse caso, aplicamos um tipo de estratégia de pré-processamento que, começando de $\sigma_{-1}=0$, encontra um valor apropriado para $\sigma_{0,0}$ e (iii) $J_{k}=J>0$ para todo $k$, o que significa que a estratégia de controle de passo será aplicada em todas as iterações do Algoritmo 2.3. Os resultados comparativos entre essas três implementações podem ser vistos na Tabela 4.6, comparando-se as versões $\mathrm{AR}_{3}^{s}$ sem controle de passo, $\mathrm{AR}_{3}^{s}$ com pré-processamento e $\mathrm{AR}_{3}^{s}$, respectivamente.

Por um lado, comparando-se $\mathrm{AR}_{3}^{s}$ contra $\mathrm{AR}_{3}^{s}$ com pré-processamento, $\mathrm{AR}_{3}^{s}$ consumiu menos avaliações de função em 24 problemas, enquanto $\mathrm{AR}_{3}^{s}$ com pré-processamento consumiu menos avaliações de função em apenas um problema (4 BBS). Em 10 problemas, ambos os métodos consumiram a mesma quantidade de avaliações de função. $\mathrm{AR}_{3}^{s}$ consumiu $16.31 \%$ menos avaliações de função nos problemas em que saiu-se melhor que $\mathrm{AR}_{3}^{s}$ com pré-processamento, em média.

Por outro lado, comparando-se $\mathrm{AR}_{3}^{s}$ contra $\mathrm{AR}_{3}^{s}$ sem controle de passo, $\mathrm{AR}_{3}^{s}$ consumiu menos avali- 
ações de função em 23 problemas, enquanto $\mathrm{AR}_{3}^{s}$ sem controle de passo consumiu menos avaliações de função em apenas um problema (4 BBS). Essas variantes consumiram a mesma quantidade de avaliações de função em 10 problemas, e houve um problema (6 JSF) em que $\mathrm{AR}_{3}^{s}$ e $\mathrm{AR}_{3}^{s}$ sem controle de passo convergiram a pontos distintos. $\mathrm{AR}_{3}^{s}$ consumiu $35.59 \%$ menos avaliações de função nos problemas em que saiu-se melhor que $\mathrm{AR}_{3}^{s}$ sem controle de passo, em média.

Por conseguinte, podemos concluir que vale a pena empregar a estratégia de controle de passo em todas as iterações do algoritmo.

Do ponto de vista teórico, quando as derivadas de ordem três da função objetivo estão disponíveis, $\mathrm{AR}_{3}^{s}$ possui complexidade de avaliação de pior caso $O\left(\epsilon^{-4 / 3}\right)$, enquanto $\mathrm{AR}_{2}^{s}$ possui complexidade de avaliação de pior caso $O\left(\epsilon^{-3 / 2}\right)$. Isso significa que, se tomarmos uma sequência de $\epsilon$ tendendo a zero, $\mathrm{AR}_{3}^{s}$ se sairá melhor que $\mathrm{AR}_{2}^{s}$ conforme $\epsilon$ se aproxima de zero. Entretanto, para um valor pequeno de $\epsilon$, gostaríamos de ver esse desempenho superior de $\mathrm{AR}_{3}^{s}$ sobre $\mathrm{AR}_{2}^{s}$ em termos de avaliações de função. Sob esta ótica, olhemos para os resultados da Tabela 4.5 e para os dados de $\mathrm{AR}_{3}^{s}$ na Tabela 4.6.

Exibimos na Tabela 4.5 os resultados de $\mathrm{AR}_{2}^{s}$ com os 35 problemas de Moré, Garbow e Hillstrom [56]. Em comparação com os resultados de $\mathrm{AR}_{3}^{s}$ na Tabela 4.6, $\mathrm{AR}_{2}^{s}$ se saiu melhor que $\mathrm{AR}_{3}^{s}$ em 8 problemas, enquanto o contrário aconteceu em 21 problemas. Houve 21 empates e 2 problemas (11 GUL e $31 \mathrm{BRB}$ ) para os quais $\mathrm{AR}_{2}^{s}$ e $\mathrm{AR}_{3}^{s}$ convergiram para pontos finais distintos. $\mathrm{AR}_{3}^{s}$ consumiu $28.58 \%$ menos avaliações de função nos 21 problemas em que se saiu melhor que $\mathrm{AR}_{2}^{s}$, em média.

Para os 35 problemas, ambos $\mathrm{AR}_{2}^{s}$ e $\mathrm{AR}_{3}^{s}$ pararam com flag -3 em apenas um problema (10 MEY). Todavia, ambos convergiram para a solução apontada em [56] para este problema. Para os problemas 11 GUL e $31 \mathrm{BRB}$, para os quais $\mathrm{AR}_{2}^{s}$ e $\mathrm{AR}_{3}^{s}$ convergiram a soluções distintas, embora ambos tenham convergido a pontos aproximadamente estacionários, $\mathrm{AR}_{3}^{s}$ de fato convergiu à solução do problema 11 GUL reportada em [56], enquanto $\mathrm{AR}_{2}^{s}$ convergiu para a solução do problema 31 BRB reportada em [56]. Para os outros 32 problemas, $\mathrm{AR}_{2}^{s}$ e $\mathrm{AR}_{3}^{s}$ convergiram a soluções equivalentes.

Portanto, o uso de derivadas terceiras, para o conjunto de problemas testes considerado, mostrou-se válido do ponto de vista de quantidade de avaliações de função. Isso significa que em problemas onde o custo de avaliar a função objetivo seja alto e que derivadas de ordem 3 estejam disponíveis, vale a pena o emprego do $\mathrm{AR}_{3}^{s}$. 


\begin{tabular}{|c|c|c|c|c|c|c|c|c|}
\hline \multirow{2}{*}{ Problema } & \multirow{2}{*}{$n$} & \multirow{2}{*}{$m$} & \multicolumn{6}{|c|}{$\mathbf{A} \mathbf{R}_{2}^{s}$} \\
\hline & & & flag & $f\left(x^{\epsilon}\right)$ & $\left\|\nabla f\left(x^{\epsilon}\right)\right\|$ & $\# \mathrm{f}$ & $\#$ it & tempo \\
\hline $1 \mathrm{ROS}$ & 2 & 2 & 0 & $1.6106 \mathrm{D}-27$ & $1.4 \mathrm{D}-13$ & 30 & 23 & 0.70 \\
\hline $2 \mathrm{FRF}$ & 2 & 2 & 0 & $4.8984 \mathrm{D}+01$ & $1.7 \mathrm{D}-09$ & 8 & 7 & 0.30 \\
\hline $3 \mathrm{PBS}$ & 2 & 2 & 0 & $5.3889 \mathrm{D}-29$ & $8.0 \mathrm{D}-11$ & 133 & 107 & 2.50 \\
\hline $4 \mathrm{BBS}$ & 2 & 3 & 0 & $0.0000 \mathrm{D}+00$ & $0.0 \mathrm{D}+00$ & 44 & 43 & 1.10 \\
\hline $5 \mathrm{BEA}$ & 2 & 3 & 0 & $1.1037 \mathrm{D}-27$ & $1.5 \mathrm{D}-13$ & 15 & 14 & 0.30 \\
\hline 6 JSF & 2 & 10 & 0 & $1.2436 \mathrm{D}+02$ & $2.0 \mathrm{D}-12$ & 11 & 10 & 0.20 \\
\hline $7 \mathrm{HFV}$ & 3 & 3 & 0 & $1.4476 \mathrm{D}-20$ & $1.8 \mathrm{D}-09$ & 13 & 12 & 0.50 \\
\hline $8 \mathrm{BAR}$ & 3 & 15 & 0 & $8.2149 \mathrm{D}-03$ & $9.1 \mathrm{D}-15$ & 12 & 11 & 0.40 \\
\hline 9 GAU & 3 & 15 & 0 & $1.1280 \mathrm{D}-08$ & $3.1 \mathrm{D}-09$ & 3 & 2 & 0.10 \\
\hline $10 \mathrm{MEY}$ & 3 & 16 & -3 & $8.7946 \mathrm{D}+01$ & $9.6 \mathrm{D}-05$ & 324 & 238 & 6.10 \\
\hline 11 GUL & 3 & 10 & 0 & $3.8500 \mathrm{D}-02$ & $0.0 \mathrm{D}+00$ & 2 & 1 & 0.20 \\
\hline 12 BTD & 3 & 10 & 0 & $2.0815 \mathrm{D}-18$ & $8.7 \mathrm{D}-10$ & 28 & 26 & 0.60 \\
\hline $13 \mathrm{PSF}$ & 4 & 4 & 0 & $1.2832 \mathrm{D}-12$ & $4.3 \mathrm{D}-09$ & 22 & 21 & 0.40 \\
\hline 14 WOD & 4 & 6 & 0 & $3.3688 \mathrm{D}-29$ & $1.6 \mathrm{D}-13$ & 50 & 40 & 1.10 \\
\hline $15 \mathrm{KOF}$ & 4 & 11 & 0 & $3.0751 \mathrm{D}-04$ & $1.7 \mathrm{D}-10$ & 15 & 11 & 0.40 \\
\hline $16 \mathrm{BDF}$ & 4 & 20 & 0 & $8.5822 \mathrm{D}+04$ & $3.2 \mathrm{D}-10$ & 9 & 8 & 0.20 \\
\hline 17 OS1 & 5 & 33 & 0 & $5.4649 \mathrm{D}-05$ & $3.3 \mathrm{D}-09$ & 70 & 46 & 1.70 \\
\hline $18 \mathrm{BIG}$ & 6 & 13 & 0 & $1.9809 \mathrm{D}-12$ & $6.2 \mathrm{D}-09$ & 47 & 42 & 1.20 \\
\hline 19 OS2 & 11 & 65 & 0 & $4.0138 \mathrm{D}-02$ & $1.0 \mathrm{D}-11$ & 20 & 16 & 0.70 \\
\hline 20 WAT & 6 & 31 & 0 & $2.2877 \mathrm{D}-03$ & $6.3 \mathrm{D}-13$ & 13 & 12 & 0.30 \\
\hline 21 ERO & 10 & 10 & 0 & $2.6655 \mathrm{D}-28$ & $2.9 \mathrm{D}-14$ & 38 & 24 & 0.80 \\
\hline $22 \mathrm{EPO}$ & 12 & 12 & 0 & $2.5399 \mathrm{D}-12$ & $4.4 \mathrm{D}-09$ & 22 & 21 & 0.40 \\
\hline 23 PE1 & 4 & 5 & 0 & $2.2500 \mathrm{D}-05$ & $3.0 \mathrm{D}-09$ & 53 & 38 & 1.00 \\
\hline 24 PE2 & 4 & 8 & 0 & $9.3763 \mathrm{D}-06$ & $3.4 \mathrm{D}-09$ & 144 & 107 & 2.50 \\
\hline $25 \mathrm{VDF}$ & 10 & 12 & 0 & $1.7353 \mathrm{D}-26$ & $2.6 \mathrm{D}-12$ & 15 & 14 & 0.20 \\
\hline 26 TRI & 10 & 10 & 0 & $2.7951 \mathrm{D}-05$ & $3.3 \mathrm{D}-10$ & 32 & 18 & 0.60 \\
\hline $27 \mathrm{BAL}$ & 40 & 40 & 0 & $5.2870 \mathrm{D}-18$ & $1.6 \mathrm{D}-09$ & 6 & 5 & 0.20 \\
\hline $28 \mathrm{DSB}$ & 10 & 10 & 0 & $9.1442 \mathrm{D}-22$ & $8.0 \mathrm{D}-11$ & 5 & 4 & 0.10 \\
\hline 29 DSI & 10 & 10 & 0 & $2.0352 \mathrm{D}-27$ & $4.8 \mathrm{D}-14$ & 6 & 5 & 0.10 \\
\hline 30 BRT & 10 & 10 & 0 & $2.0013 \mathrm{D}-23$ & $2.3 \mathrm{D}-11$ & 7 & 6 & 0.20 \\
\hline $31 \mathrm{BRB}$ & 10 & 10 & 0 & $2.5240 \mathrm{D}-21$ & $4.7 \mathrm{D}-10$ & 9 & 8 & 0.20 \\
\hline $32 \mathrm{LFF}$ & 10 & 10 & 0 & $1.3805 \mathrm{D}-30$ & $1.1 \mathrm{D}-15$ & 2 & 1 & 0.00 \\
\hline 33 LF1 & 10 & 10 & 0 & $2.1429 \mathrm{D}+00$ & $4.4 \mathrm{D}-11$ & 2 & 1 & 0.00 \\
\hline $34 \mathrm{LFZ}$ & 10 & 10 & 0 & $3.6471 \mathrm{D}+00$ & $9.5 \mathrm{D}-13$ & 2 & 1 & 0.00 \\
\hline $35 \mathrm{CHE}$ & 8 & 8 & 0 & $3.5169 \mathrm{D}-03$ & $7.2 \mathrm{D}-09$ & 28 & 12 & 1.00 \\
\hline
\end{tabular}

Tabela 4.5: Resultados numéricos do Algoritmo 2.3 dos 35 problemas de [56] com $p=2$. 


\begin{tabular}{|c|c|c|c|c|c|c|c|c|c|c|c|c|c|c|c|c|c|c|c|c|c|c|c|c|c|c|c|}
\hline \multirow{2}{*}{\multicolumn{2}{|c|}{ Problema }} & \multirow{2}{*}{$n$} & \multirow{2}{*}{$m$} & \multicolumn{6}{|c|}{$\mathbf{A R}_{3}^{s}$ com norma 2} & \multicolumn{6}{|c|}{$\mathbf{A R}_{3}^{s}$ sem controle de passo } & \multicolumn{6}{|c|}{$\mathbf{A R}_{3}^{s}$ com pré-processamento } & \multicolumn{6}{|c|}{$\mathbf{A R}_{3}^{s}$ (com norma 4 e controle de passo) } \\
\hline & & & & flag & $f\left(x^{\epsilon}\right)$ & $\left\|\nabla f\left(x^{\epsilon}\right)\right\|$ & $\# \mathrm{f}$ & $\#$ it & tempo & flag & $f\left(x^{\epsilon}\right)$ & $\left\|\nabla f\left(x^{\epsilon}\right)\right\|$ & $\# \mathrm{f}$ & $\#$ it & tempo & flag & $f\left(x^{\epsilon}\right)$ & $\left\|\nabla f\left(x^{\epsilon}\right)\right\|$ & $\# \mathrm{f}$ & $\#$ it & tempo & flag & $f\left(x^{\epsilon}\right)$ & $\left\|\nabla f\left(x^{\epsilon}\right)\right\|$ & $\# \mathrm{f}$ & $\#$ it & tempo \\
\hline 1 & ROS & 2 & 2 & 0 & $9,9660 \mathrm{D}-20$ & $8,4 \mathrm{D}-10$ & 16 & 13 & 0,50 & 0 & $2,4022 \mathrm{D}-26$ & $3,5 \mathrm{D}-13$ & 31 & 16 & 0,60 & 0 & $2,4022 \mathrm{D}-26$ & $3,5 \mathrm{D}-13$ & 31 & 16 & 0,60 & 0 & $2,4022 \mathrm{D}-26$ & $3,5 \mathrm{D}-13$ & 21 & 16 & 0,70 \\
\hline 2 & FRF & 2 & 2 & 0 & $4,8984 \mathrm{D}+01$ & $8,5 \mathrm{D}-14$ & 8 & 7 & 0,30 & 0 & $4,8984 \mathrm{D}+01$ & $8,5 \mathrm{D}-14$ & 14 & 6 & 0,30 & 0 & $4,8984 \mathrm{D}+01$ & $7,2 \mathrm{D}-10$ & 9 & 7 & 0,30 & 0 & $4,8984 \mathrm{D}+01$ & $7,2 \mathrm{D}-10$ & 8 & 7 & 0,30 \\
\hline 3 & PBS & 2 & 2 & 0 & $1,4845 \mathrm{D}-22$ & $4,7 \mathrm{D}-09$ & 116 & 92 & 2,70 & 0 & $1,4844 \mathrm{D}-22$ & $4,7 \mathrm{D}-09$ & 125 & 92 & 2,70 & 0 & $1,4844 \mathrm{D}-22$ & $4,7 \mathrm{D}-09$ & 117 & 92 & 2,60 & 0 & $1,4844 \mathrm{D}-22$ & $4,7 \mathrm{D}-09$ & 116 & 92 & 2,50 \\
\hline 4 & BBS & 2 & 3 & 0 & $0,0000 \mathrm{D}+00$ & $0,0 \mathrm{D}+00$ & 62 & 61 & 1,30 & 0 & $0,0000 \mathrm{D}+00$ & $0,0 \mathrm{D}+00$ & 15 & 13 & 0,30 & 0 & $0,0000 \mathrm{D}+00$ & $0,0 \mathrm{D}+00$ & 15 & 13 & 0,30 & 0 & $0,0000 \mathrm{D}+00$ & $0,0 \mathrm{D}+00$ & 70 & 69 & 1,40 \\
\hline 5 & BEA & 2 & 3 & 0 & $3,1586 \mathrm{D}-20$ & $6,8 \mathrm{D}-10$ & 10 & 9 & 0,40 & 0 & $0,0000 \mathrm{D}+00$ & $0,0 \mathrm{D}+00$ & 20 & 8 & 0,40 & 0 & $0,0000 \mathrm{D}+00$ & $0,0 \mathrm{D}+00$ & 10 & 8 & 0,40 & 0 & $0,0000 \mathrm{D}+00$ & $0,0 \mathrm{D}+00$ & 9 & 8 & 0,40 \\
\hline 6 & JSF & 2 & 10 & 0 & $1,2436 \mathrm{D}+02$ & $2,0 \mathrm{D}-12$ & 7 & 6 & 0,50 & 0 & $2,0200 \mathrm{D}+03$ & $0,0 \mathrm{D}+00$ & 15 & 1 & 0,30 & 0 & $1,2436 \mathrm{D}+02$ & $2,7 \mathrm{D}-11$ & 8 & 6 & 0,50 & 0 & $1,2436 \mathrm{D}+02$ & $2,7 \mathrm{D}-11$ & 7 & 6 & 0,50 \\
\hline 7 & $\mathrm{HFV}$ & 3 & 3 & 0 & $3,2105 \mathrm{D}-25$ & $3,0 \mathrm{D}-12$ & 9 & 8 & 0,50 & 0 & $2,0969 \mathrm{D}-24$ & $1,8 \mathrm{D}-11$ & 21 & 8 & 0,50 & 0 & $2,0969 \mathrm{D}-24$ & $1,8 \mathrm{D}-11$ & 10 & 8 & 0,40 & 0 & $2,0969 \mathrm{D}-24$ & $1,8 \mathrm{D}-11$ & 9 & 8 & 0,40 \\
\hline 8 & BAR & 3 & 15 & 0 & $8,2149 \mathrm{D}-03$ & $2,9 \mathrm{D}-09$ & 8 & 7 & 0,40 & 0 & $8,2149 \mathrm{D}-03$ & $1,7 \mathrm{D}-13$ & 19 & 7 & 0,40 & 0 & $8,2149 \mathrm{D}-03$ & $1,7 \mathrm{D}-13$ & 19 & 7 & 0,40 & 0 & $8,2149 \mathrm{D}-03$ & $1,7 \mathrm{D}-13$ & 8 & 7 & 0,40 \\
\hline 9 & GAU & 3 & 15 & 0 & $1,1280 \mathrm{D}-08$ & $8,3 \mathrm{D}-11$ & 2 & 1 & 0,10 & 0 & $1,1280 \mathrm{D}-08$ & $8,3 \mathrm{D}-11$ & 2 & 1 & 0,10 & 0 & $1,1280 \mathrm{D}-08$ & $8,3 \mathrm{D}-11$ & 2 & 1 & 0,10 & 0 & $1,1280 \mathrm{D}-08$ & $8,3 \mathrm{D}-11$ & 2 & 1 & 0,10 \\
\hline 10 & MEY & 3 & 16 & 1 & $8,7946 \mathrm{D}+01$ & $3,3 \mathrm{D}-05$ & 273 & 272 & 7,80 & -3 & $8,7946 \mathrm{D}+01$ & $5,0 \mathrm{D}-04$ & 970 & 827 & 17,20 & -3 & $8,7946 \mathrm{D}+01$ & $9,0 \mathrm{D}-04$ & 404 & 275 & 9,10 & -3 & $8,7946 \mathrm{D}+01$ & $9,0 \mathrm{D}-04$ & 331 & 275 & 9,50 \\
\hline 11 & GUL & 3 & 10 & 0 & $3,4242 \mathrm{D}-08$ & $7,5 \mathrm{D}-09$ & 104 & 84 & 2,00 & 0 & $2,4005 \mathrm{D}-08$ & $5,2 \mathrm{D}-09$ & 138 & 103 & 2,30 & 0 & $2,4005 \mathrm{D}-08$ & $5,2 \mathrm{D}-09$ & 132 & 103 & 2,50 & 0 & $2,4005 \mathrm{D}-08$ & $5,2 \mathrm{D}-09$ & 128 & 103 & 2,40 \\
\hline 12 & BTD & 3 & 10 & 0 & $4,4213 \mathrm{D}-19$ & $4,0 \mathrm{D}-10$ & 23 & 20 & 0,50 & 0 & $5,0016 \mathrm{D}-17$ & $4,2 \mathrm{D}-09$ & 34 & 22 & 0,60 & 0 & $5,0016 \mathrm{D}-17$ & $4,2 \mathrm{D}-09$ & 27 & 22 & 0,60 & 0 & $5,0016 \mathrm{D}-17$ & $4,2 \mathrm{D}-09$ & 26 & 22 & 0,60 \\
\hline 13 & PSF & 4 & 4 & 0 & $1,6309 \mathrm{D}-12$ & $7,0 \mathrm{D}-09$ & 20 & 19 & 0,60 & 0 & $3,8004 \mathrm{D}-13$ & $4,6 \mathrm{D}-09$ & 32 & 20 & 0,60 & 0 & $3,8004 \mathrm{D}-13$ & $4,6 \mathrm{D}-09$ & 32 & 20 & 0,60 & 0 & $3,8004 \mathrm{D}-13$ & $4,6 \mathrm{D}-09$ & 21 & 20 & 0,60 \\
\hline 14 & WOD & 4 & 6 & 0 & $4,9624 \mathrm{D}-30$ & $8,8 \mathrm{D}-14$ & 40 & 31 & 1,10 & 0 & $3,4123 \mathrm{D}-18$ & $5,3 \mathrm{D}-09$ & 49 & 30 & 1,00 & 0 & $3,4123 \mathrm{D}-18$ & $5,3 \mathrm{D}-09$ & 49 & 30 & 1,10 & 0 & $3,4123 \mathrm{D}-18$ & $5,3 \mathrm{D}-09$ & 38 & 30 & 1,00 \\
\hline 15 & $\mathrm{KOF}$ & 4 & 11 & 0 & $3,0751 \mathrm{D}-04$ & $3,5 \mathrm{D}-11$ & 19 & 17 & 0,50 & 0 & $3,0751 \mathrm{D}-04$ & $1,4 \mathrm{D}-11$ & 26 & 14 & 0,60 & 0 & $3,0751 \mathrm{D}-04$ & $1,4 \mathrm{D}-11$ & 26 & 14 & 0,50 & 0 & $3,0751 \mathrm{D}-04$ & $1,4 \mathrm{D}-11$ & 16 & 14 & 0,50 \\
\hline 16 & $\mathrm{BDF}$ & 4 & 20 & 0 & $8,5822 \mathrm{D}+04$ & $2,9 \mathrm{D}-11$ & 14 & 11 & 0,50 & 0 & $8,5822 \mathrm{D}+04$ & $6,7 \mathrm{D}-09$ & 25 & 11 & 0,50 & 0 & $8,5822 \mathrm{D}+04$ & $6,7 \mathrm{D}-09$ & 14 & 11 & 0,50 & 0 & $8,5822 \mathrm{D}+04$ & $6,7 \mathrm{D}-09$ & 13 & 11 & 0,50 \\
\hline 17 & OS1 & 5 & 33 & 0 & $5,4649 \mathrm{D}-05$ & $1,4 \mathrm{D}-10$ & 57 & 51 & 1,90 & 0 & $5,4649 \mathrm{D}-05$ & $3,0 \mathrm{D}-09$ & 74 & 50 & 2,00 & 0 & $5,4649 \mathrm{D}-05$ & $3,0 \mathrm{D}-09$ & 57 & 50 & 2,00 & 0 & $5,4649 \mathrm{D}-05$ & $3,0 \mathrm{D}-09$ & 56 & 50 & 1,90 \\
\hline 18 & BIG & 6 & 13 & 0 & $7,3354 \mathrm{D}-15$ & $3,0 \mathrm{D}-10$ & 29 & 19 & 0,60 & 0 & $1,2672 \mathrm{D}-16$ & $1,7 \mathrm{D}-09$ & 34 & 22 & 0,70 & 0 & $1,2672 \mathrm{D}-16$ & $1,7 \mathrm{D}-09$ & 34 & 22 & 0,70 & 0 & $1,2672 \mathrm{D}-16$ & $1,7 \mathrm{D}-09$ & 34 & 22 & 0,70 \\
\hline 19 & OS2 & 11 & 65 & 0 & $4,0138 \mathrm{D}-02$ & $3,0 \mathrm{D}-10$ & 27 & 20 & 1,10 & 0 & $4,0138 \mathrm{D}-02$ & $3,3 \mathrm{D}-09$ & 34 & 20 & 1,10 & 0 & $4,0138 \mathrm{D}-02$ & $3,3 \mathrm{D}-09$ & 27 & 20 & 1,20 & 0 & $4,0138 \mathrm{D}-02$ & $3,3 \mathrm{D}-09$ & 26 & 20 & 1,10 \\
\hline 20 & WAT & 6 & 31 & 0 & $2,2877 \mathrm{D}-03$ & $8,9 \mathrm{D}-12$ & 27 & 24 & 0,90 & 0 & $2,2877 \mathrm{D}-03$ & $9,5 \mathrm{D}-09$ & 35 & 21 & 0,80 & 0 & $2,2877 \mathrm{D}-03$ & $9,5 \mathrm{D}-09$ & 35 & 21 & 0,80 & 0 & $2,2877 \mathrm{D}-03$ & $9,5 \mathrm{D}-09$ & 22 & 21 & 0,80 \\
\hline 21 & ERO & 10 & 10 & 0 & $5,1350 \mathrm{D}-26$ & $2,0 \mathrm{D}-13$ & 24 & 18 & 0,70 & 0 & $0,0000 \mathrm{D}+00$ & $0,0 \mathrm{D}+00$ & 36 & 19 & 0,70 & 0 & $0,0000 \mathrm{D}+00$ & $0,0 \mathrm{D}+00$ & 36 & 19 & 0,70 & 0 & $0,0000 \mathrm{D}+00$ & $0,0 \mathrm{D}+00$ & 26 & 19 & 0,70 \\
\hline 22 & EPO & 12 & 12 & 0 & $7,5958 \mathrm{D}-12$ & $6,5 \mathrm{D}-09$ & 23 & 19 & 0,70 & 0 & $2,1647 \mathrm{D}-12$ & $2,3 \mathrm{D}-09$ & 33 & 20 & 0,80 & 0 & $2,1647 \mathrm{D}-12$ & $2,3 \mathrm{D}-09$ & 33 & 20 & 0,80 & 0 & $2,1647 \mathrm{D}-12$ & $2,3 \mathrm{D}-09$ & 25 & 20 & 0,80 \\
\hline 23 & PE1 & 4 & 5 & 0 & $2,2500 \mathrm{D}-05$ & $2,9 \mathrm{D}-10$ & 40 & 25 & 0,80 & 0 & $2,2500 \mathrm{D}-05$ & $3,3 \mathrm{D}-11$ & 47 & 30 & 0,90 & 0 & $2,2500 \mathrm{D}-05$ & $3,3 \mathrm{D}-11$ & 47 & 30 & 0,90 & 0 & $2,2500 \mathrm{D}-05$ & $3,3 \mathrm{D}-11$ & 47 & 30 & 0,80 \\
\hline 24 & $\mathrm{PE} 2$ & 4 & 8 & 0 & $9,3763 \mathrm{D}-06$ & $9,1 \mathrm{D}-09$ & 152 & 116 & 3,80 & 0 & $9,3763 \mathrm{D}-06$ & $9,9 \mathrm{D}-09$ & 144 & 102 & 3,50 & 0 & $9,3763 \mathrm{D}-06$ & $9,9 \mathrm{D}-09$ & 144 & 102 & 3,90 & 0 & $9,3763 \mathrm{D}-06$ & $9,9 \mathrm{D}-09$ & 134 & 102 & 3,70 \\
\hline 25 & VDF & 10 & 12 & 0 & $0,0000 \mathrm{D}+00$ & $0,0 \mathrm{D}+00$ & 13 & 9 & 0,70 & 0 & $0,0000 \mathrm{D}+00$ & $0,0 \mathrm{D}+00$ & 42 & 23 & 1,30 & 0 & $0,0000 \mathrm{D}+00$ & $0,0 \mathrm{D}+00$ & 27 & 23 & 1,30 & 0 & $0,0000 \mathrm{D}+00$ & $0,0 \mathrm{D}+00$ & 26 & 23 & 1,30 \\
\hline 26 & TRI & 10 & 10 & 0 & $2,7951 \mathrm{D}-05$ & $1,4 \mathrm{D}-13$ & 11 & 7 & 0,60 & 0 & $2,7951 \mathrm{D}-05$ & $4,2 \mathrm{D}-12$ & 19 & 6 & 0,50 & 0 & $2,7951 \mathrm{D}-05$ & $4,2 \mathrm{D}-12$ & 11 & 6 & 0,50 & 0 & $2,7951 \mathrm{D}-05$ & $4,2 \mathrm{D}-12$ & 10 & 6 & 0,60 \\
\hline 27 & BAL & 40 & 40 & 0 & $1,8751 \mathrm{D}-24$ & $4,9 \mathrm{D}-13$ & 4 & 3 & 2,20 & 0 & $1,8751 \mathrm{D}-24$ & $4,9 \mathrm{D}-13$ & 4 & 3 & 2,50 & 0 & $1,8751 \mathrm{D}-24$ & $4,9 \mathrm{D}-13$ & 4 & 3 & 2,30 & 0 & $1,8751 \mathrm{D}-24$ & $4,9 \mathrm{D}-13$ & 4 & 3 & 2,30 \\
\hline 28 & DSB & 10 & 10 & 0 & $4,0128 \mathrm{D}-18$ & $5,6 \mathrm{D}-09$ & 3 & 2 & 0,10 & 0 & $4,0128 \mathrm{D}-18$ & $5,6 \mathrm{D}-09$ & 3 & 2 & 0,10 & 0 & $4,0128 \mathrm{D}-18$ & $5,6 \mathrm{D}-09$ & 3 & 2 & 0,10 & 0 & $4,0128 \mathrm{D}-18$ & $5,6 \mathrm{D}-09$ & 3 & 2 & 0,10 \\
\hline 29 & DSI & 10 & 10 & 0 & $5,7803 \mathrm{D}-29$ & $8,4 \mathrm{D}-15$ & 4 & 3 & 0,10 & 0 & $5,7803 \mathrm{D}-29$ & $8,4 \mathrm{D}-15$ & 4 & 3 & 0,10 & 0 & $5,7803 \mathrm{D}-29$ & $8,4 \mathrm{D}-15$ & 4 & 3 & 0,10 & 0 & $5,7803 \mathrm{D}-29$ & $8,4 \mathrm{D}-15$ & 4 & 3 & 0,10 \\
\hline 30 & $\mathrm{BRT}$ & 10 & 10 & 0 & $4,1237 \mathrm{D}-23$ & $2,6 \mathrm{D}-11$ & 5 & 4 & 0,10 & 0 & $4,1237 \mathrm{D}-23$ & $2,6 \mathrm{D}-11$ & 5 & 4 & 0,10 & 0 & $4,1237 \mathrm{D}-23$ & $2,6 \mathrm{D}-11$ & 5 & 4 & 0,10 & 0 & $4,1237 \mathrm{D}-23$ & $2,6 \mathrm{D}-11$ & 5 & 4 & 0,10 \\
\hline 31 & $\mathrm{BRB}$ & 10 & 10 & 0 & $2,6802 \mathrm{D}+00$ & $1,2 \mathrm{D}-11$ & 14 & 13 & 0,70 & 0 & $2,6802 \mathrm{D}+00$ & $3,0 \mathrm{D}-11$ & 22 & 8 & 0,60 & 0 & $2,6802 \mathrm{D}+00$ & $3,0 \mathrm{D}-11$ & 11 & 8 & 0,50 & 0 & $2,6802 \mathrm{D}+00$ & $3,0 \mathrm{D}-11$ & 10 & 8 & 0,50 \\
\hline 32 & $\mathrm{LFF}$ & 10 & 10 & 0 & $9,1705 \mathrm{D}-30$ & $2,3 \mathrm{D}-15$ & 2 & 1 & 0,10 & 0 & $9,1705 \mathrm{D}-30$ & $2,3 \mathrm{D}-15$ & 2 & 1 & 0,10 & 0 & $9,1705 \mathrm{D}-30$ & $2,3 \mathrm{D}-15$ & 2 & 1 & 0,10 & 0 & $9,1705 \mathrm{D}-30$ & $2,3 \mathrm{D}-15$ & 2 & 1 & 0,10 \\
\hline 33 & LF1 & 10 & 10 & 0 & $2,1429 \mathrm{D}+00$ & $1,6 \mathrm{D}-11$ & 2 & 1 & 0,00 & 0 & $2,1429 \mathrm{D}+00$ & $1,6 \mathrm{D}-11$ & 2 & 1 & 0,10 & 0 & $2,1429 \mathrm{D}+00$ & $1,6 \mathrm{D}-11$ & 2 & 1 & 0,10 & 0 & $2,1429 \mathrm{D}+00$ & $1,6 \mathrm{D}-11$ & 2 & 1 & 0,10 \\
\hline 34 & LFZ & 10 & 10 & 0 & $3,6471 \mathrm{D}+00$ & $2,2 \mathrm{D}-11$ & 2 & 1 & 0,00 & 0 & $3,6471 \mathrm{D}+00$ & $2,2 \mathrm{D}-11$ & 2 & 1 & 0,10 & 0 & $3,6471 \mathrm{D}+00$ & $2,2 \mathrm{D}-11$ & 2 & 1 & 0,00 & 0 & $3,6471 \mathrm{D}+00$ & $2,2 \mathrm{D}-11$ & 2 & 1 & 0,10 \\
\hline 35 & $\mathrm{CHE}$ & 8 & 8 & 0 & $3,5169 \mathrm{D}-03$ & $2,6 \mathrm{D}-12$ & 18 & 12 & 0,80 & 0 & $3,5169 \mathrm{D}-03$ & $7,8 \mathrm{D}-12$ & 26 & 10 & 0,70 & 0 & $3,5169 \mathrm{D}-03$ & $7,8 \mathrm{D}-12$ & 16 & 10 & 0,70 & 0 & $3,5169 \mathrm{D}-03$ & $7,8 \mathrm{D}-12$ & 15 & 10 & 0,70 \\
\hline
\end{tabular}

Tabela 4.6: Resultados numéricos dos experimentos com os 35 problemas de [56] com as quatro variantes do Algoritmo 2.3 com $p=3$. 


\subsubsection{Um problema de grande porte}

Nesta seção, consideraremos o problema de empacotar $N$ esferas idênticas de raio $r$, centradas nos pontos $c_{1}, c_{2}, \ldots, c_{N}$, com $c_{i} \in \mathbb{R}^{3}$ para $i=1,2, \ldots, N$, em uma esfera maior de raio $R$ centrada na origem. Esta é uma das formas de abordar o problema de empacotamento descrito, por exemplo, em [17].

Em suma, consideraremos o problema

$$
\underset{x \in \mathbb{R}^{3 N}}{\operatorname{Minimizar}} f(x),
$$

onde $x=\left(c_{1}, c_{2}, \ldots, c_{N}\right)^{T}$ e

$$
f(x)=\underbrace{\sum_{i=1}^{N} \sum_{j=i+1}^{N} \max \left\{0,(2 r)^{2}-\left\|c_{i}-c_{j}\right\|_{2}^{2}\right\}^{4}}_{\text {Sobreposição }}+\underbrace{\sum_{i=1}^{N} \max \left\{0,\left\|c_{i}\right\|_{2}^{2}-(R-r)^{2}\right\}^{4}}_{\text {Viabilidade }} .
$$

Esta função objetivo possui as derivadas primeira, segunda e terceira contínuas. Elas foram computadas para podermos aplicar o Algoritmo $2.3 \operatorname{com} p=3$.

A principal dificuldade deste tipo de problema é o tamanho do raio da esfera maior. A escolha de $R$ baseia-se no fato de que a fração ocupada da esfera grande pelas esferas menores de raios iguais é aproximadamente $30 \%$ do volume da esfera grande [14]. Isto significa que

$$
\frac{N\left(\pi r^{3}\right)}{\pi R^{3}} \approx 0.3 \Rightarrow R \approx r \sqrt[3]{\frac{N}{0.3}}
$$

Nós tomaremos como ponto inicial um ponto cujas bolas menores estejam dentro da bola maior, isto é, uma configuração inicial onde as esferas de raio $r$ são uniformemente geradas dentro do cuboide $[-R, R]^{3}$ e aquelas que não estiverem dentro da esfera de raio $R$ são descartadas. Com isto, o ponto inicial contribui apenas para o somatório de sobreposição de (4.55), entretanto o somatório referente à viabilidade é nulo. Note que, ao decorrer da otimização, o somatório de viabilidade pode deixar de ser nulo. Uma tal configuração inicial é mais adequada do ponto de vista numérico, no sentido de que o valor inicial de $f$ possuirá valor menor, em norma.

A função objetivo (4.55) possui custo de avaliação $O\left(N^{2}\right)$, que é bastante alto conforme $N$ cresce. Com o objetivo de obter um custo de avaliação linear, isto é, $O(N)$, existe uma estratégia que lida com o somatório de sobreposição de (4.55). Nós seguiremos a sugestão dada em [17, Seção 2.1] e calcularemos o termo $\max \left\{0,(2 r)^{2}-\left\|c_{i}-c_{j}\right\|_{2}^{2}\right\}^{4}$ apenas para esferas $i$ e $j$ que estejam próximas o suficiente entre si. De forma geral, a ideia é particionar o espaço em regiões quadradas de lado $2 r$ de forma a cobrir a esfera de raio $R$ inteira. Desta maneira, os itens cujo centro estiverem em regiões não adjacentes certamente não se sobrepõem e, portanto, sua parcela no somatório em (4.55) não precisa ser computada. Por conseguinte, a fim de avaliar este somatório, levaremos em conta apenas os pares de esferas cujos centros estejam na mesma região, ou estejam em regiões adjacentes. Como as esferas tendem a estar "espalhadas" dentro da esfera de raio $R$, para cada esfera de raio $r$ as esferas adjacentes tendem a ser um número constante e, portanto, a avaliação do somatório de sobreposição consumirá tempo $O(N)$.

Os resultados dos experimentos numéricos são exibidos na Tabela 4.8, enquanto os significados dos indicadores são exibidos na Tabela 4.7. Embora consuma tempo de processamento muito maior que Algencan, vale dizer que as iterações de $\mathrm{AR}_{3}^{s}$ são muito mais eficientes. Para a instância com maior dimensão, por exemplo, $\mathrm{AR}_{3}^{s}$ consumiu 144 iterações e 173 avaliações de função, enquanto Algencan consumiu 177 iterações e 1075 avaliações de função. Comparando-se uma taxa de eficácia dada pela quantidade de iterações por avaliação de função, em média, a eficácia dos passos computados por $\mathrm{AR}_{3}^{s}$ é 0,79 , enquanto que por Algencan é 0,16. 


\begin{tabular}{ll}
\hline Símbolo & Significado \\
\hline$N$ & quantidade de esferas menores, a serem empacotadas \\
$R$ & raio da esfera maior \\
$\# \mathrm{f}$ & quantidade de avaliações de função \\
$\# \mathrm{~g}$ & quantidade de avaliações da derivada de ordem 1 da função objetivo \\
$\# \mathrm{~h}$ & quantidade de avaliações da derivada de ordem 2 da função objetivo \\
$\# \mathrm{t}$ & quantidade de avaliações da derivada de ordem 3 da função objetivo \\
$\#$ it & quantidade de iterações \\
tempo & tempo de execução, medido em segundos \\
\hline
\end{tabular}

Tabela 4.7: Significado dos símbolos da Tabela 4.8.

\begin{tabular}{|c|c|c|c|c|c|c|c|c|c|c|c|}
\hline \multirow{2}{*}{$\mathrm{N}$} & \multirow{2}{*}{$\mathrm{R}$} & \multicolumn{5}{|c|}{$\mathrm{AR}_{3}^{s}$} & \multicolumn{5}{|c|}{ Algencan } \\
\hline & & $\# \mathrm{f} \quad \# \mathrm{~g}$ & $\# \mathrm{~h}$ & $\# \mathrm{t}$ & $\#$ it & Tempo & $\# \mathrm{f}$ & $\# \mathrm{~g}$ & $\# \mathrm{~h}$ & $\#$ it & Tempo \\
\hline 1.000 & 15,00 & 163120 & 120 & 120 & 119 & 26,09 & 623 & 164 & 109 & 109 & 1,63 \\
\hline 2.000 & 18,80 & 216161 & 161 & 161 & 160 & 82,40 & 684 & 175 & 118 & 118 & 3,58 \\
\hline 4.000 & 23,71 & 125100 & 100 & 100 & 99 & 99,48 & 1126 & 179 & 177 & 177 & 6,27 \\
\hline 6.000 & 27,14 & 144119 & 119 & 119 & 118 & 552,72 & 1347 & 198 & 196 & 196 & 11,22 \\
\hline 8.000 & 29,88 & 157133 & 133 & 133 & 132 & 243,24 & 852 & 133 & 131 & 131 & 12,57 \\
\hline 10.000 & 33,00 & 173145 & 145 & 145 & 144 & 998,99 & 1075 & 179 & 177 & 177 & 20,30 \\
\hline
\end{tabular}

Tabela 4.8: Resultados numéricos dos testes com problemas de empacotamento de grande porte. 


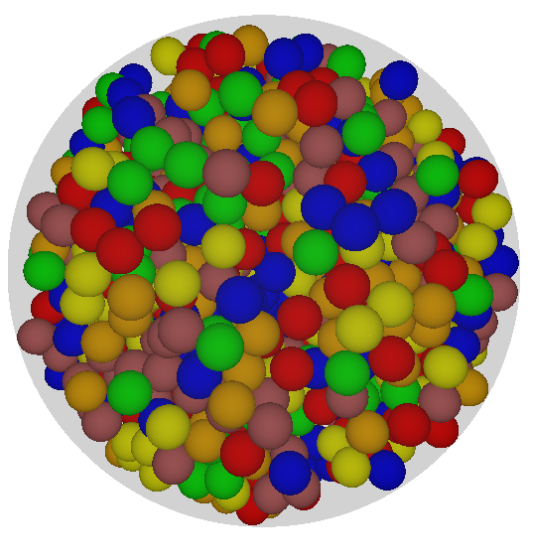

(a) 1.000 esferas

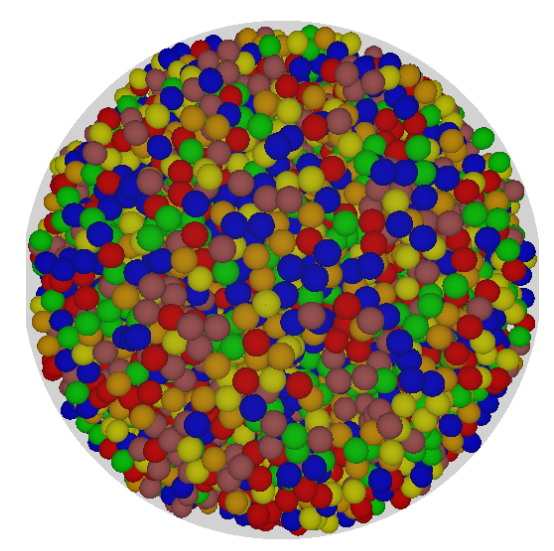

(d) 6.000 esferas

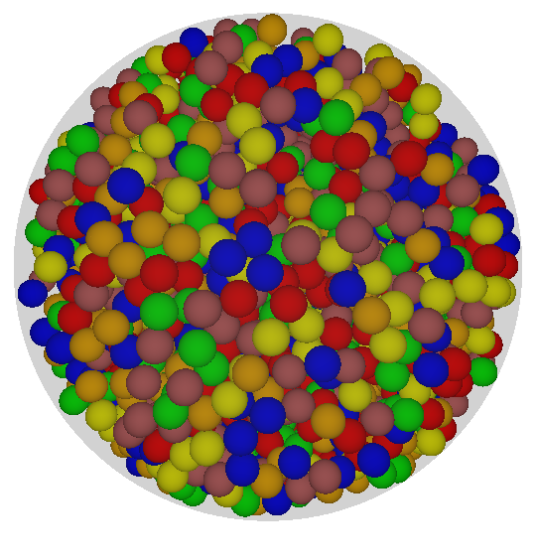

(b) 2.000 esferas

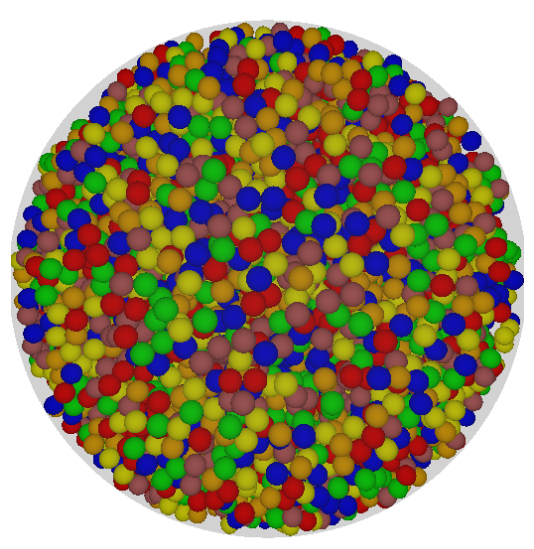

(e) 8.000 esferas

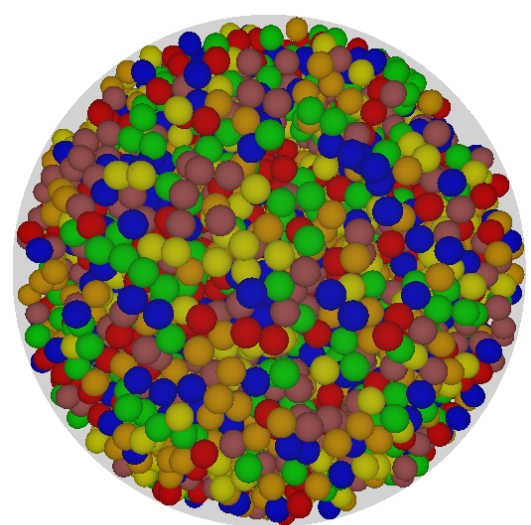

(c) 4.000 esferas

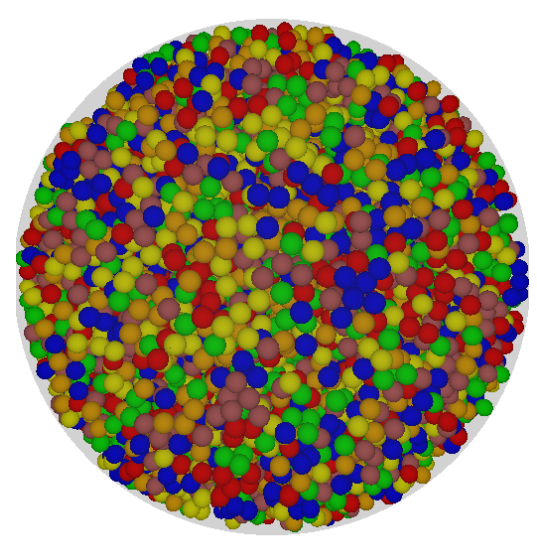

(f) 10.000 esferas

Figura 4.4: Resultados ilustrativos das soluções encontradas por $\mathrm{AR}_{3}^{s}$ para o problema de empacotamento. 


\section{Capítulo 5}

\section{Considerações finais e trabalhos futuros}

Nesta tese, estudamos complexidade de avaliação, no pior caso, para algoritmos de programação não linear. Neste contexto, propusemos três algoritmos: dois, similares, para problemas de minimização irrestrita e um para problemas de minimização com restrições, os três com análise de complexidade de avaliação de pior caso.

Para minimização sem restrições, propusemos dois algoritmos: o $\mathrm{AR}_{p}$ e o $\mathrm{AR}_{p}^{s}$. Ambos baseiam-se em modelos de ordem superior e métodos de regularização. Demonstramos, para ambos os métodos, que se as derivadas de ordem até $p$ da função objetivo estiverem disponíveis, a complexidade de avaliação de pior caso é $O\left(\epsilon^{-(p+1) / p}\right)$ para se obter convergência a um ponto aproximadamente estacionário com tolerância $\epsilon$. Estes foram os primeiros métodos que usam modelos de ordem superior a possuírem análise de complexidade.

Estudamos, ainda, a aplicabilidade dos algoritmos $\mathrm{AR}_{p}$ e $\mathrm{AR}_{p}^{s}$, pela implementação de ambos. Implementamos um protótipo de $\mathrm{AR}_{p}$ em Matlab ${ }^{\circledR}$ e uma versão mais consolidada de $\mathrm{AR}_{p}^{s}$ usando Fortran 2008. O objetivo principal, de ambas implementações, é aplicar métodos que usem derivadas terceiras da função objetivo, ou seja, o caso em que $p=3$. Os resultados dos experimentos mostram que $\mathrm{AR}_{3}^{s}$ é um método promissor, inclusive para problemas de grande porte. Em particular, este método mostra-se bem adequado para problemas em que o custo de avaliar a função objetivo seja alto. Trabalhos futuros, nesta linha, incluem propostas de implementação que melhorem o tempo de execução do algoritmo, que consiste em estudar novas maneiras para resolução dos subproblemas, e em encontrar problemas para os quais nosso método seja adequado. Neste contexto, já há pesquisas sendo desenvolvidas. Podemos citar, por exemplo, [5]. Publicamos os resultados teóricos do método $\mathrm{AR}_{p}$ em forma de artigo científico [10]. Dois outros artigos estão sendo preparados, um que trata do $\mathrm{AR}_{p}^{s}$, sua análise de complexidade, implementação e experimentação numérica [7] e outro que trata dos códigos atualizados para computação das funções, gradientes e Hessianas dos problemas de Moré, Garbow e Hillstrom [56], além de incorporar códigos para as derivadas terceiras [8].

Para minimização com restrições, propusemos um algoritmo do tipo target-following que chamamos de FTARGET. Este é o primeiro método para minimização com restrições que possui análise de complexidade e que converge a pontos KKT não escalados. Pode, potencialmente, utilizar-se de $\mathrm{AR}_{p} \mathrm{e}$ $\mathrm{AR}_{p}^{s}$ para resolução de subproblemas. Publicamos este algoritmo e sua análise de complexidade de avaliação na forma de artigo científico [9]. Trabalhos futuros acerca deste tema incluem, naturalmente, uma proposta de implementação prática deste método. Um trabalho relevante, nesta linha, encontra-se no relatório técnico de Curtis, Robinson e Samadi [36]. Este tópico será objeto de estudo do autor da presente tese em seu projeto de pós-doutorado. 


\section{Referências Bibliográficas}

[1] N. I. Akhiezer. The classical moment problem and some related questions in analysis. University mathematical monographs. Oliver \& Boyd, 1965.

[2] R. Andreani, J. M. Martínez, e B. F. Svaiter. A new sequential optimality condition for constrained optimization and algorithmic consequences. SIAM Journal on Optimization, 20(6):3533-3554, 2010 .

[3] M. Andretta, E. G. Birgin, e J. M. Martínez. Practical active-set Euclidian trust-region method with spectral projected gradients for bound-constrained minimization. Optimization, 54(3):305$325,2005$.

[4] C. Berg. The multidimensional moment problem and semigroups. Em Proceedings of Symposia in Applied Mathematics, volume 37, pp. 110-124. American Mathematical Society, 1987.

[5] E. Bergou, Y. Diouane, e S. Gratton. A line-search algorithm inspired by the adaptive cubic regularization framework, with a worst-case complexity $O\left(\epsilon^{-3 / 2}\right)$. Relatório técnico, 2017. Disponível em http://www.optimization-online.org/DB_FILE/2017/06/6083.pdf.

[6] E. Bergou, Y. Diouane, e S. Gratton. On the use of the energy norm in trust-region and adaptive cubic regularization subproblems. Relatório técnico, 2017. Disponível em http: //www.optimization-online.org/DB_HTML/2017/04/5980.html.

[7] E. G. Birgin, J. L. Gardenghi, J. M. Martínez, e S. A. Santos. On the use of third-order models with fourth-order regularization for unconstrained optimization. 2017. Submetido.

[8] E. G. Birgin, J. L. Gardenghi, J. M. Martínez, e S. A. Santos. Updated codes for Moré, Garbow and Hillstrom problems for unconstrained optimization software testing. Relatório técnico, 2017. Em preparação.

[9] E. G. Birgin, J. L. Gardenghi, J. M. Martínez, S. A. Santos, e Ph. L. Toint. Evaluation complexity for nonlinear constrained optimization using unscaled KKT conditions and high-order models. SIAM Journal on Optimization, 26(2):951-967, 2016.

[10] E. G. Birgin, J. L. Gardenghi, J. M. Martínez, S. A. Santos, e Ph. L. Toint. Worst-case evaluation complexity for unconstrained nonlinear optimization using high-order regularized models. Mathematical Programming, 163(1):359-368, 2017.

[11] E. G. Birgin e J. M. Gentil. Evaluating bound-constrained minimization software. Computational Optimization and Applications, 53(2):347-373, 2012.

[12] E. G. Birgin e J. M. Martínez. A box-constrained optimization algorithm with negative curvature directions and spectral projected gradients. Em Topics in Numerical Analysis: With Special Emphasis on Nonlinear Problems, pp. 49-60. Springer Vienna, Vienna, 2001.

[13] E. G. Birgin e J. M. Martínez. Large-scale active-set box-constrained optimization method with spectral projected gradients. Computational Optimization and Applications, 23(1):101-125, 2002. 
[14] E. G. Birgin e J. M. Martínez. Practical Augmented Lagrangian Methods for Constrained Optimization. SIAM, Philadelphia, 2014.

[15] E. G. Birgin e J. M. Martínez. On regularization and active-set methods with complexity for constrained optimization. Relatório técnico, 2017. Disponível em https://www.ime.usp.br/ egbirgin/publications/bmnuevogencan_siamformat.pdf.

[16] E. G. Birgin e J. M. Martínez. The use of quadratic regularization with a cubic descent condition for unconstrained optimization. SIAM Journal on Optimization, 27(2):1049-1074, 2017.

[17] E. G. Birgin e F. N. C. Sobral. Minimizing the object dimensions in circle and sphere packing problems. Computers $\&$ Operations Research, 35(7):2357-2375, 2008.

[18] J. Bolte, A. Daniilidis, O. Ley, e L. Mazet. Characterizations of Łojasiewicz inequalities: subgradient flows, talweg, convexity. Transactions of the Mathematical American Society, 362(6):3319$3363,2010$.

[19] N. Boumal, P.-A. Absil, e C. Cartis. Global rates of convergence for nonconvex optimization on manifolds. Relatório técnico, 2016. Disponível em https://arxiv.org/abs/1605.08101.

[20] C. Cartis, N. I. M. Gould, e Ph. L. Toint. On the complexity of steepest descent, Newton's and regularized Newton's methods for nonconvex unconstrained optimization problems. SIAM Journal on Optimization, 20(6):2833-2852, 2010.

[21] C. Cartis, N. I. M. Gould, e Ph. L. Toint. Adaptive cubic regularisation methods for unconstrained optimization. Part I: motivation, convergence and numerical results. Mathematical Programming, 127(2):245-295, 2011.

[22] C. Cartis, N. I. M. Gould, e Ph. L. Toint. Adaptive cubic regularisation methods for unconstrained optimization. Part II: worst-case function- and derivative-evaluation complexity. Mathematical Programming, 130(2):295-319, 2011.

[23] C. Cartis, N. I. M. Gould, e Ph. L. Toint. On the evaluation complexity of composite function minimization with applications to nonconvex nonlinear programming. SIAM Journal on Optimization, 21(4):1721-1739, 2011.

[24] C. Cartis, N. I. M. Gould, e Ph. L. Toint. An adaptive cubic regularization algorithm for nonconvex optimization with convex constraints and its function-evaluation complexity. IMA Journal of Numerical Analysis, 32(4):1662-1695, 2012.

[25] C. Cartis, N. I. M. Gould, e Ph. L. Toint. On the oracle complexity of first-order and derivativefree algorithms for smooth nonconvex minimization. SIAM Journal on Optimization, 22(1):66-86, 2012 .

[26] C. Cartis, N. I. M. Gould, e Ph. L. Toint. On the evaluation complexity of cubic regularization methods for potentially rank-deficient nonlinear least-squares problems and its relevance to constrained nonlinear optimization. SIAM Journal on Optimization, 23(3):1553-1574, 2013.

[27] C. Cartis, N. I. M. Gould, e Ph. L. Toint. On the complexity of finding first-order critical points in constrained nonlinear optimization. Mathematical Programming, 144(1-2):93-106, 2014.

[28] C. Cartis, N. I. M. Gould, e Ph. L. Toint. Corrigendum: On the complexity of finding first-order critical points in constrained nonlinear optimization. Mathematical Programming, pp. 1-16, 2016.

[29] C. Cartis, N. I. M. Gould, e Ph. L. Toint. Evaluation complexity bounds for smooth constrained nonlinear optimisation using scaled kkt conditions, high-order models and the criticality measure $\chi$. Relatório técnico, 2017. Disponível em https://arxiv.org/abs/1705. 04895. 
[30] C. Cartis, N. I. M. Gould, e Ph. L. Toint. Optimality of orders one to three and beyond: characterization and evaluation complexity in constrained nonconvex optimization. Relatório técnico, 2017. Disponível em https://arxiv.org/abs/1705.07285.

[31] C. Cartis, N. I. M. Gould, e Ph. L. Toint. Second-order optimality and beyond: characterization and evaluation complexity in convexly-constrained nonlinear optimization. Foundations of Computational Mathematics, 2017. No prelo.

[32] C. Cartis, Ph. R. Sampaio, e Ph. L. Toint. Worst-case evaluation complexity of non-monotone gradient-related algorithms for unconstrained optimization. Optimization, 64(5):1349-1361, 2015.

[33] C. Cartis e K. Scheinberg. Global convergence rate analysis of unconstrained optimization methods based on probabilistic models. Mathematical Programming, 2017. DOI: 10.1007/s10107-017-11374.

[34] X. Chen, Ph. L. Toint, e H. Wang. Partially separable convexly-constrained optimization with non-Lipschitzian singularities and its complexity. Relatório técnico, 2017. Disponível em https: //arxiv.org/abs/1704.06919.

[35] F. E. Curtis, D. P. Robinson, e M. Samadi. A trust region algorithm with a worst-case iteration complexity of $O\left(\epsilon^{-3 / 2}\right)$ for nonconvex optimization. Mathematical Programming, 162(1):1-32, 2017.

[36] F. E. Curtis, D. P. Robinson, e M. Samadi. Complexity analysis of a trust-funnel algorithm for equality constrained optimization. Relatório técnico, 2017. Disponível em http: //www.optimization-online.org/DB_FILE/2017/01/5793.pdf.

[37] J.-P. Dussault. $\mathrm{ARC}_{q}$ : a new adaptive regularization by cubics. Optimization Methods and Software, 2017. DOI: 10.1080/10556788.2017.1322080.

[38] B. Fuglede. The multidimensional moment problem. Expo. Math, 1(1):47-65, 1983.

[39] C. C. Gonzaga e E. W. Karas. Complexity of first-order methods for differentiable convex optimization. Pesquisa Operacional, 34:395-419, 2014.

[40] G. N. Grapiglia e Y. Nesterov. Regularized Newton methods for minimizing functions with Hölder continuous Hessians. SIAM Journal on Optimization, 27(1):478-506, 2017.

[41] G. N. Grapiglia, J. Yuan, e Y. Yuan. On the convergence and worst-case complexity of trustregion and regularization methods for unconstrained optimization. Mathematical Programming, 152(1):491-520, 2015.

[42] G. N. Grapiglia, J. Yuan, e Y. Yuan. A derivative-free trust-region algorithm for composite nonsmooth optimization. Computational and Applied Mathematics, 35(2):475-499, 2016.

[43] G. N. Grapiglia, J. Yuan, e Y. Yuan. Nonlinear stepsize control algorithms: Complexity bounds for first- and second-order optimality. Journal of Optimization Theory and Applications, 171(3):980997, 2016.

[44] S. Gratton, C. W. Royer, L. N. Vicente, e Z. Zhang. Direct search based on probabilistic feasible descent for bound and linearly constrained problems. Relatório técnico, 2017. Disponível em http://www.optimization-online.org/DB_FILE/2017/02/5867.pdf.

[45] G. Haeser, H. Liu, e Y. Ye. Optimality condition and complexity analysis for linearly-constrained optimization without differentiability on the boundary. Relatório técnico, 2017. Disponível em https://arxiv.org/abs/1702.04300.

[46] D. Henrion e J. B. Lasserre. GloptiPoly: Global Optimization over Polynomials with Matlab and SeDuMi. ACM Transactions on Mathematical Software, 29(2):165-194, 2003. 
[47] J. B. Lasserre. Global optimization with polynomials and the problem of moments. SIAM Journal on Optimization, 11(3):796-817, 2001.

[48] J. B. Lasserre. An explicit equivalent positive semidefinite program for nonlinear 0-1 programs. SIAM Journal on Optimization, 12(3):756-769, 2002.

[49] J. B. Lasserre. Polynomials nonnegative on a grid and discrete optimization. Transactions of the American Mathematical Society, 354:631-649, 2002.

[50] J. B. Lasserre. A semidefinite programming approach to the generalized problem of moments. Mathematical Programming, 112(1):65-92, 2008.

[51] S. Łojasiewicz. Ensembles semi-analytiques. Relatório técnico, Institut des Hautes Etudes Scientifiques, Bures-sur-Yvette, France, 1965. Disponível em http://perso.univrennes1.fr/michel.coste/Lojasiewicz.pdf.

[52] J. M. Martínez. On high-order model regularization for constrained optimization. Relatório técnico, 2017. Disponível em http://www.optimization-online.org/DB_FILE/2017/02/5850. pdf.

[53] J. M. Martínez e M. Raydan. Cubic-regularization counterpart of a variable-norm trust-region method for unconstrained minimization. Journal of Global Optimization, 68(2):367-385, 2017.

[54] J. M. Martínez e S. A. Santos. Métodos Computacionais de Otimização. Sociedade Brasileira de Matemática, Rio de Janeiro, 1995.

[55] R. D. C. Monteiro e B. F. Svaiter. On the complexity of the hybrid proximal extragradient method for the iterates and the ergodic mean. SIAM Journal on Optimization, 20(6):2755-2787, 2010.

[56] J. J. Moré, B. S. Garbow, e K. E. Hillstrom. Testing unconstrained optimization software. ACM Transactions on Mathematical Software, 7(1):17-41, 1981.

[57] A. S. Nemirovsky e D. B. Yudin. Problem Complexity and Method Efficiency in Optimization. Wiley-Interscience Series in Discrete Mathematics. John Wiley, New York, 1983.

[58] Y. Nesterov. Introductory Lectures on Convex Optimization. Kluwer Academic, Dordrecht, Países Baixos, 2004.

[59] Y. Nesterov e B. T. Polyak. Cubic regularization of Newton method and its global performance. Mathematical Programming, 108(1):177-205, 2006.

[60] L. Qi, Z. Wan, e Y. Yang. Global minimization of normal quartic polynomials based on global descent directions. SIAM Journal on Optimization, 15(1):275-302, 2004.

[61] R. T. Rockafellar. Lagrange multipliers and optimality. SIAM Review, 35(2):183-238, 1993.

[62] J. A. Shohat, J. D. Tamarkin, e A. M. Society. The Problem of Moments, volume 1 de Mathematical Surveys and Monographs. American Mathematical Society, 1943.

[63] L. N. Vicente. Worst case complexity of direct search. EURO Journal on Computational Optimization, 1(1):143-153, 2012.

[64] Nesterov Y. Gradient methods for minimizing composite objective function. Relatório técnico, Université Catholique de Louvain, Louvain, Bélgica, 2007. 